\title{
INTERNATIONAL UNION OF PURE AND APPLIED CHEMISTRY
}

\section{THEORY OF THE STRUCTURES OF NON-CRYSTALLINE SOLIDS}

\author{
Jozef Bicerano $^{1}$ and David Adler ${ }^{2}$ \\ ${ }^{1}$ Energy Conversion Devices, Inc., Troy, Michigan 48084, USA \\ Present address: Dow Chemical Company, Midland, Michigan 48674, USA \\ ${ }^{2}$ Department of Electrical Engineering and Computer Science, \\ Massachusetts Institute of Technology, Cambridge, Massachusetts 02139, USA \\ commissioned on the occassion of \\ INTERNATIONAL CONFERENCE ON THE THEORY OF THE \\ STRUCTURES OF NON-CRYSTALLINE SOLIDS \\ held in Bloomfield Hills, Michigan, USA: 3-6 June 1985
}




\title{
Theory of the structures of non-crystalline solids
}

\author{
Jozef Bicerano* and David Adler \\ Energy Conversion Devices, Inc., 1675 West Maple Road, Troy, Michigan 48084, USA \\ Department of Electrical Engineering and Computer Science, Massachusetts Institute \\ of Technology, Cambridge, Massachusetts 02139, USA
}

\begin{abstract}
The study of the structures and properties of non-crystalline solids and their interrelationships is a rapidly growing field of research, involving interdisciplinary efforts at the frontiers of chemistry, solid state physics, and materials science. These materials differ from the better-understood crystalline solids by the lack of long range three-dimensional translational periodicity in their atomic arrangements. Consequently, this study cannot make use of the simplification attendant with the use of repetitions of a small unit cell. We present a review of the state-of-the-art in the theoretical study of the structures of such non-crystalline solids. The topics covered include structural models, the nature of order and disorder, model systems of low dimensionality, chemical bonding considerations, thermodynamic and kinetic considerations, dynamic simulations, elastic and vibrational properties, electronic structure and properties, amorphous metals and alloys, silica glasses and related alloys, liquids and superlattices. It is our hope that this review will generate more interest among chemists in the scientific and technological problems and challenges presented by these materials.
\end{abstract}

\section{INTRODUCTION}

The atomic arrangement in non-crystalline (or "amorphous") solids lacks translational periodicity; therefore, unlike crystalline solids, these materials do not have a long range order consisting of the repetition in three dimensions of a well-defined unit cell. They have, for this reason, traditionally been in a no man's land between the realms of physics and chemistry. It has been impossible to describe their electronic wave-functions in terms of the powerful concepts and techniques of crystalline band theory (such as Brillouin zones, Bloch functions, $k$-space, etc.), discouraging solid state physicists from studying them. On the other hand, the fact that they are extended solids rather than molecules with a fixed number of atoms, has made their study appear to be outside the scope of conventional chemistry. Their lack of a repeating unit cell has also severely limited the detailed structural information that can be gained by such standard experimental techniques as $x$-ray diffraction. The apparent sensitive dependence of the structures of these materials, and hence of their physical, chemical, and electrical properties, on the methods used in preparation and processing, has further obscured the existing regularities.

During the past two decades, the study of the structures and properties of non-crystalline solids has gradually emerged as an exciting field of both experimental and theoretical research, involving interdisciplinary efforts at the frontiers of solid state physics, chemistry, and materials science. The barriers to communication between scientists from these diverse disciplines have been disappearing, as this field becomes better defined, with its own folklore and mythology, with its own dedicated workers and their characteristic technical jargon, and with the rapid progress brought about by three important motivating and enabling factors:

(1) The realization that non-crystalline solids have many important technological applications which go far beyond ordinary window glass. Some of these applications are: (i) as thin film photovoltaic materials used in solar cells for the conversion of light into electricity; (ii) in photographic films and xerographic drums; (iii) in electrical and optical switching; (iv) in electrical and optical data storage; ( $v$ ) in ultra-transparent optical fibers used for telecommunications; (vi) as hard protective coatings for tools and machine parts; (vii) as decorative coatings; ( $v i i i)$ as metallic glass ribbons used in transformer cores; and (ix) as active elements in large-area thin film displays such as flat-screen television sets. (In addition, polymeric materials, which ordinarily are also a type of non-crystalline solid, have many additional applications; however, these will not be considered in the present review.)

*Current Address: Dow Chemical Company, Central Research, Materials Science and Engineered Products Laboratory, 1702 Building, Midland, Michigan 48674, USA 
(2) The rapid progress in computer technology. The ability to process much larger amounts of data, and the much shorter times required to carry out large computations, are the cornerstones of this advance. Consequently, it has become increasingly more efficient to build large-scale structural models for non-crystalline solids via the use of computer programs, making it less necessary to go through the tedious process of building "ball-and stick" models; furthermore, it has become feasible to carry out large numerical calculations of electronic structure by using supercomputers. Finally, realistic simulations of dynamic processes such as glass formation via quenching, which require the use of techniques such as Monte Carlo and Molecular Dynamics, have become possible.

Several current directions in the advance of computer technology insure that computers will play an increasingly important part in future research on non-crystalline materials. For example, advances in parallel processing will probably be especially helpful in dynamic simulations where the collective behavior of large ensembles of particles is examined. The ability of smaller computers to perform larger calculations, and do this faster than ever before, will put sophisticated numerical techniques for electronic structure calculations within the reach of an increasing number of researchers. In this respect, it is especially encouraging to note the new classes of "supermini" and "advanced technology personal" computers. Finally, advances in three-dimensional color graphics technology, the declining cost of graphics hardware, and the increasing availability of graphics software, are all destined to help in computer-aided modeling and visualization of atomic arrangement patterns, just as they have helped in studying polymers and biological macromolecules.

(3) Recent advances in physics and chemistry. Some new theoretical approaches that have proved valuable in understanding amorphous solids include localization theory, percolation, the utilization of "curved space" techniques which were, in the past, mainly reserved for problems in general relativity and cosmology, and the use of higher and fractal dimensions, previously limited primarily to the analysis of unified field theories. On the chemical side, a gradually deepening understanding of the nature of chemical bonding has helped the study of non-crystalline solids by contributing to the determination of the local (or short range) order around the atoms. In the absence of long range periodic order, this local order is of paramount importance in understanding the structures and properties of these materials.

The theoretical progress has been paralleled and aided by advances both in the array and precision of experimental techniques available to us and in our understanding of how to apply these methods to non-crystalline solids and then extract structural information from the data. The techniques which have been useful in the latter regard include diffraction (x-ray, neutron and electron), EXAFS, various types of spectroscopy (Raman, IR, NMR, Mössbauer and ESR) and measurements of electrical and optical properties such as conductivity, luminescence and reflectivity.

The increased importance of research in non-crystalline solids has been highlighted by the awarding of Nobel Prizes to three scientists (N.F. Mott, P.W. Anderson and P.J. Flory) for their contributions to the field. The growth of interest in the area has also been reflected in a rapid increase in the number of conferences, symposia and workshops held on various aspects of the study of these materials. One of the most recent of these conferences was the International Conference on the Theory of the Structures of Non-Crystalline Solids, which was held 3-6 June 1985, at the Institute for Amorphous Studies in Bloomfield Hills, Michigan, U.S.A. The Proceedings of this Conference [1] are strongly recommended to readers who want to learn more about these materials. Two other recent books, one written by Zallen [2], and the other edited by Adler et al. [3] should also prove quite useful.

The present review includes a wide range of subjects. Structural models are discussed in Section II, focusing mainly on continuous random network and random close packing models. Several alternative models, and experimental techniques relevant to selecting and evaluating appropriate models, are also discussed. The subject of "order and disorder" is examined in Section III, in the contexts of short range order, "network momentum", icosahedral order, quasi-crystals and the curved space approach. Model systems of low dimensionality (with one or two dimensions), which facilitate the performance of certain calculations, are considered in Section IV. Chemical bonding considerations useful in understanding short range order are presented in Section $V$, while fundamental thermodynamic and kinetic considerations are discussed in Section VI. The use of dynamic (especially molecular dynamics) simulations is examined in Section VII. Section VIII is devoted to an examination of elastic and vibrational properties, and Section IX discusses the computation of the electronic structure and properties of nonmetallic amorphous solids. This discussion is followed by Section $X$ on amorphous metals and alloys, Section XI on silica glasses and related alloys, Section XII on liquids (which are structurally related to glasses), and Section XIII on superlattices. Our conclusions are summarized in Section XIV. In order to put the current developments into 
perspective, we also provide the general context and background, and present our own opinions and judgements concerning the work described, in each section, as well as making recommendations on how chemists can most easily enter the field and make significant contributions.

\section{STRUCTURAL MODELS}

\section{Standard models}

Unlike in crystalline solids, where the atomic-scale structure can be precisely determined, at least in principle, by diffraction experiments, in amorphous solids, the lack of a periodic repeating pattern makes it impossible to determine a unique structure. This may reflect a real ambiguity, since these materials could have a wide array of alternative structures with essentially the same total energy or it could represent simply our lack of knowledge of the precise structure. In any event, it therefore becomes essential to attempt to generate realistic models which will provide insights into the actual structures and properties of these materials.

The most developed homogeneous models of the atomic-scale structure of non-crystalline solids are $[2,4]$ :

1. The continuous random network (CRN) model, which has proved valuable in the understanding of covalent solids such as amorphous silicon (a-Si), silica (a-SiO $)$ and $\mathrm{a}-\mathrm{As}_{2} \mathrm{Se}_{3}$;

2. The random close packing (RCP) model, also sometimes referred to as the dense random packed (DRP) model, which has been quite useful in studying metallic glasses such as nickel-phosphorus, gold-silicon and copper-zirconium alloys;

3. The random coil model, which is primarily applicable to polymer-chain organic glasses such as polystyrene.

\section{CRN (continuous random network) models for covalent amorphous solids}

Two examples of the similarities and the differences $[2,4,5]$ between a CRN structure and its crystalline analog are presented in Figs. 1 and 2 . The following similarities can be seen between the pair of structures in each figure:

(a)

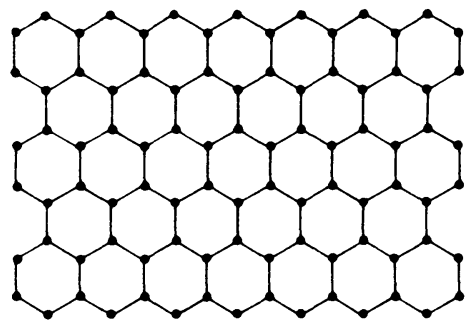

(b)

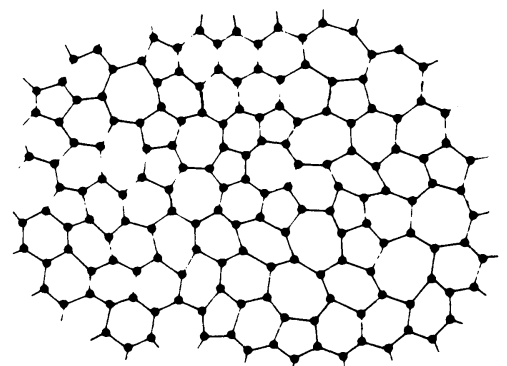

Fig. 1. (a) One layer of a three-fold coordinated crystalline solid, such as arsenic; (b) an analogous continuous random network (CRN) structure.

(a)

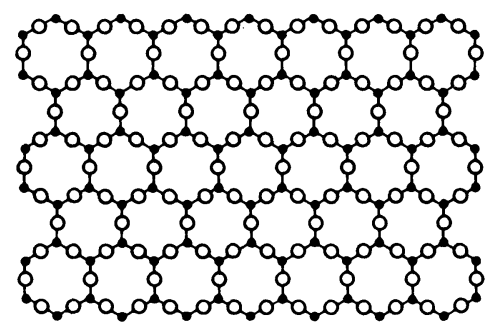

(b)

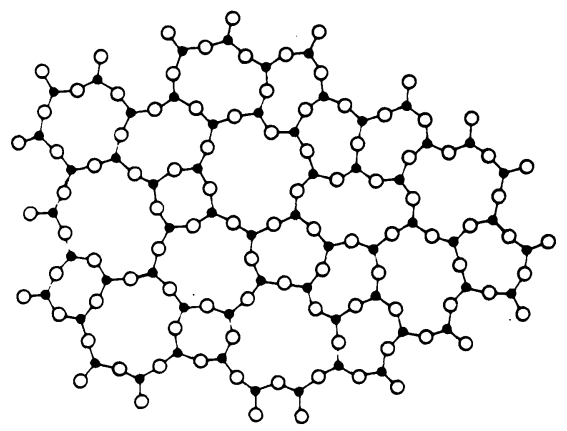

Fig. 2. (a) One layer of crystalline $\mathrm{As}_{2} \mathrm{~S}_{3}$ or $\mathrm{As}_{2} \mathrm{Se}_{3}$, where the large open circles represent $\mathrm{S}$ or $\mathrm{Se}$; (b) an analogous CRN structure. (Note that these two structures can be derived from the corresponding ones in Fig. 1 by replacing each bond between the three-fold coordinated atoms by a pair of bonds to an intervening two-fold coordinated "bridging" atom. This process is called a "decoration" transformation.) 
1. The coordination number of each atom is the same in both the CRN structure and its crystalline analog.

2. Bond lengths are nearly constant, because a great deal of energy is required to significantly increase or decrease bond lengths from their optimum values; in contrast, since it is considerably easier to bend bonds an thereby change the bond angles by several degrees than it is to stretch or compress bonds by several picometers, bond angle deviations are ubiquitous (see below).

3. Both pairs of structures are ideal in the sense that they contain no broken (or "dangling") bonds or other defect bonding configurations (i.e., atoms with non-optimal coordination), except at the surfaces where atoms may not be available to satisfy all the valence requirements.

There are two significant differences:

1. A significant spread in bond angles, not permitted in the crystal, is characteristic of the CRN structure. It is also worth noting, in Fig. 2, that there is more distortion of the bond angles around the divalent atoms than around the trivalent atoms, since distortions around the divalent atoms require less energy (they involve only one bond angle distortion, as opposed to two or three for trivalent atoms).

2. As a consequence of this spread in bond angles, long range order, i.e., translational periodicity, is absent from the CRN glass.

CRN-type models have been widely utilized in attempts to describe the overall structural features of many types of covalently bonded non-crystalline solids. These models have usually been built by actual construction of macroscopic ball-and-stick type physical representations of the assumed atomic-scale structures, followed by energetic relaxation via computer, using an assumed atom-atom pair potential. In order to generate a model which hopefully will represent the overall structural features reasonably well, models with a very large number of atoms have often had to be built, in spite of the tediousness of this task.

Some "classic" CRN models are: (1) the Polk [6], Polk and Boudreaux [7], Temkin, Paul and Connell [8], and Steinhardt, Alben and Weaire [9] models for tetrahedrally bonded amorphous semiconductors such as $\mathrm{a}-\mathrm{Si}$ and $\mathrm{a}-\mathrm{Ge}$; and (2) the Bell and Dean [10] model for a-SiO ${ }_{2}$, which is likely to also be useful for describing other amorphous $A_{2}$-type compounds with $(4,2)$ coordination such as $\mathrm{GeX}_{2}(\mathrm{X}=0, \mathrm{~S}, \mathrm{Se}$ or $\mathrm{Te})$, $\mathrm{SiTe}_{2}$, and $\mathrm{BeF}_{2}{ }^{\circ}$. Two recent examples of hand-built and computer-relaxed models are Popescu's models for a-Ge [1l] and for amorphous ice [12]. These types of models, which are in the spirit of the "classic" CRN models, are likely to continue to be built as long as adequate, fully computerized algorithms do not become readily available to workers in the field.

In recent years, considerable advances have been made in the fully computerized generation of realistic CRN models. Although the development of satisfactory algorithms is an extremely challenging task, the rewards of being able to automatically generate any number of such models without spending the time and effort needed to build each one of them atom by atom by hand, will certainly be very significant. The recent models of Wooten et al. [13] and of Lapiccirella et al. [14] are especially noteworthy. These have been built entirely by a systematic computer algorithm, and can be distinguished from hand-built models by the fact that they can be computer-relaxed to minimize the free energy by controlling the bond stretching and bond bending distortions.

The algorithm of Wooten et al. [13] enables the generation of realistic CRN structures with periodic boundary conditions. The resulting structures have no long range order, and contain 5-fold and 7-fold rings in addition to the 6-fold rings which are the only type of rings present in the crystalline (diamond) structure. On the other hand, short range order is preserved, i.e., the atoms remain fourfold coordinated with bond lengths and bond angles occupying narrow ranges around the crystalline bond length and tetrahedral bond angle respectively. The algorithm consists of two stages: (1) Starting from a large cluster with the diamond structure, long range order is destroyed and an amorphous structure is generated by several bond switching steps. (2) After an amorphous structure is obtained, further bond switches are performed to selectively lower the energy of the structure. At a given temperature $T$, a random topological rearrangement is accepted with a probability $p=1$ if the energy is reduced, and probability $p=\exp (-\Delta E / k T)$ if the energy is increased by $\Delta E$. This second stage might cause the amorphous structure to either revert to the initial diamond structure, which is the absolute minimum on the potential surface, or stabilize to become a truly metastable amorphous CRN, which is a relative minimum on the potential surface. The CRN structures obtained at the end of the algorithm give excellent agreement with the results of neutron diffraction experiments. Another noteworthy aspect of this work is its demonstration of how, as mentioned in Section I, advances in three-dimensional color graphics technology and molecular graphics software can aid in the visualization of atomic arrangement patterns in models of non-crystalline solids. 
The algorithm of Lapiccirella et al. [14], on the other hand, starts with a small, initially relaxed cluster, and adds atoms sequentially. Each new atom is added so as to saturate the dangling bond nearest to the center of mass of the cluster. After the addition of each atom, its position is examined relative to the other atoms in the cluster to prevent the inclusion of unrealistic bonding configurations such as bond lengths not within $10 \%$ of the normal bond length, multiple bonds, or 3-fold and 4-fold rings. After a few atoms are added in a way which satisfies these constraints, the structure is partially relaxed so as to avoid the generation of highly strained regions frozen in the bulk of the solid. When these steps are repeated to generate the full CRN, the structure obtained is both non-defective and spherical in shape. The spherical shape has the advantages of both minimizing the surface of the growing cluster and producing a model similar to that derived for an infinite number of atoms. In contrast to the algorithm of Wooten et al. [13] where all the atoms of the final CRN are present in the starting large cluster with the diamond structure, the algorithm of Lapiccirella et al. [14] can be visualized as consisting of the addition of successive concentric spherical shells to an original nucleus.

\section{Limitations of CRN models}

Although the CRN model has occupied a central position in the modeling of the structures of covalently bonded non-crystalline solids, it is by no means the only useful model. In fact, there is evidence that it may not be strictly valid for many amorphous alloys.

One obvious practical limitation of any type of model for an amorphous material is that, since there is no long range order and no repeating unit cell, any single structure, whether built by hand or by computer, is non-unique and only represents one example of an entire class of related conceivable structures. It has therefore been proposed [15] that the word "model" be used for a specific well-defined algorithm or set of rules for generating examples of such a related class of structures with any given number of atoms, rather than being used for individual examples. The overall properties of the model can then be determined by generating many examples by applying the algorithm and then averaging over these examples.

Idealized CRN models do not ordinarily admit any defect configurations, which must be introduced later. It is well-known from studies of crystalline solids, that defects and impurities, which are often present in concentrations of less than one per $10^{6}$ atoms, are crucial in determining important physical properties such as the magnitude of the electrical conductivity. Defects play a similar, very crucial role in non-crystalline solids, in which it is often much more difficult to pinpoint the specific types of defects involved and the roles they play [16]. It can be expected that they arise when the energy increase of a particular defect is comparable to that arising from the necessary bond distortions in their absence. Alternatively, relatively low-energy defects can arise for purely thermodynamic reasons [16]. In addition to dangling bonds, other types of point defects important in amorphous materials include: (1) Valence Alternation Pairs (VAP's) [16,17]. These low-energy defects are expected to be especially common in alloys which contain normally two-fold coordinated chalcogen atoms. The lowest energy electronic state of a chalcogen atom such as $\mathrm{S}$, Se or $\mathrm{Te}$ is a two-fold coordinated neutral one, denoted by $\mathrm{C}_{2}^{0}$. In general, however, it takes very little energy to break a bond and rearrange the resultant bonding pattern to replace two such atoms by a triply coordinated positively charged $\left(\mathrm{C}_{3}^{+}\right)$and a singly coordinated negatively charged $\left(C_{1}^{-}\right)$atom, in a reaction which might formally be represented as $2 C_{2}^{0} \rightarrow C_{3}^{+}+C_{1}^{-}$. Both $C_{3}^{+}$and $C_{1}^{-}$are low-energy defects since they have a filled outer octet of electrons, and no electrons in anti-bonding states. (2) Charge Transfer Defects (CTD's) $[16,18]$ in tetrahedrally bonded amorphous semiconductors such as a-Si and a-Ge, in which the lowest energy configuration is denoted $T_{4}^{0}$. These CTD's can be represented as a pair of positively and negatively charged atoms with one or two broken bonds $\left(\mathrm{T}_{3}^{+}+\mathrm{T}_{3}^{-}\right.$and $\mathrm{T}_{2}^{2+}+\mathrm{T}_{2}^{2-}$ respectively). These are not low-energy defects, but, as is discussed later, are expected to arise from strains upon processing.

A final limitation of CRN models, relevant to their application to alloys, is their assumption of chemical ordering, i.e., an inferred strong preference of atoms to bond more favorably with atoms of different kinds than with the same kind. For example, in $a-A_{2} \mathrm{Se}_{3}$, it is assumed that only As-Se bonds are present, although one would expect some As-As and Se-Se bonds to also be present as low-energy defect configurations, on simple thermodynamic grounds. In standard CRN models, homonuclear bonds are only allowed in non-stoichiometric alloys (e.g., in $\mathrm{As}_{1.9} \mathrm{Se}_{3.2}$ for which it is clearly impossible to find enough As atoms to satisfy the valence requirements of all the Se atoms). Because of all the limitations summarized in this subsection, CRN models should be viewed mainly as useful idealizations of the actual structure, and the possibility of significant deviations from them should be kept in mind whenever a new material is studied. 


\section{Alternative models for covalent amorphous solids}

The earliest structural model for covalent glasses was Lebedev's microcrystallite model [19] which assumed that the structure of glasses consisted of microcrystalline units. This model was challenged and eventually replaced by Zacharaisen's CRN model $[2,4,5]$. Not surprisingly, however, it is possible to find materials where neither the microcrystallite model nor the CRN model is completely adequate to represent the structure and bonding. Borate glasses are examples of such alloys. Extensive nuclear magnetic resonance (NMR) data [20] support the hypothesis that borate glasses such as $a-\mathrm{B}_{2} \mathrm{O}_{3}$ are not composed primarily of CRN's of individual $\mathrm{BO}_{4}$ and $\mathrm{BO}_{3}$ units. Instead, these small units form structural groupings such as boroxyl, diborate and metaborate units similar to the groupings that exist in the crystalline compounds of the particular borate system. Some of these structural groupings are illustrated in Fig. 3 [20]. These larger (but still quite small) units are then connected randomly to each other to form the glass structure. The situation is therefore intermediate between the microcrystallite and the CRN models. It has also been pointed out [21] that for many types of glass structures it is the intrinsically broken chemical order, i.e., deviations from perfect chemical ordering which are present even in stoichiometric alloys, that generates the defects wich control the electronic properties of the materials. This possible limitation of the Zacharaisen model can be seen from hyperfine effects, i.e., the proposed clustering into "molecular cluster networks" [22] of large morphologically and stoichiometrically distinct molecular clusters such as "chain" and "raft" configurations via molecular phase separation with intrinsically broken chemical order [21], and exciting recent Mössbauer [23], infrared (IR) [24], and Raman [24] experiments.

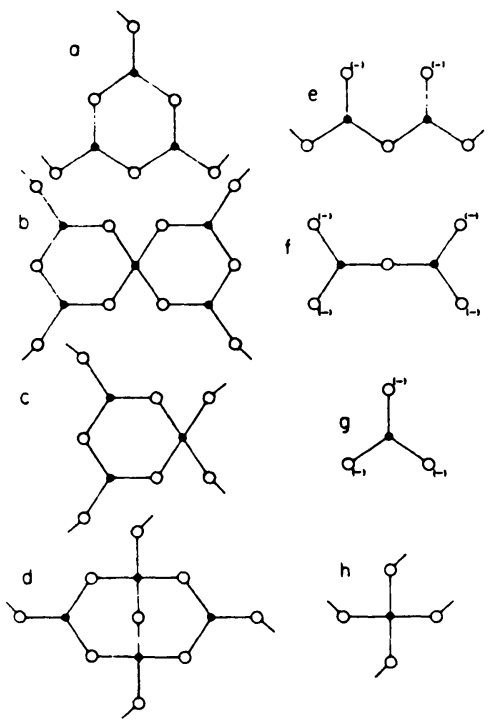

Fig. 3. Structural groupings in lithium borate glasses. Solid circles represent boron atoms, and open circles represent oxygen atoms. An open circle with a negative sign indicates a non-bridging oxygen. (a) Boroxol unit; (b) pentaborate unit; (c) triborate unit; (d) diborate unit; (e) metaborate unit; (f) pyroborate unit; (g) orthoborate unit; (h) loose $\mathrm{BO}_{4}$ unit. A tetraborate unit is formed by connecting one oxygen of the $\mathrm{BO}_{4}$ unit in the triborate unit to $\mathrm{a} \mathrm{BO}_{3}$ unit of the pentaborate unit [20].

These experiments are instructive since they probe the local environments around the atoms, rather than providing statistical averages over the bulk material. Their interpretation, however, is a matter of considerable controversy at the present time. As further Mössbauer, IR and Raman data become available and properly interpreted, a deeper understanding of the nature of the various types of glass structures is likely to emerge. After more than half a century, the field is still open for significant new conceptual advances!

\section{RCP (random close packed) models for metallic glasses}

The RCP (or alternatively DRP) models presently provide the most satisfactory representations for the structures of amorphous metals $[2,4]$. A traditional method for generating RCP type models is as follows [2]: A large number of hard spheres of uniform size are quickly poured and packed into a container having irregular surfaces, resulting in disordered configurations. The coordinates of the sphere centers are then determined by direct measurement. The overall long range interparticle attraction is usually provided by compression and gravity, while the short range repulsion is provided by the impenetrability of the balls used. Just like the CRN models for covalent amorphous solids, the resulting RCP structures are metastable, i.e., local minima on the free energy surface. Such models include those of Bernal [25], Scott and Kilgour [26], and Finney [27]. Fig. 4 [28] shows an example of a portion of an RCP structure.

Since atomic orientations in metallic glasses are much less directional than in covalently bonded solids, it is easier to write satisfactory computer algorithms to generate RCP structures than CRN structures. The earliest such computer program for the disordered packing of hard spheres was written by Bennett [29]. 


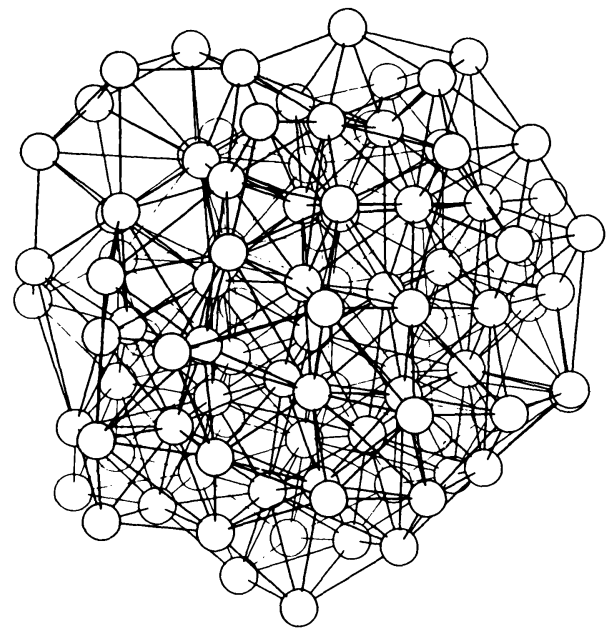

Fig. 4. Computer-generated portrait of a 100 -atom portion of a random close packed (RCP) structure [28].

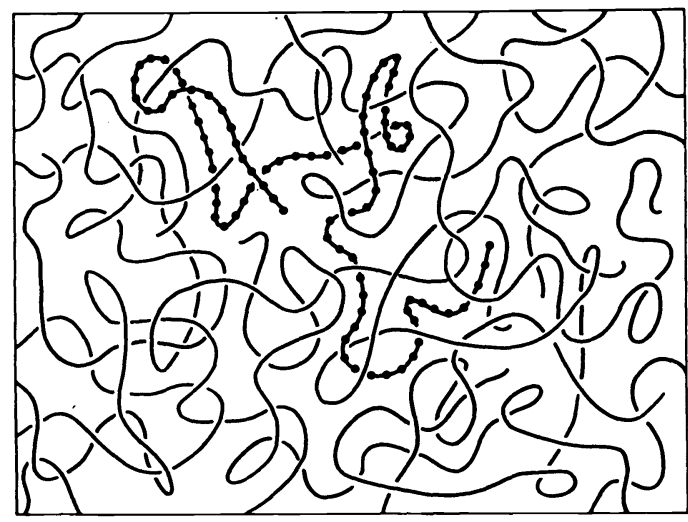

Fig. 6. Schematic sketch of the random coil model for polymeric glasses. One polymer chain has been "labeled" for ease of visualization [2].
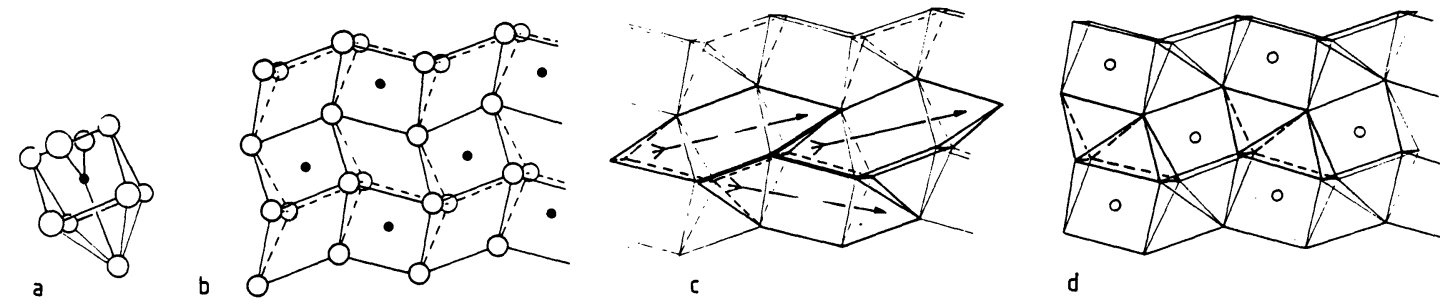

Fig. 5. (a) A boron atom (small filled circle) surrounded by six $\mathrm{Ni}$ atoms at the vertices of a trigonal prism and three further "capping" atoms forming half-octahedra (only one of which is shown). (b) The structure of $\mathrm{Ni}_{3} \mathrm{~B}$ showing a plane through the boron-centered trigonal prisms. Note the edge and vertex linkages. (c) Dissection of the structure into "arrowlike" units consisting of a trigonal prism "tipped" with a tetrahedron and with a half-octahedron as the "tail". (d) Dissection emphasizing the arrangement of tetrahedra and octahedra [30].

The RCP model for metallic glasses is clearly a direct analog of the CRN model for covalent amorphous solids. There is, however, evidence that the local environment of certain atoms in glassy metallic alloys is well-defined [30]. For example, the structures of transition metal/metalloid glasses such as $\mathrm{Ni}_{3} \mathrm{~B}$ seem to be be best represented by a 9-atom polyhedron centered on B, and likely to be a tricapped trigonal prism; therefore, the best structural models of such glasses may be generated not by random close packing of individtal atoms, but by random close packing of larger structural units such as tricapped trigonal prisms, tetrahedra and.octahedra. Fig. 5 [30] provides an illustration of these ideas, which are similar, in the context of metallic glasses, to Bray's observations [20] of deviations from the simple CRN model for the borate glasses. The lesson to be learned, again, is that the homogeneous models of the atomic-scale structure of non-crystalline solids should not be taken too literally, but should be viewed more as useful idealizations.

\section{Random coil model for polymeric glasses}

This model, sometimes called the spaghetti model, is due to Flory [31]. It can be viewed as an analog of the CRN and the RCP models, subject to the same types of limitations, and especially designed for organic polymeric glasses such as polystyrene. It is based on the random-walk configurations adopted by flexible, interpenetrating and intermeshed polymer coils. An example of a random coil model is sketched in Fig. $6[2,4]$.

\section{Conversion from RCP to CRN}

Raman studies [4] have been carried out on a-As $s_{3}$ thin films, prepared by solvent deposition from organic solution, in which the dissolved seed material originated from crystalline $\mathrm{As}_{2} \mathrm{~S}_{3}$, amorphous $\mathrm{As}_{2} \mathrm{~S}_{3}$, and crystalline $A s_{4} \mathrm{~S}_{4}$. Films derived from the two $A s_{2} \mathrm{~S}_{3}$ sources exhibit Raman spectra very similar to that of the bulk glass, suggesting a predominantly CRN type structure. On the other hand, the Raman spectra of films solvent-deposited from the $\mathrm{As}_{4} \mathrm{~S}_{4}$ source, as well as of vapor-deposited $\mathrm{a}-\mathrm{As}_{2} \mathrm{~S}_{3}$, can be best visualized as containing predominantly RCP character, with the presence of rather spherical molecular units of $\mathrm{As}_{4} \mathrm{~S}_{4}$ which are in a random close packing configuration. Upon annealing, these spectra gradually evolve in time to spectra much more similar to those in the bulk 


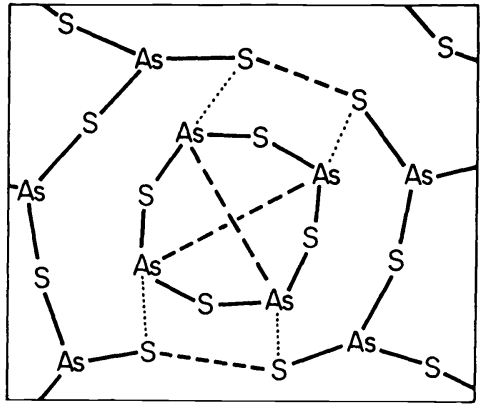

Fig. 7. A possible bond reconstruction mechanism for the digestion of a small molecule by the extended network of a chalcogenide glass. The bonds represented by the dotted lines replace the ones represented by the dashed lines [2].

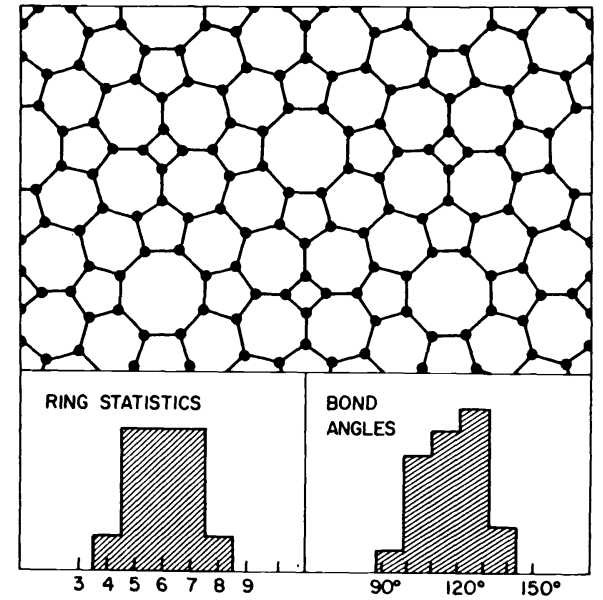

Fig. 8. An illustration of ring size and bond angle distributions [2].

glass, indicating that the structure is probably undergoing a phase transition from RCP to the more thermodynamically stable CRN by the "digestion" of the $\mathrm{As}_{4} \mathrm{~S}_{4}$ molecular units in a manner that is schematically represented in Fig. 7 [4]. This is a beautiful example of the interrelatedness of the various types of structural models, as well as of some of the subtleties involved (such as the significant effect of the method of preparation and processing on the type of structure that results).

\section{Comparisons with experiment}

We have already mentioned the Mössbauer [23], IR [24], and Raman [4,24] experiments performed to probe the local order, and the NMR experiments carried out on borate glasses [20]. The most familiar types of experiments used for analyzing the structures of non-crystalline solids, however, are neutron, electron, and x-ray diffraction [32], and controversies are quite common between proponents of Mössbauer and Raman experiments and those of diffraction experiments concerning which type of experiment is more informative. We feel that such controversies are unnecessary, since each type of experiment provides some useful information not available from any of the others. It seems more fruitful to attempt a synthesis of these results, with full awareness of the strengths and limitations of each method. Furthermore, most of these types of experiments are not too different from the techniques used by structural chemists in examining molecules, crystals, and biological macromolecules. We therefore strongly encourage experimental structural chemists looking for new challenges to consider getting involved in such experiments on amorphous solids.

Since amorphous solids lack periodicity, overall structural symmetry and long range order, there is no simple structural formalism (such as a unit cell repeating by translational symmetry) that can be used in the calculation of microscopic properties. The results of a diffraction experiment can, in some rare cases, be non-unique even for a crystalline solid, with the calculated set of vector distances corresponding to more than one possible set of Cartesian coordinates. For non-crystalline solids, in which these experiments can produce only a one-dimensional (scalar) correlation function (whereas the underlying structures are, of course, three-dimensional), the ambiguity is far more severe. Agreement of the predictions of a structural model with the diffraction data is therefore a necessary but not sufficient condition for the validity of a model. In other words, although any model which is inconsistent with carefully obtained diffraction data is certainly wrong and must be rejected, even a model which gives a perfect fit has no guarantee that it gives the correct structure, since other models may well exist that fit the data just as well [32].

Diffraction data from covalent non-crystalline solids are often divided into two classes: (1) short range order, involving the order within a single structural unit and its connection to its immediate neighbors [32], i.e., characterized by the coordination numbers of the atoms involved, and the bond lengths and bond angles between them [33]; and (2) intermediate (or medium) range order, describing the overall network topology, often characterized in terms of the "ring statistics", i.e., the distribution describing the frequency of occurrence of n-atom rings as a function of $n$. An illustration of ring statistics and bond angle distributions is given in Fig. 8 [2].

Wright remarks that when CRN models are constructed, whether by hand or by computer, and the results are then compared with carefully obtained diffraction data, as shown in Fig. 9 [32, 34] for a-Ge, none of the models fits all of the major experimental features to within experimental error, showing that there is probably still room for significant improvement in structural modeling. On the other hand, Zallen [2] also compares diffraction data with 


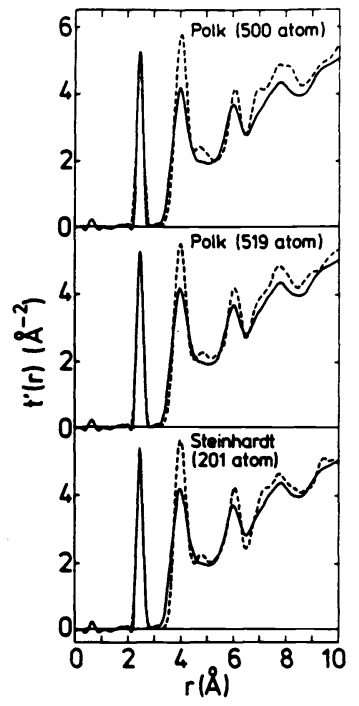

Fig. 9. Correlation functions for three different continuous random network models of amorphous Ge (dashed lines), compared with experimental results [32].

theoretical predictions for the refined versions of the tetrahedral CRN models, and for RCP models of metallic glasses. He finds that the agreement is "truly impressive" for the former, and "satisfactory overall" for the latter. This difference between the opinions of two prominent scientists who are experts in the field on just how satisfactory the agreement is points out the subjective nature of acceptability!

Synchotron radiation has recently become available, allowing researchers to perform $x$-ray diffraction experiments at any wavelength of their choice, rather than being dependent upon those wavelengths which are available from $x$-ray tube anode materials. This new flexibility greatly enhances the power of diffraction techniques [35]. For example, the Extended X-ray Absorption Fine Structure (EXAFS) method $[35,36]$, one of the several new structural tools which have come into its own after the availability of synchotron radiation, allows the acquisition of data on the average atomic arrangement around each particular type of atomic species in an alloy, rather than an average over the entire material. For example, an application of EXAFS to a-As ${ }_{2} \mathrm{Se}_{3}$ can yield information about the average local environments around the $A s$ and the Se atoms separately, rather than an average over both types of atoms. EXAFS experiments are likely to become increasingly prominent in the future, among the most powerful techniques for investigating the structures of non-crystalline alloys.

Recent work by Cervinka et al. [37] on the study of two-dimensional models of disordered structures by optical (Fraunhofer) diffraction, promises to contribute further to the understanding of $x$-ray and neutron scattering due to intermediate range ordering. Another new approach to understanding intermediate range order, based on IR absorption via vibrational modes, has been applied by Lucovsky and Wong [38] to amorphous selenium, and in particular, to the sequence of dihedral angles in this material. They find [38] that the chain model which best replicates the IR properties of a-Se is one in which the magnitude of the dihedral angle is fixed, while the phase of the dihedral angle ( \pm ) varies in a random way along the chain. In the absence of long range periodic order, understanding the extent and nature of intermediate range order is a challenging but crucial part of characterizing the structure and understanding the properties of a material. It will, therefore, be very fruitful to carry out detailed experimental and theoretical studies of intermediate range order for many different types of interesting non-crystalline materials. (See Sections III, VIII, and XI for further discussion and examples of intermediate range order.)

Data from measurements of optical and electrical properties can also provide valuable structural information [2,3]. Some useful types of experiments involve measurements of optical absorption, reflectivity or transmission as a function of photon energy; and measurements of photoluminescence, photoconductivity and electrical conductivity. These experiments provide information on electronic structure and on phonon (i.e., vibrational) energy distributions, which can then be used to draw conclusions about the underlying structure.

An example of how learning about electronic structure can help in understanding the underlying atomic-scale structure, is provided by considering the results of electron spin resonance (ESR) experiments. These experiments enable, for example, the observation of the number of unpaired spins on neutral dangling bonds in a-Si, via their characteristic ESR signal $(g=2.0055)$. One can then use this information to infer the concentration of dangling bonds present [16]. Such information on some of the prominent structural defects in the material is very important to any understanding of its physical properties. In considering an idealized structural model for non-crystalline solids, it has to be borne in mind that defects can play at least an equally important role in them as they do in crystalline solids. 


\section{ORDER AND DISORDER}

\section{'Amorphous' is not 'formless'}

Non-crystalline solids have traditionally been referred to as "amorphous" solids. This is unfortunate, because the word "amorphous", in its direct translation from its Greek roots (a-, without and morphe, form), means "without definite form" or "shapeless" [39]. It therefore, incorrectly implies that these materials lack any identifiable and well-defined types of order, making it hopelessly difficult to study them systematically.

In reality, nothing could be further from the truth. A brief review of the structural models presented in the previous section will immediately show that "amorphous" solids are far from "formless", but in fact, have well-defined types of order, albeit different from the monotonous repetitive patterns of crystalline solids. For example, the disappearance of periodic order in continuous random networks is associated with a spread in bond angles, while coordination numbers and bond lengths remain at their ideal values. The NMR studies on borate glasses [20] suggest the presence of even more order, since it is whole structural groupings, rather than individual atoms or small structural units, which are connected randomly. The molecular cluster network models [22] assume the presence of large morphologically and stoichiometrically distinct molecular clusters in glasses. The random close packing models [2] for amorphous metals also have order imposed by the constraints of the densest possible packing consistent with the impenetrability of the hard sphere atoms. For many transition metal/metalloid glasses (such as $\mathrm{Ni}_{3} \mathrm{~B}$ ), there seems to be even more ordering, with polyhedral building blocks such as tetrahedral, octahedral, or tricapped trigonal prismatic units of atoms forming the solid by random close packing [30]. Finally, in the random coils of a polymeric organic glass [31], the long and flexible coils are intermeshed in random-walk configurations as illustrated in Fig. 6, but each individual coil is formed by the repetition (up to $\sim 10^{5}$ times) of a monomeric molecular unit, so that the local environment of each atom, formed by those atoms (all within the same coil) to which it is bonded, is quite well-defined. An individual styrene molecule, and the bonding pattern in a coil of the polystyrene polymer [40] are illustrated in Fig. 10.

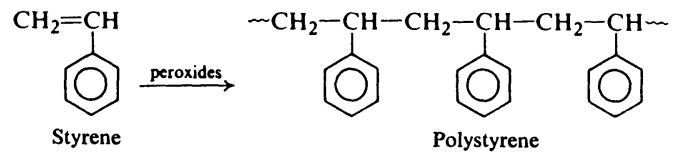

Fig. 10. (a) A molecule of styrene. (b) One coil of polystyrene, formed by many $\left(\sim 10^{5}\right)$ monomeric units of styrene, three of which are shown. Note the well-defined local environment of each atom in the coil.

\section{Importance of short range order}

As we have seen, the types of atomic-scale order observed in solids can be classified as short range (or local), intermediate (or medium) range, and long range, depending upon how far a predictable order extends when the arrangement of atoms is considered starting from a given atom or region in the solid.

Crystalline solids are characterized by long range periodic order. The atoms are located in a lattice generated by the repetition of a unit cell with a finite (usually small) number of atoms. The constraint of three-dimensional periodicity imposes the severe limitation of the existence of only seven crystal systems, 32 crystallographic point groups, and 230 space groups resulting from the application of the translational symmetry operations of the crystal [41].

This rigid long range periodic crystalline order, analogous to a regiment of soldiers in formation marching in a parade, is absent from non-crystalline solids by definition. A certain amount of intermediate range order may exist, however, as in the large and well-defined molecular clusters of the molecular cluster network models [22] for covalent glasses, the large structural groupings in borate glasses [20], the tricapped trigonal prismatic building blocks in transition metal/metalloid glasses [30] and the sequence of dihedral angles in a-Se [38]. Another interesting example of intermediate range order can be found in the hydrogenated and fluorinated amorphous silicon (a-Si:H:F) alloys [42] used for electronic applications (especially as solar cell materials). These alloys, when doped with moderate to large amounts of phosphorus to make them $n$-type semiconductors, manifest an intermediate range order similar to a microcrystalline state with a characteristic length (average particle size) of 20 to $60 \AA$, as observed from Raman, electrolyte-electroreflectance (EER) and transmission electron microscopy (TEM) experiments [43].

A very important factor in the determination of the structures and properties of non-crystalline solids is the short range order around the atoms in the material [21]. This local order can be considered in the context of a "total interactive environment" depending on several factors including the nearest-neighbor bonding, the effects of chemical forces, 
and the electrical charge distribution, all of which are reflected in the overall three-dimensional topology, in the nature of the electronic density of states distribution, and in various excitational processes [44]. This total interactive environment encompasses both the "normal structural bonding" consisting of the constituent atoms bonding with their optimal coordination numbers and bond types, and the deviations from these optimal coordination patterns, as represented by the "deviant electronic configurations" that are caused by the various types of defect states and are essential to understanding important phenomena such as the electrical transport properties $[45,46]$.

A useful systematic classification of the types of nearest neighbor environments about constituent atoms in a wide variety of non-crystalline inorganic solids has been presented by Messmer and Wong [33] who characterize these environments in terms of three parameters: (1) the bond angles sustained at a given atom by pairs of its nearest neighbors; (2) the bond lengths between a given atom and its nearest neighbors; and (3) the coordination number of a given atom. This classification scheme is very helpful in pointing out some of the major structural trends in non-crystalline inorganic solids, and in demonstrating the presence of systematic aspects in these "disordered" materials. For example, they observe that the well-defined short range order around the central atom ( $\mathrm{Si}, \mathrm{Ge}$ or $\mathrm{B}$ ) is preserved in simple oxide glasses such as $\mathrm{a}-\mathrm{SiO}_{2}, \mathrm{a}-\mathrm{GeO}_{2}$ and $\mathrm{a}-\mathrm{B}_{2} \mathrm{O}_{3}$, where very strong bonds are formed. The topological disorder in these glasses arises from variations in the $X-0-X$ angles over a wide range. In a majority of other non-crystalline solids, such as the elemental semiconductors (i.e., a-Si, a-Ge, a-As, a-Se, etc.) and compound semiconductors (such as a-GeSe ${ }_{2}, a-A_{2} S_{3}, a-A_{2} S_{3}$, etc.), the bond angles deviate from a single central value, while bond lengths and coordination numbers remain at their optimal values for most of the atoms in the material. In one glassy salt, a-BeF ${ }_{2}$, the three parameters follow the same trends as in the isostructural $\mathrm{a}-\mathrm{SiO}_{2} \cdot$ In another glassy salt $\left(\mathrm{a}-\mathrm{ZnCl}_{2}\right)$, only the bond angles around $\mathrm{Zn}$, but all three parameters around $\mathrm{Cl}$, vary over a range of values. Amorphous carbon (a-c) films formed by evaporation seem to be an intricate mixture of three-fold (graphitic) and four-fold (diamond-like) coordination. These films have an average $\mathrm{C}-\mathrm{C}$ bond length and $\mathrm{a} \mathrm{C}-\mathrm{C}-\mathrm{C}$ bond angle intermediate between those of diamond and graphite, and a fractional average first coordination number between 3 and 4 . Metallic glasses such as Fe-P and Pd-Si alloys are characterized by ranges of values for all three parameters around the metallic atoms ( $F e, P d)$, and well-defined sets of values for the bond lengths and coordination numbers but ranges of values for the bond angles around the metalloid atoms ( $P, S i)$. In general, more short range order parameters appear to be preserved around those atoms and in those systems where the bonding is more strongly directional [33].

\section{Network momentum}

We have already mentioned that the lack of a periodic structure makes it impossible to use the standard concepts and techniques of crystalline band theory (such as Brillouin zones, Bloch functions, $k$-space, etc.) which have been so useful in the understanding of crystalline solids [47]. It is worth considering, however, whether the presence of at least a quantifiable short range order in non-crystalline solids can be utilized to define an analog of crystal momentum. It seems that such a generalization can be carried out, leading to the idea of "network momentum" [48], an observable which commutes with the Hamiltonian for the system. This quantity arises from order in the local environments of the atoms, without requiring any long range order or overall structural symmetry. The network momentum can be used as a conserved quantum number to classify stationary states of definite network momentum. The Bloch waves of a crystal can be replaced by "network waves". The generalization of the Brillouin zone, i.e., the set of possible values of network momentum, is the "reciprocal network".

These generalizations have been applied $[48,49]$ to systems with tight binding Hamiltonians of the Thorpe-Weaire model [50] for networks of constant valency, and for extensions of this model, useful for studying the electronic states of disordered solids. For example, a three-fold coordinated model system, whose electronic basis set is taken to be the $\mathrm{sp}^{2}$ hybrid orbitals directed along each bond, has been examined [48]. Future research will show whether it will be possible to define infinite networks related to real materials, carry out this type of analysis, and obtain physical insights as useful in understanding non-crystalline solids, as the standard momentum space description has been in understanding crystalline solids. At present, this seems like a possibility worth exploring further. It seems, nevertheless, likely that the information that can be gained from the application of such considerations to non-crystalline solids will never equal the amount, unambiguity and accuracy of the information available from the momentum space description of crystalline solids, whose structures can be better defined.

\section{Icosahedral order}

The question of the possible existence and properties of materials with icosahedral patterns of atomic arrangement has recently become one of the most exciting fields of research in solid state physics. The new understanding that is emerging out of this work not only presents deeper insights into the nature of order and disorder but also seems to hold the 
promise of providing powerful new theoretical techniques that can be utilized to understand a wide variety of structures within a unified formalism, and to discover hidden order in non-crystalline structures.

An example of a perfect icosahedron occurring in chemistry can be found in the structures of the boron hydrides [51,52]. Many fascinating polyhedral arrangements are possible with the general formula $\mathrm{B}_{n} \mathrm{H}_{n}^{\mathrm{C}}$, where the boron atoms occupy the vertices of a polyhedron, the hydrogen atoms are directed outwards, and $c=-2$ in the vast majority of cases. By far, the most stable of these polyhedra occurs for the icosahedral structure with $n=12$ [52], as shown in Fig. 11 .

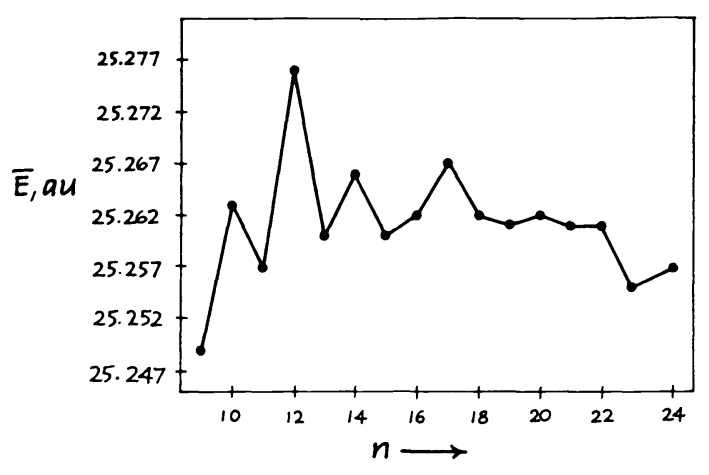

Fig. 11. Calculated energies per $B H$ unit $(\bar{E}=|E| / n)$ as a function of $n$ for the preferred polyhedral $\mathrm{B}_{n} \mathrm{H}_{\mathrm{n}}^{2-}$ structures [52].

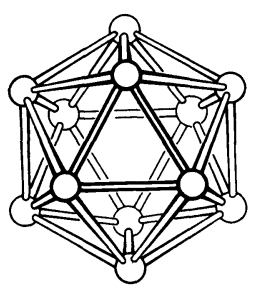

(a)

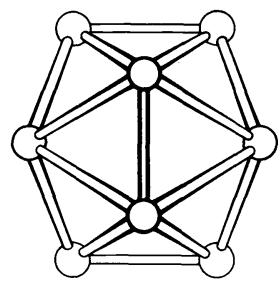

(b)

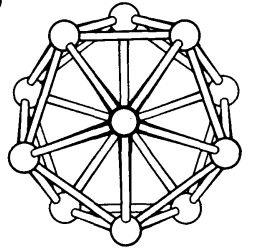

(c)

Fig. 12. Three views of a regular icosahedron [53]: (a) Looking down a face. There are ten three-fold axes of symmetry connecting the midpoints of opposite triangular faces. (b) Looking down an edge. There are 15 two-fold axes connecting the midpoints of opposite edges. (c) Looking down a vertex. There are six five-fold axes connecting vertices at opposite ends.

Although the perfect icosahedron is a highly symmetric and very dense arrangement of atoms, full icosahedral order is not possible in a crystal. The reason for this becomes clear when we look at the three views of a regular icosahedron, as presented in Fig. 12 [53]. As the view looking down from a vertex shows, an icosahedron possesses six five-fold symmetry axes, each one connecting two vertices at opposite ends. It can easily be shown [41] that only one-, two-, three-, four-, or six-fold axes of rotation are consistent with full three-dimensional translational periodicity and therefore possible in a crystalline solid. Full icosahedral order is therefore impossible. Any crystalline structure, where the local bonding requirements can be best satisfied by icosahedral arrangements of atoms, has a point group and a space group which are lowered from perfect icosahedral symmetry by distortions of the icosahedra and/or connections of lower symmetry between the icosahedra. This can be seen from the structure of elemental crystalline boron, where the most stable tetragonal and rhombohedral allotropic forms contain units of nearly (but not exactly) regular icosahedra, with various types of connections between these distorted icosahedra [53]. Electron diffraction experiments on vacuum-deposited thin films of amorphous boron produced by evaporation show that icosahedra are also the predominant structural units in a-B [54]. The major difference from the crystalline phases is that these icosahedral units form a three-dimensional continuous random network through quite random arrangements of the icosahedra, with a random (disordered) distribution of the possible types of connections between the icosahedral building blocks [54]. (This is also another example of an amorphous material with intermediate range order, on the scale of the well-defined icosahedral units!)

The Frank-Kasper phases [55] of transition metal alloys are another example of broken icosahedral symmetry. In these intermetallic crystals, the smaller transition metal element at the center becomes surrounded by twelve larger atoms arranged like the corners of an icosahedron. Distortions and different orientations of several icosahedra in a unit cell then reduce the symmetry of the crystal to a point group and a space group consistent with the existence of three-dimensional translational periodicity. 
Finally, it was pointed out a long time ago [56] that an icosahedral clustering of twelve atoms about a central sphere is energetically preferable to crystalline packings for a standard Lennard-Jones pair potential. This suggested that the short range order in simple undercooled liquids and metallic glasses is predominantly icosahedral.

\section{Orientational order without translational periodicity}

It is possible for an arrangement of atoms to lack translational periodicity, but still have orientational order, with long range correlations in the orientations of "bonds" connecting nearest-neighboring atoms (bond-orientational order) or of molecular units (molecule-orientational order).

Liquid crystals [57-60] are a well-known class of systems where very complicated types of orientational order are important. These materials consist of long, rod-like organic molecules which align themselves in complex patterns. They have proved useful as displays in devices such as calculators and watches. They also hold promise for use in conjunction with thin-film semiconductors in large-area flat panel displays. In addition, liquid crystal phases are commonly encountered in biologically active systems, and are therefore important in biophysics.

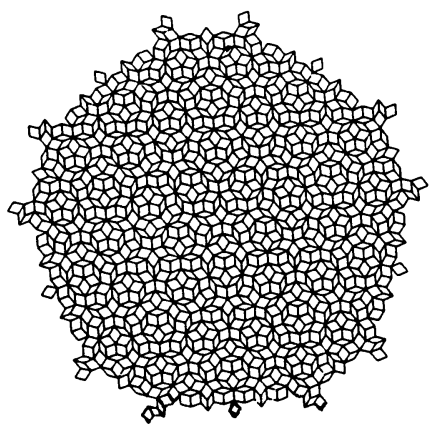

Fig. 13. Portion of a two-dimensional Penrose tiling $[61,62]$, illustrating a non-periodic system with perfect bond-orientational order.

Systems with icosahedral order, which can not have translational periodicity, can nevertheless have bond-orientational ordering. A simple example of how this might happen is presented in Fig. 13 [61] for the two-dimensional Penrose tiling [62]. There are two different "unit cells" in these tilings, namely the two different kinds of rhombuses, whose packing generates the pattern depicted in Fig. 13. These tilings have manifest pentagonal symmetry. Although the tile edges all lie along the axes of a regular decagon and the tiling therefore has perfect decagonal bond-orientational order, it is not periodic. A similar tiling in three dimensions by two polyhedral tiles that fill space in a way that yields long range bond-orientational order without translational periodicity could form a model for quasi-periodic structures with icosahedral order [61].

\section{Icosahedral quasi-crystals}

The possibility of an icosahedral phase with long range orientational order was first inferred from computer simulations using the Molecular Dynamics technique [63,64]. A first-order liquid-to-icosahedral phase transition was predicted from mean field theory calculations $[64,65]$. These papers were excellent examples of major theoretical predictions preceding experimental observation and leading the way in breaking new ground in materials research, rather than following and trying to explain the experimental observations.

A very significant breakthrough occurred with the electron diffraction experiments of Shechtman et al. [66], which indicated the existence of a metallic solid which diffracts electrons with a very sharp pattern of diffraction spots like a single crystal, but has an icosahedral point group symmetry inconsistent with the lattice translations of three-dimensional periodicity. Grains of up to $2 \mu \mathrm{m}$ in size with this structure form in rapidly cooled alloys of $\mathrm{Al}$ with $10-14 \% \mathrm{Mn}$, $\mathrm{Fe}$, or $\mathrm{Cr}$ [66] and entire grains appear to have long range orientational order. The icosahedral phase forms during rapid cooling of the melt by a nucleation and growth mechanism characteristic of a first-order phase transition, and it is a truly metastable phase which is remarkably resistant to crystallization.

These quasi-periodic systems have been named "quasi-crystals" by Levine and Steinhardt $[61,67]$. They are currently the subject of intense theoretical research aimed at understanding their structures, properties and phase transitions, and their place in the general context of classes of materials [61,67-71]. For example, it was shown [69], using a theory of first-order phase transitions based on Jahn-Teller distortions [72], and the results of SCF-X $\alpha-S W$ molecular orbital calculations on clusters representative of the local crystalline environments of aluminum and Al-Mn alloys, that Jahn-Teller-like distortions can account for both melting and glass formation. The simple chemical arguments on which this theory is based can also be used to explain the observed stability of icosahedral structures in Al-Mn alloys [69]. These calculations are especially worthy of study by theoretical 
chemists, since they illustrate how the standard computational techniques of molecular orbital theory can be utilized to gain insights into novel structures.

The ideal composition and structure of the icosahedral quasi-crystal phase have been inferred from the known phase diagrams of aluminum-transition metal alloys, and tilings in three dimensions by rhombohedral tiles to produce the phases of interest have been discussed [70].

The mean field theory and density functional theory treatments of quasi-crystals have been considered in the context of the ordering in condensed phases produced by orientational freezing in three dimensions [71]. The mean field theory treatment enables connections to be made with theories of biaxial ordering in liquid crystals. The density functional theory treatment has the advantage of not having any adjustable or unknown parameters. The stability of the orientationally ordered phase, its density, and the entropy change upon its formation, are all seen [71] to be determined, to first order in thermodynamic perturbation theory, by the structure factor of the corresponding liquid phase. These approaches represent the state-of-the-art and are also very valuable in learning more about icosahedral order; however, they do not constitute easy and obvious entry points into the field for theoretical chemists more accustomed to the traditional techniques of computational chemistry.

It has recently been shown [73] that the electron diffraction data [66] can also be

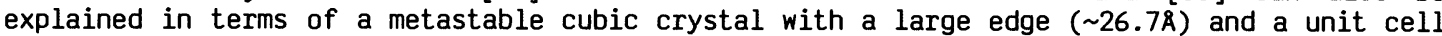
containing approximately 1,120 atoms. This alternative explanation, which is firmly grounded in traditional crystallography, does not require the postulation of the existence of "quasi-crystals" as a new kind of matter. More accurate x-ray diffraction experiments might help in deciding between these two explanations, one of which has a beautiful conceptual framework, and the other one has the accumulated wisdom of traditional crystallography, in its favor.

\section{The curved space approach}

A unified formal approach to understanding the structures of many classes of materials with different types and amounts of order can be provided by viewing them as arising from projections of regular polyhedra (polytopes) in a curved space onto the usual flat three-dimensional Euclidean space $[68,74]$.

An example of a very simple projection from a two-dimensional space (a square lattice) to a one-dimensional sub-space (a line of incommensurate slope which passes through the origin but through no other lattice point), subject to a well-defined projection rule, is illustrated in Fig. 14 [4]. All lattice points within a fixed distance of the line are projected on it, defining the atom-center positions on it. Although the number of possible ways one can project between spaces is much larger in higher dimensional spaces (whether flat or curved), and the projection rules are far more complicated, Fig. 14 illustrates the general idea.

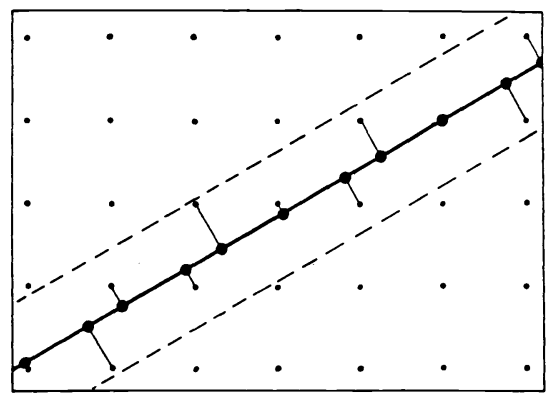

Fig. 14. Projection from a two-dimensional square lattice onto a one-dimensional subspace (a line). The solid dots are projected images of those square lattice sites which lie between the dashed lines. Since the line has an incommensurate slope and passes through the origin but through no other lattice point of the square lattice, the site distribution obtained on the line is only quasi-periodic, and corresponds to a one-dimensional quasi-crystal [4].

The curved space approach [74] examines hidden order in non-crystalline structures in the light of topological and geometrical limitations, arising from the requirements of space filling, imposed on the optimal short range order which would completely determine the structure if the local interactions between atoms were the only important factors.

The failure of certain simple structural patterns, which would normally be preferred and provide a dense and highly favored packing, to regularly fill (or "tile") the space, is referred to as "frustration" [68,74]. A very important example of frustration (see Fig. 15) is given by the fact that regular tetrahedra do not tile three-dimensional Euclidean space. The cause of this frustration is that the dihedral angle of a tetrahedron $\left(70.5^{0}\right.$ ) is not a sub-multiple of $2 \pi$ (as $72^{\circ}$ is), causing a misfit when one tries to propagate the tetrahedral local configuration by completely surrounding a given bond with five tetrahedra, or form an icosahedron by packing tetrahedra. 

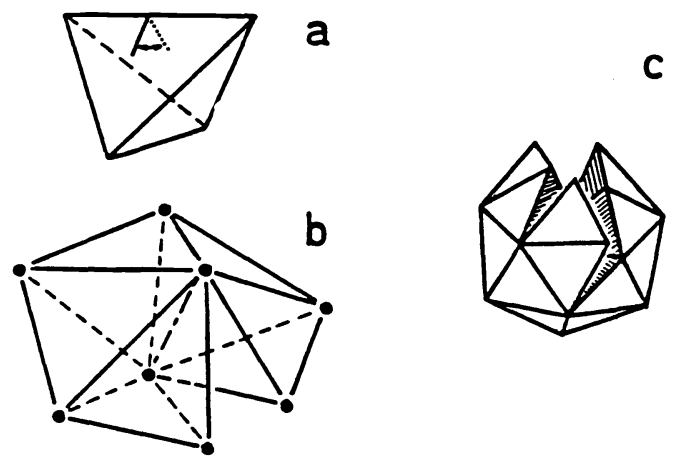

Fig. 15. Regular tetrahedra do not tile three-dimensional Euclidean space, resulting in "frustration" [74]: (a) A regular tetrahedron. (b) The failure of five regular tetrahedra to completely surround a given bond. (c) The resulting failure of the packing of tetrahedra to form a perfect icosahedron. One gets pseudo-icosahedral (dense packing or polytetrahedral) models out of such a packing.

On the other hand, what is known as "polytope $120 "[4,74]$ or "polytope $\{3,3,5\} "$, is a regular polyhedron ("Platonic solid") in four dimensions. Its two-dimensional projection is shown in Fig. 16. It has 120 vertices, all lying on the surface of a four-dimensional sphere, which defines a curved non-Euclidean three-dimensional space, just as the surface of

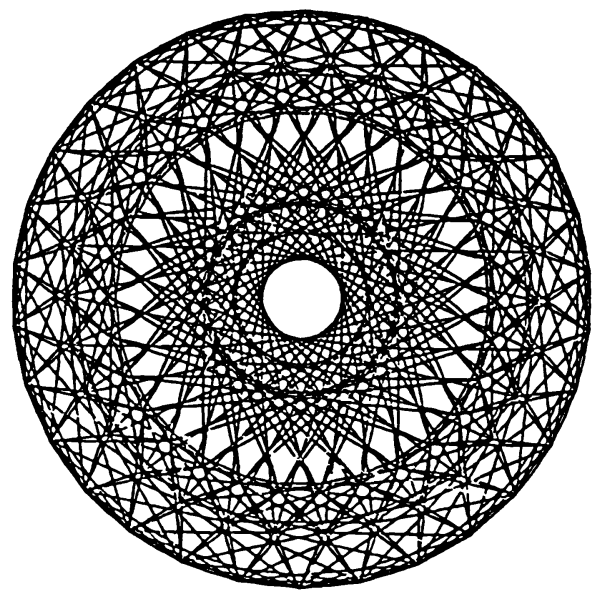

Fig. 16. Two-dimensional projection of polytope $\{3,3,5\}[68]$.

a regular sphere defines a curved non-Euclidean two-dimensional space. Within this curved three-dimensional space, the 120-vertex polytope forms a configuration in which five tetrahedra fit perfectly around each bond, so that 600 tetrahedra completely tile this curved space. Local dense packing is thus maintained everywhere. Topological frustration is removed, at the expense of the introduction of curvature [4].

The mapping which is then carried out on the structure in curved space to generate a projection to Euclidean space, is called "flattening", and can be likened to peeling an orange by spreading out its crust on a plate. The localized distortions (i.e., defects) caused by the mapping from a curved to a flat space, are called "disclinations" [74]. A complete set of disclination lines, which forms a network, can cancel the curvature and completely define the structure in Euclidean space. Many seemingly unrelated types of structures can be generated by applying different types of disclination networks to the $\{3,3,5\}$ polytope or to other polytopes. This is performed by cutting the structure of the polytope and adding or removing a wedge of material between the two lips of the cut [74].

The following types of structures are among those that have been treated thus far within this unified framework:

1. The Frank-Kasper phases [55], including the Laves phase alloys such as $\mathrm{MgZn}_{2}$, $\mathrm{MgCu}_{2}$ and $\mathrm{MgNi}_{2}$, are among the types of crystalline solids which can be generated $[68,74]$. 
2. The cubic $A 15$ (or $\beta$-tungsten) structure, which is a type of Frank-Kasper phase represented by alloys such as $\mathrm{W}_{3} \mathrm{O}, \mathrm{W}_{3} \mathrm{Si}, \mathrm{AlV}_{3}, \mathrm{AuTi}_{3}, \mathrm{CrO}_{3}$, etc. [75] can also be generated $[68,74]$. These $A 15$ structures are especially important because the superconductors with the highest critical temperatures (such as $\mathrm{Nb}_{3} \mathrm{Ge}$ and $\mathrm{Nb}_{3} \mathrm{Sn}$ ) are metastable Al5 phases [76].

3. Amorphous metallic alloys can be generated by using non-periodic networks of disclination lines [74].

4. Covalently bonded amorphous semiconductor alloys (such as a-Si:H) can also be modeled [77], although the existence of inhomogeneities in such systems presents a problem.

5. Icosahedral quasi-crystals can be described in terms of the iterative flattening of polytopes via hierarchical disclination networks [78].

6. Finally, the defects that introduce octahedral local configurations into RCP models of amorphous metals, which can be predominantly regarded as random packings of tetrahedra, are related to disclinations, and are referred to as "screw" axes or "dispiration" [79].

The curved space approach is a promising technique for the analysis of amorphous structures of simple stoichiometric compounds, although it would not be expected to give much insight into complex multicomponent alloys. It also remains to be decided whether the approach has real physical content, or whether it should be viewed more as a mathematical technique unifying the treatment of these diverse structures in a mainly formalistic sense.

\section{MODEL SYSTEMS OF LOW DIMENSIONALITY}

Real materials, of course, have a fully three-dimensional structure, although quasi-two-dimensional arrays, e.g., graphite, and quasi-one-dimensional arrays, e.g., TTF-TCNQ, do exist. In any event, simple model systems with reduced dimensionality can be studied with more accuracy than three-dimensional networks, and thus can provide useful information about real materials provided that the right questions are asked [59]. For example, under rather general conditions, concerning the range of the interparticle forces, it can be shown that spontaneous crystalline order should not exist in one or two dimensions. This very important theorem serves as a reminder that the real materials, such as graphite and TTF-TCNQ, are indeed three - dimensional, even though it might be both a convenient and a reasonable approximate model, at least for solving certain types of physical problems, to view them as consisting of two-dimensional or one-dimensional arrays respectively, embedded in the three-dimensional space. The application of similar considerations to spin correlation functions shows that, in the absence of a finite magnetic field or magnetic anisotropy, spontaneous ferromagnetic or anti-ferromagnetic order should be thermally unstable, and therefore should not appear, in one or two dimensions [59]. Several model systems that have shed some light on specific unsolved problems have been investigated recently, and are discussed in the remainder of this section.

One of the major unanswered questions is whether the non-crystalline atomic arrangement in a glass is indeed a metastable equilibrium configuration that minimizes the interaction potential. This has been shown to be the case for models with a small number of particles [28], but has not been demonstrated for large systems. Schilling and Reichert [80] recently considered an anharmonic one-dimensional chain with next-nearest-neighbor interactions that introduce structural frustration. By introducing spatial chaos into the interactions via a random variable in the potential energy, they were able to demonstrate that: (1) no long-range order exists; (2) the structure factor contains extra peaks at special values of the wave number, $k$, characteristic of short-range order; and (3) the density of states is time-varying, leading to a power-law behavior of the specific heat that has been observed in some real glasses [81].

A second unsolved problem is that of the dc conductivity of a material with static disorder [82]. For one-dimensional solids, the exponential localization resulting from elastic scattering leads to conductivity fluctuations even in nominally identical samples. Mello and Kumar [83] applied the techniques of information theory to this problem, maximizing the entropy subject to flux conservation, time-reversal invariance, and a particular scaling law, and were able to calculate the movements of the resistivity in the limit in wich the length of the wire is long compared to the localization length. More work is necessary to extend their solution to smaller wire lengths. 
Another problem concerns the calculations of the configurational entropy of a CRN, a difficult analysis to carry out in general. Some recent progress has been made by Klenin and Promislow [84], who considered a two-dimensional case in which the four-particle correlation function was constrained to take on only discrete values. The structure analyzed was a two-fold and four-fold coordinated array of rectangular blocks with a $60^{\circ}$ bond angle at the four-fold-coordinated sites. They found sharp maxima in the entropy per block at two-fold-coordinated sites with bond angles of $120^{\circ}$ and $155^{\circ}$. For the former case, the blocks form a hexagonal grid, while for the latter, a specific ring structure appears. In both cases, the entropy maxima signify a relative lack of interference within the conditions of dense packing. Such models clearly give some insight into the general nature of random networks. It is interesting to speculate that the possibility of doping amorphous semiconductors arises from the tendency of CRN's to choose the bonding configurations that avoid interference between atoms.

\section{CHEMICAL BONDING CONSIDERATIONS}

\section{The importance of chemical bonding considerations}

We have already made use of chemical bonding considerations in our discussions of the structural models used in describing non-crystalline solids (Section II) and of the nature and extent of order in disordered structures (Section III). The short range order in an amorphous solid is primarily determined by the local chemical bonding requirements of its constituent atoms. The extension of these local configurations into three-dimensional solids generates structures which can be described by a variety of models such as CRN, molecular cluster network and RCP models. The intermediate range order observed in some materials, as manifested in the large structural groupings in $\mathrm{a}-\mathrm{B}_{2} \mathrm{O}_{3}[20]$, the tricapped trigonal prismatic building blocks in transition metal/metalloid glasses such as $\mathrm{Ni}_{3} \mathrm{~B}$ [30], the sequence of dihedral angles in a-Se [38], the microcrystalline-like order in a-Si:H:F alloys doped with moderate to large amounts of phosphorus [43], and the icosahedral structural units in a-B [54], is also primarily due to the special stability of such structural configurations where the chemical bonding requirements of the constituent atoms are well-satisfied. On the other hand, it was seen to be impossible to completely fill space by packing some simple structural units, such as tetrahedra, which are often the favored types of short range order for a given set of constituent atoms. This "topological frustration", which often prevents the optimum local environment from being fully propagated in three dimensions, was seen to possibly form the basis for a whole new and unified framework for understanding many types of materials.

The purpose of the present section is to focus in more detail on the chemical bonding considerations of importance in understanding non-crystalline solids. There is clearly a lot that chemists can contribute to the understanding of non-crystalline solids, along the lines of the work described in this section.

It is possible to synthesize amorphous materials that have no crystalline analogues, but possess interesting and even unique physical properties [21]. This extra degree of compositional freedom, which introduces a whole new dimension to the science and technology of non-crystalline solids, is due to the types of chemical bond distributions possible in a structure in which long range order is absent. These considerations have been presented [85] in the form of seven "basic rules of amorphicity", which summarize the basic "anti-crystalline" chemical bonding configurations and their structural and physical implications. The most important conclusion of this work [85] is that deviations from local order, as reflected by the deviant electronic configurations and the total interactive environments, control the fundamental physical properties (such as transport properties) of non-crystalline materials just as perturbations of periodicity control those of crystalline materials.

\section{Glassy alloys}

Threshold switching alloys such as $\mathrm{Si}_{18} \mathrm{Ge}_{7} \mathrm{As}_{35} \mathrm{Te}_{40}$, and memory switching alloys such as $\mathrm{Ge}_{15} \mathrm{Te}_{81} \mathrm{Sb}_{2} \mathrm{~S}_{2}$ and $\mathrm{Ge}_{24} \mathrm{Te}_{72} \mathrm{Sb}_{2} \mathrm{~S}_{2}$, have a special place among amorphous solids with nocrystalline analogues. The discovery of reversible switching phenomena in such alloys [86], in which the motivating potential can either be electrical (application of a large voltage, resulting in an increase of electrical conductivity by several orders of magnitude), or optical (application of laser or xenon flash lamp pulses, resulting in a sharp change in reflectivity), induced a rapid increase in both basic and applied research on amorphous solids.

The most important short range order parameters in the description of structure and bonding in glassy alloys, such as in the three compositions mentioned above, are the coordination numbers $C$ of the elements entering the alloy and the bond energies $D$ between the elements. 


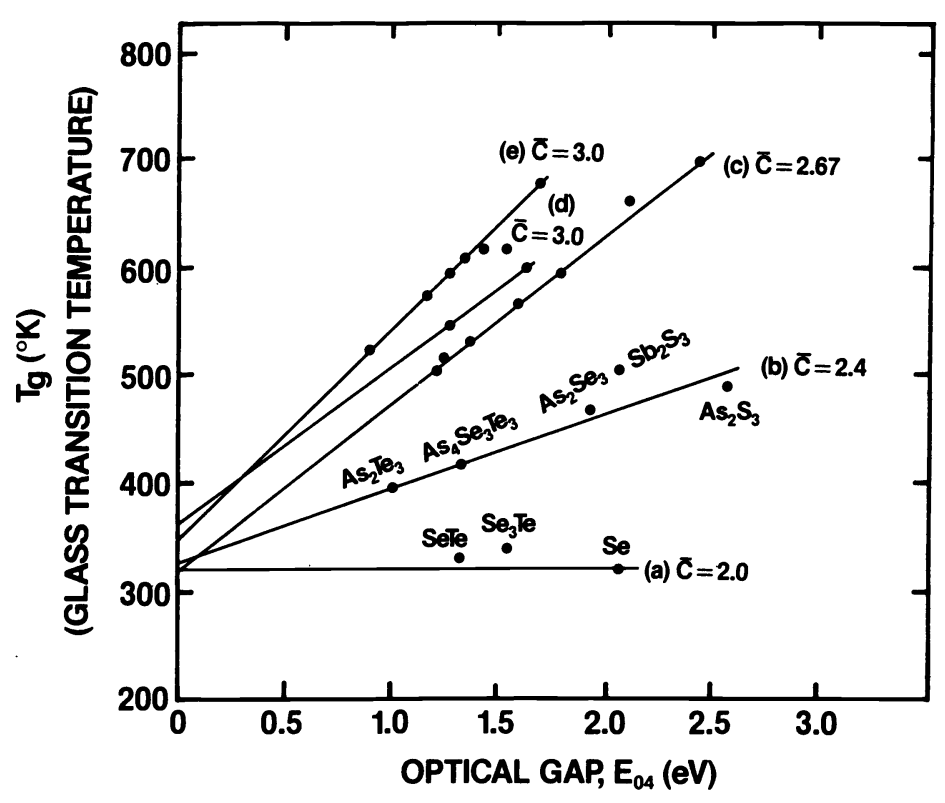

Fig. 17. The glass transition temperature $T_{g}$ as a function of the optical gap energy $E_{04}$ for alloy compositions with fixed values of the average coordination number $\bar{C}[87]$.

An elegant experimental demonstration of the importance of these parameters has been provided by deNeufville and Rockstad [87] in their empirical "T $\mathrm{g}_{\mathrm{g}}-\overline{\mathrm{C}}$ Correlation":

$$
\mathrm{T}_{\mathbf{g}} \approx \mathrm{T}_{\mathrm{g}}^{\mathrm{0}}+\beta(\overline{\mathrm{C}}-2) \mathrm{E}_{04}
$$

illustrated in Fig. 17. Here, $T_{g}$ is the glass transition temperature of the alloy; $\mathrm{T}_{\mathrm{g}}^{0} \approx 340 \pm 20^{\circ} ; \overline{\mathrm{C}}$ is the average coordination number; $\mathrm{E}_{04}$ is the energy at which the optical absorption coefficient is $10^{4} \mathrm{~cm}^{-1}$ and is a measure of the optical gap $E_{g}$ and of the

energies of the weakest bonds in the alloy; and $\beta$ is a linear proportionality constant for each value of $\bar{C}$. Clearly, $T_{g}$ increases (i.e., the glass becomes more stable) both as $\bar{C}$ (cross-linking) increases, and as $\mathrm{E}_{04}$ (an index of the strengths of the weakest bonds) increases. The "T $-E_{g}-\bar{C}$ Correlation" is discussed further in Section VIII, as an example of the importance of network rigidity in alloy glasses.

A semi-quantitative approach which uses these coordination number and bond energy parameters and some simple rules for obtaining optimal chemical bond distributions in a glassy alloy, has been discussed by Bicerano and Ovshinsky [88]. The only assumptions used in this simple model involve: (1) the preferred coordination numbers of the elements; (2) the maximum amount of chemical ordering possible, to determine gross overall structural features; and (3) the formation of bonds in the sequence of decreasing bond energy. This simple approach provides useful information concerning $T_{g}$, cohesive energies, the numbers and compositions of phases expected to be present, bond distributions, and electrical properties such as the conductivity and switching phenomena.

Phillips [89] has presented calculations of the experimental heats of formation of crystalline $\mathrm{As}_{2} \mathrm{X}_{3}(\mathrm{X}=\mathrm{S}, \mathrm{Se}, \mathrm{Te})$ and $\mathrm{GeSe}_{2}$ based on chemical bonding considerations related to the competition between charge transfer and lone pair resonance screening. Similar considerations can also be useful for the amorphous analogues of these and other similar types of alloys, especially since "metastable" amorphous local minima on a potential surface are usually only slightly higher in Gibbs free energy than the corresponding crystalline minima for the same material.

\section{Materials with predominantly tetrahedral coordination}

Elemental tetrahedrally bonded non-crystalline solids such as a-Si and a-Ge have extremely high concentrations of point defects (e.g., dangling bonds), making them unsuitable for semiconductor device applications, including thin film transistors and solar cells. To 
overcome this problem, these elements are alloyed, usually with $H$ [90], or with both $H$ and $F$ [42]. These alloying elements, which have low coordination numbers, decrease the strain energy of the tetrahedral network by reducing its average coordination number, and also act as dangling bond terminators [21,85]. As a result, semiconducting materials of much lower intrinsic defect density and higher quality are obtained.

Grigorovici and Gartner [91] have used non-equivalent s-p-d hybrid bonds to quantitatively describe the local angular and radial distortions of elemental metastable crystals and random network models of the non-crystalline phase in tetrahedrally bonded elemental solids such as $\mathrm{Si}$ and Ge. This approach is simjlar to the point of view used in crystal chemistry, where the bonds are supposed to adapt themselves to the new geometry in order to eliminate strain. It is shown [91] to be preferable to the alternative model of an elastically strained structure with invariable chemical bonding. The sizes of the small d orbital contributions seem to primarily determine the amount of energy stored in a-Si and a-Ge, so that in this model, elastic strain is replaced by electronic excitation, namely, by the promotion of valence electrons to $d$ orbitals in different quantities.

Manaila et al. [92] have used EXAFS phase shift experiments to examine the differences in chemical bonding between crystalline and amorphous Ge. They observe that the total phase shifts for Ge-Ge pairs in c-Ge and in a-Ge are different, so that these phase shifts are sensitive to whether the structure is crystalline or amorphous. Their work is another example of the growing use of the EXAFS technique $[35,36]$ for studying structure and bonding in the non-crystalline state.

Popescu [93] has attempted to explain the major deviations from linearity observed in physical properties when two monovalent alloying elements such as $\mathrm{H}$ and $\mathrm{F}$, or $\mathrm{H}$ and $\mathrm{Cl}$, are simultaneously included in thin films of $\mathrm{a}-\mathrm{Si}$, as compared with the cases when they are included separately. He uses a random network model in which the bound alloying elements involve different sites of the network. In this model, only $\mathrm{H}$ can penetrate in small voids, while $\mathrm{F}$ or $\mathrm{Cl}$ usually satisfy surface dangling bonds or eliminate strained bonds in bigger voids. The conclusion drawn from this work is that chemical bonding considerations (in this case, the sizes of the halogen atoms and the strengths of the silicon-halogen bonds), are the key factors governing the behavior of these materials.

\section{Metallic systems}

An interesting example of the use of chemical bonding considerations in non-periodic metallic systems has recently been provided by McAdon and Goddard [94], who examined numerous $\mathrm{Li}$ atom clusters (up to 13 atoms) with the generalized valence bond (GVB) method $[95,96]$. They concluded [94] that the optimum metallic bonding involves singly occupied orbitals localized interstitially, i.e., in bond midpoints, triangular hollows, and tetrahedra. For $\mathrm{Li}_{13}^{+}$, the low energy isomers have local five-fold symmetry axes (as in an icosahedron), but lead to low overall symmetry. The guiding principle is that the structures optimize the number of tetrahedral hollows while keeping the sharing of vertices below a given threshold. The optimum structures are significantly more stable than the high-symmetry icosahedral, hcp-like and fcc-like clusters. McAdon and Goddard [94] suggest that these results will form a useful starting point in developing valence bond ideas for predicting geometries, electronic structures, and properties of metallic clusters, and for describing localized phenomena in solids, such as the nature of defects or interfaces, as well as providing useful conceptual ideas for predicting the chemistry and catalytic properties of such systems and for predicting the factors controlling stabilization of amorphous systems.

Recent research on the universal features of bonding in metals, including the demonstration of a simple two-parameter scaling under which binding energy-distance relations for metallic systems exhibit a universal behavior, suggest a commonality of metallic bonding as well as a close relationship between molecular and metallic bonding [97]. Similar scaling considerations might also be useful for understanding amorphous metallic systems.

Another very significant recent contribution to understanding chemical bonding in metallic systems is the work of Gelatt et al. [98] on the theory of the chemical bond in crystalline transition metal/metalloid alloys. These authors show that rather different types of bonding result when the atomic $s$ and $p$ levels of the non-transition element lie above, below, or near the transition metal d level. They also show that the heats of formation of these alloys result from the competition between two simple physical effects: (1) the weakening of the transition metal bonds by the lattice dilation required for the accommodation of the larger non-transition element, and (2) the increased bonding which results from the occupation of the bonding members of the hybrid states formed from the interaction between the transition metal $d$ states and the $s$ and $p$ states on the non-transition element. Although this work was carried out within a crystalline framework, the resulting chemical insights are clearly also useful for understanding analogous non-crystalline alloys. 


\section{THERMODYNAMIC AND KINETIC CONSIDERATIONS}

It is a basic tenet of modern science that any collection of atoms arrange themselves so as to attain the state of lowest Gibbs Free Energy, G, at the ambient temperature and pressure; however, this turns out to be somewhat naive when applied to the solid state. In fact, it is not clear that any non-crystalline solid attains the state of absolutely lowest $G$ at any temperature and pressure. By definition, a solid is a material whose shear viscosity exceeds the order of $10^{4} \mathrm{~N}-\mathrm{s} / \mathrm{m}^{2}$. Such a viscosity implies that a shear stress of $1 \mathrm{~N}$ applied to a $1 \mathrm{~cm}$ cube of material would take about a year to produce a displacement of 30 $\mu \mathrm{m}$, a barely measurable deformation. Although this definition is completely arbitrary, few would deny that a system with an atomic relaxation time of the order of a year is indeed solid. In contrast, typical liquids have viscosities less than $1 \mathrm{~N}-\mathrm{s} / \mathrm{m}^{2}$, so there is ordinarily no difficulty in differentiating between the two phases; however, it is precisely the lack of atomic mobility in solids that enables the formation of metastable phases, for which $G$ is a local but not a global minimum. Upon rapid cooling, thermal energy is extracted from the system and metastable structures are "frozen in". A typical situation is sketched in Fig. 18 [2]. As the temperature of a liquid is reduced, one of two things can happen, depending on the cooling rate: it can solidify discontinuously via a first-order phase transition to a crystal (curve a in the sketch), or continuously via a higher-order phase transition to a glass (curve b). In the latter case, appropriate to rapid cooling, there is a region in which the liquid is supercooled, but at the glass-transition temperature, $T_{q}$, the viscosity increases by many orders of magnitude to form a solid, identifiable by the characteristically low thermal-expansion coefficient (as well as by the large relaxation times, often exceeding the age of the universe). Even crystals can be metastable: a diamond may be forever, but if the universe lasts sufficiently long, it will eventually convert to graphite. In popular usage, a non-crystalline solid capable of being quenched from the liquid phase is called a glass. All glasses have a $T_{g}$, although its value may vary somewhat with the cooling rate. Some non-crystalline solids (e.g., Si) can be formed only by direct condensation from the gas phase; they are usually referred to as amorphous solids. Such systems are not ordinarily describable in terms of thermodynamic equilibria; instead, growth mechanisms and relaxation effects must be handled on a kinetic basis.

The details of the glass transition have remained elusive right up through the present, and remain a fertile subject of research. Gibbs and DiMarzio [99] provided a start by considering a one-dimensional polymer and identifying $\mathrm{T}_{\mathrm{g}}$ with the temperature at which the configurational entropy vanishes. An alternative approach is the free-volume theory of

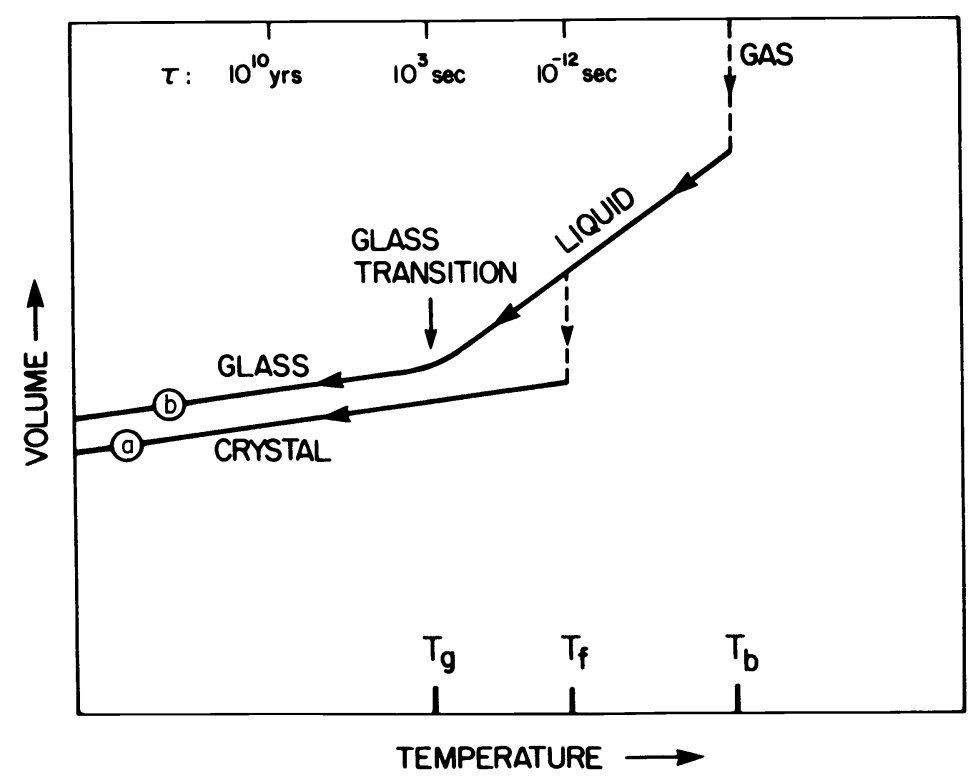

Fig. 18. The two cooling paths yielding solids:

(a) slow cooling, producing a crystal;

(b) rapid cooling, resulting in a glass [2]. 
Cohen and Turnbull [100], which focuses on the volume available for the atoms (treated as hard spheres) to move within their environment in the liquid phase. The glass transition is then identified as the point at which the unoccupied space (the free volume) drops below a critical fraction of the total volume. A further discussion of the glass transition appears in Section XII.

Clearly, thermodynamic and kinetic considerations are central to an understanding of the formation and structure of non-crystalline solids. One of the prime movers in this field has been Turnbull [101], who has drawn attention to the necessity for suppressing crystallization. This requires a kinetic resistance either to nucleation or to growth. Recent work [102] has focused on the resistance to nucleation of some structures in the supercooled-liquid regime between the melting point, $T_{m}$, and $T_{g}$. Turnbull points out that many glasses exhibit a high resistance to nucleation, indicative of a necessary reconstruction of the short-range order in this process (and also strong evidence against microcrystallite models for the structure of glasses). In many covalently bonded glasses, the activation energy for growth is approximately equal to the primary bond energy. One glass, $\mathrm{B}_{2} \mathrm{O}_{3}$, may be anomalous because of the unusual chemistry of boron. A modern view of resistance to nucleation in many systems would involve reconstructions from icosahedral short-range order to crystalline close-packed structures. Actually, this was first suggested over 30 years ago [56]! See Section III for a more detailed discussion of progress in this area.

The free-volume model has been refined recently. Cohen and Grest [103] applied percolation theory to calculate the communal entropy, the entropy difference between a freely flowing and a confined system of atoms. With this, they were able to analyze the thermodynamics of the glass transition. One weakness of their result is the prediction of a first-order transition, in disagreement with experiment. Chow [104] was able to derive equations of state, non-equilibrium equations, and relaxation functions by analyzing the situation in which a distribution of free volumes with different creation energies collapses. In contrast, Ibar [105] has pioneered a kinetic approach based on the assumption that a non-equilibrium state can be achieved under non-isothermal conditions if the free energy remains at its equilibrium value at the corresponding temperature. He finds it necessary to add a term to the free energy that has no obvious physical interpretation, but he can then correlate a great deal of data on polymeric systems.

\section{DYNAMIC SIMULATIONS}

\section{Dynamic phenomena}

In the previous Section, we discussed the continuous nature of the glass transition, and pointed out that a glass can be regarded as a liquid of extremely high viscosity, in which crystallization has been bypassed via rapid cooling, preventing the thermodynamically most stable crystalline configurations from being attained. The resulting glassy structures form thermodynamically metastable states, i.e., local free-energy minima stabilized by the large potential barriers separating them from the lower-lying crystalline absolute minimum and possible crystalline local minima for the same composition. A very informative and readable description of the physical phenomena involved, has been provided by Stillinger and Weber [106]. These authors show how the classification of potential minima (i.e., of mechanically stable molecular packings), offers a unifying principle for understanding properties in the solid state. They show that this approach permits identification of an inherent structure in liquids that is normally obscured by thermal motions. Melting and freezing are shown to occur through characteristic sequences of molecular packings, with a defect-softening phenomenon underlying the fact that they are thermodynamically first order phase transitions. Glass transitions and associated relaxation behavior are then explained by the topological distribution of feasible transitions between contiguous potential minima.

\section{Types of dynamic simulations}

Computer simulations of such dynamic phenomena, are extremely valuable for understanding what sequences of events take place, as well as for checking the validity of microscopic models, of theoretical assumptions and of empirical approximations. The two major types of dynamic simulations are the "Molecular Dynamics" and the "Monte Carlo" methods. In the present subsection, we will briefly summarize these two methods, combining the descriptions given by Zallen [2] and by Ziman [59]. The reader interested in more details should refer to these two excellent books. Ziman [59] also provides the historical background, and gives references to the pioneering contributions of workers such as Alder, Wainwright, Rahman, Verlet, Stillinger and Metropolis.

Consider, first, a collection of "hard-sphere molecules", described by perfectly rigid, non-interpenetrating balls of uniform size. The physical system which most closely approaches this description is an ensemble of closed-shell inert gas atoms such as $\mathrm{He}$, $\mathrm{Ne}$, $\mathrm{Ar}, \mathrm{Kr}$ and $\mathrm{Xe}$. In this idealization, the intermolecular (or pair) potential energy function 
is:

$V(r)=0$ for $r>D$, and

$V(r)=\infty$ for $r<D$.

In these equations, $D$ is the hard-sphere diameter, $\mathrm{I}$ is the separation between the centers of mass of the two "molecules", and the infinite term provides for the infinitely steep hard-sphere repulsive potential at short distances.

This hard-sphere pair potential function is obviously a gross approximation to the correct general form of $V(r)$, which is illustrated in Fig. 19. The ideal situation would be to have the exact form of $V(r)$ available from an accurate quantum mechanical calculation; however, it usually is not available. Mathematically convenient and reasonably realistic approximate general forms must therefore be sought. One such widely used form is the "Lennard-Jones 6-12 Potential":

$$
V(r)=4 \varepsilon\left[\left(-\frac{\sigma}{r}-\right)^{12}-\left(-\frac{\sigma}{r}-\right)^{6}\right],
$$

which was previously cited in Section III in the context of Frank's work [56] on icosahedral order. In this equation, the first term provides a steep repulsive interaction at short distances. The second term provides a long range van der waals attraction falling off as $1 / r^{6}$. The characteristic length and energy scales for the $V(r)$ describing a particular system, are selected by setting the dimensional quantities $\sigma$ and $\varepsilon$, respectively, to appropriate values.

The Molecular Dynamics method consists of solving the classical equations of motion and thus following the individual trajectories of several hundred molecules interacting via the chosen form of $V(r)$, given a set of initial positions and velocities, and making full allowance for the mutual interactions and collisions of these molecules. It has the advantage of simulating the motion of individual molecules, and therefore yielding useful detailed information about diffusion, correlations of velocities, correlations of the motion of neighboring molecules, etc.

In Monte Carlo simulations, a sequence of configurations is constructed by successive random displacements deriving from a random number generator. The name "Monte Carlo" is related to the similarity between this procedure and the roulette wheel used in the world's gambling capital. The simulation of the actual physical system of interest is assured by the use of the canonical ensemble, in which each configuration $\left\{\vec{r}_{j}\right\}$ appearing in the sequence, appears with a probability proportional to the Boltzmann factor,

$$
\exp \left[-V\left\{\vec{r}_{i}\right\} / k T\right],
$$

where $V\left\{\vec{r}_{i}\right\}$ is the total potential energy of the configuration, obtained as a sum of the pairwise interactions $\{V(r)\}$ between the molecules.

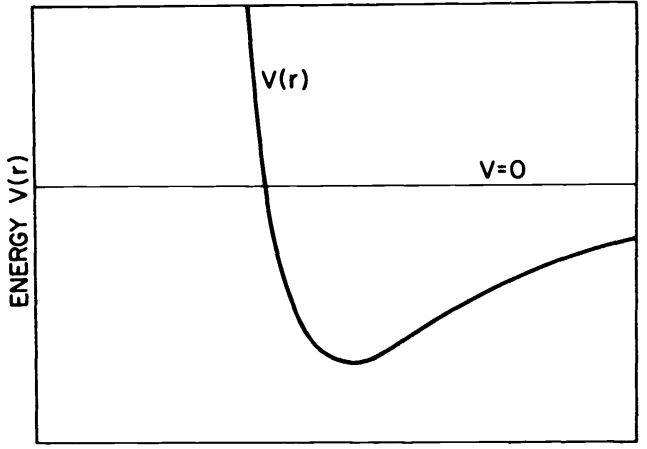

Fig. 19. General form for the interaction energy of a pair of molecules [2].

INTERMOLECULAR SEPARATION r

Unlike the Molecular Dynamics method, the particle trajectories obtained via Monte Carlo calculations do not necessarily obey the equations of motion along the path; therefore, time-evolved trajectories cannot be obtained. The advantages of the Monte Carlo method are that, for a given amount of computer time, one can deal with more particles, and perform a larger number of "moves".

Both methods can be used for the calculation of reasonable values for equilibrium thermodynamic properties, by averaging over the configurations obtained. Molecular Dynamics calculations correspond to constructing an ergodic path through a microcanonical ensemble in the phase space of the model assembly, while Monte Carlo calculations correspond to computing averages over the canonical configurational ensemble. 


\section{Some recent applications}

A detailed discussion of the vast amount of literature on the applications of dynamic simulations to physical systems is outside the scope of this review. We will instead briefly review some of the most interesting recent results, in order to give the flavor of this field, which is likely to gain even more importance with the advent of more powerful computers, as discussed in Section I.

Soules [107] has provided an extensive review of recent Molecular Dynamics and Monte Carlo calculations of glass structure and of diffusion in glasses. He has pointed out that, in general, the structures obtained closely resemble continuous random networks [5]. For example: (1) Silica glass has a cage-like structure resembling a randomly distorted high-cristobalite. (2) In simulated sodium silicate glasses, $\mathrm{Na}$ ions create non-bridging oxygen atoms and occupy interstitial sites with no well-defined coordination but a most probable coordination number of five. (3) Vitreous $\mathrm{B}_{2} \mathrm{O}_{3}$ contains planar regular triangles linked at apices to form a random network of ribbons and sheets. The Molecular Dynamics structure of $a-B_{2} O_{3}$ does not contain boroxyl rings. (4) In sodium borosilicate glasses, the trigonal-to-tetrahedral conversion of $\mathrm{B}$ with the addition of $\mathrm{Na}$, agrees with the NMR results of Yun and Bray [108]. (5) In sodium aluminosilicate glass simulations, aluminum atoms always prefer four-fold coordination, regardless of the concentration of $\mathrm{Na}_{2} \mathrm{O}$.

Soules [107] noted that all of the simulated glasses undergo a kinetic glass transition as they are cooled. Glass formation occurs when the system reaches a temperature at which the network-forming cations and anions cease to diffuse on the time scale of the Molecular Dynamics runs. "Computer experiments" can be meaningfully performed on the thermodynamic and kinetic properties of these dynamically simulated network glasses and liquids, especially at very high temperatures where the atoms are freely diffusing on the time scale of the "experiment", and at low temperatures where the system is frozen in the atomic configuration of a glass. Reasonable agreement is obtained with experimentally determined values of the properties.

Another interesting recent application of Molecular Dynamics simulations has been to the investigation of the normal modes of an amorphous solid, to study the onset of localization of modes in a Lennard-Jones-type frozen fluid, which is a true interacting many-body system [109]. This work has then been extended to the calculation of longitudinal and transverse excitations in a monoatomic, close-packed glass formed by particles interacting via a Lennard-Jones pair potential [110]. The dispersion relations obtained for the longitudinal modes look very similar to the results for polycrystalline materials, with the longitudinal dispersion curve showing a remarkably deep minimum at the wave vector where the structure factor has a peak.

Grabow and Andersen [111] have used the Molecular Dynamics method to generate model structures of amorphous metal alloys such as $\mathrm{Fe}_{80} \mathrm{P}_{20}, \mathrm{Ni}_{80} \mathrm{P}_{20}, \mathrm{Cu}_{33} \mathrm{Zr}_{67}$ and $\mathrm{Cu}_{57} \mathrm{Zr}_{43}$. They have compared their results with the results obtained by two other methods, namely, the energy minimization of dense random clusters of atoms and the energy minimization of dense random periodic structures. They indicate that the energy minimization of dense random clusters can give structures very different from those of the other two methods even when exactly the same interatomic potentials are used in the calculations, and that it should not be trusted to generate structures to be compared with experiment. They show that Molecular Dynamics is the only one of the three methods that provides a valid technique for studying both the structure of amorphous metals and structural relaxation in these materials.

Barnett et al. [112] have studied the glass transition, structure, and dynamics of a $\mathrm{Ca} 6 \mathrm{Mg}_{33}$ metalic glass via a new Molecular Dynamics approach incorporating the density dependence of the potentials. They obtain results which are in agreement with neutron scattering data. They find that the accessible configurational energy of the glass possesses several nearly degenerate potential minima, in agreement with the general physical picture presented by Stillinger and Weber [106].

Laakkonen and Nieminen [113] have used Molecular Dynamics to simulate the rapid quenching of a liquid sample to obtain an amorphous state. The two sample systems they considered were a collection of $1372 \mathrm{Ne}$ atoms interacting with a Lennard-Jones pair potential, and an $\mathrm{Fe}_{85} \mathrm{~B}_{15}$ mixture of 1204 atoms interacting with a Morse-type potential. They obtain reasonable radial distribution functions and distributions of bond angle cosines at a temperature of $4.2 \mathrm{~K}$.

These examples should serve to illustrate the wide range of physical systems and important problems which can be addressed via dynamic computer simulations, and the increasingly significant role such simulations are certain to continue to play in the future. Further discussion of the application of dynamic simulations will be provided in Sections $X I$ and XII. 


\section{ELASTIC AND VIBRATIONAL PROPERTIES}

\section{Relation to local structure}

In Section II, we mentioned some of the IR and Raman spectroscopic experiments $[4,24,38]$ used to probe the structures of non-crystalline solids. The relationship between the vibrational properties of an amorphous solid and its local atomic structure has been reviewed by Lucovsky [24] in the contexts of oxide and chalcogenide glasses ( $\mathrm{such}^{\mathrm{as}} \mathrm{a}-\mathrm{GeO}_{2}$, a-As $\mathrm{O}_{3}, a-\mathrm{As}_{2} \mathrm{~S}_{3}$ and $\mathrm{a}-\mathrm{GeSe}_{2}$ ), and of amorphous silicon-based alloys. The two major classes of approaches to the theory of the vibrational properties of amorphous solids are: ( 1 ) calculations based on finite clusters either containing a small number of atoms (generally less than 10), or a large number of atoms (generally more than a few hundred), in either case equivalent to calculating the vibrations of a large molecule; and (2) calculations based on an infinite aperiodic structure with the same coordination number at each site and with no closed loops, the Bethe Lattice, which is an artificial but useful model.

Calculations on the small clusters can be carried out with a variety of techniques, including the familiar techniques of ab initio and semi-empirical quantum chemistry. On the other hand, much simpler computational approaches, which make use of the fact that the vibrational properties are largely determined by local structural features, are needed for the large clusters containing hundreds of atoms. A common example of the use of such a large cluster is in the calculation of the vibrational properties of a CRN model large enough to adequately represent the bulk amorphous solid. The simpler computational approaches then utilized usually make use of rotationally invariant interatomic potentials with contributions from bond stretching and bond bending. Their physical meaning is therefore very similar, in the context of modeling solids, to the physical meaning of the Molecular Mechanics technique which also uses a "classical" central force model and is very familiar to chemists who compute the structures and properties of large (but finite) organic molecules and biological macromolecules.

The Bethe Lattice can easily be defined axiomatically, and used to facilitate the theoretical solution of many important physical problems, even though it cannot be realized as a physical system in a space of finite dimensionality since the prohibition against closed loops causes an endlessly increasing density as the structure is extended. An illustration of Bethe Lattices of coordination number 3 [2] and 4 [59] is provided in Fig. 20. In order to overcome the divergence problems encountered in the values calculated for physical observables with the full infinite Bethe Lattice which "overfills" space with an infinite density, "Cluster Bethe Lattice" calculations are often performed by arbitrarily truncating the full Bethe Lattice into a cluster with a finite number of atoms, often 20 to 25. Once the Bethe Lattice has been truncated into a finite cluster, the rest of the calculation is clearly no different from a calculation on a finite cluster whose atomic positions have been determined or assigned by any other method.

For the oxide and chalcogenide glasses, the Bethe Lattice method can be used to calculate local vibrational densities of states, the IR dielectric constant, and the polarized and depolarized Raman response functions [24, 114]. The glasses of interest fall into two classes: (1) those displaying strong, sharp polarized features in their Raman spectra, indicative of intermediate range order, usually due to small rings of bonded atoms (examples of this class include a-As $\mathrm{O}_{3}, a-\mathrm{GeS}_{2}$ and $\mathrm{a}-\mathrm{GeSe}_{2}$ ); and (2) those that display only broad features in their Raman spectra, indicative of the absence of small ring configurations in the intermediate range order (for example, a-GeO $2, a-\mathrm{SiO}_{2}, a-\mathrm{As}_{2} \mathrm{~S}_{3}$ and $\mathrm{a}-\mathrm{As}_{2} \mathrm{Se}_{3}$ ).

(a)

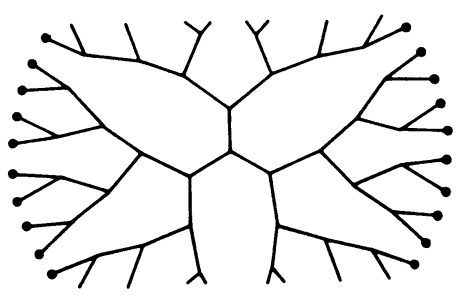

(b)

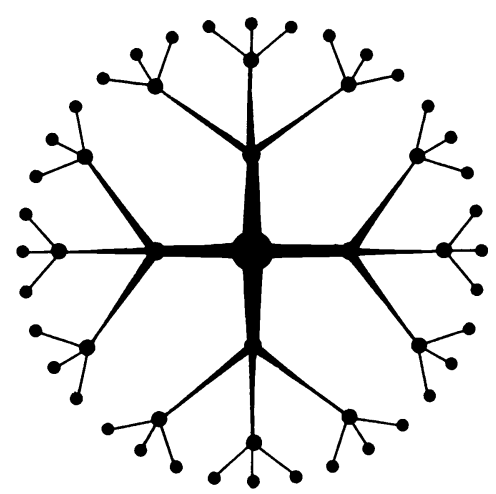

Fig. 20. Bethe Lattice, or "Cayley Tree", of (a) coordination number 3 [2., and (b) coordination number 4 [59]. In this type of lattice, note that the coordination number around each atom is held constant, but no closed rings are allowed. 
For the amorphous silicon-based alloys, most of the vibrational modes that have been identified via IR and/or Raman spectroscopy can again be understood in terms of the local bonding of the various atomic species present in the alloy. It is this strong dependence on local bonding geometry, or on rings of atoms as in several of the oxide and chalcogenide glasses, that forms the basis and the justification for the theoretical techniques that have been used as a framework for examining the vibrational properties of these materials.

Knowledge of the vibrational properties can also provide useful information about bonding in other types of materials, such as the transition metal/metalloid alloys. For example, Lustig et al. [115] reported inelastic pulsed neutron scattering experiments on amorphous Fe-P and Ni-B alloys. Their phonon density of states spectra provide information about the interatomic interactions in these alloys. The results indicate a somewhat stronger $\mathrm{Ni}-\mathrm{Ni}$ than Fe-Fe metallic interaction, as well as a stronger covalent interaction between $\mathrm{Fe}$ and $\mathrm{P}$ than between $\mathrm{Ni}$ and $\mathrm{B}$, due to increased $\mathrm{p}-\mathrm{d}$ hybridization in the Fe-P pairs.

\section{Relation to crystalline spectra}

It has been pointed out [116] that, despite the loss of long range periodicity, the properties of the elementary excitations in a glass, as described both by the vibrational density of states and by the dispersion relations for the propagation of excitations, are remarkably similar to the properties of the same excitations in the crystal of the same composition. This leads to an explanation for the origin of the low-energy vibrational modes in amorphous metallic alloys. As will be shown later, these low-energy vibrational modes are very important in understanding the structures of many types of amorphous materials.

St. Peters et al. [117] performed calculations of the vibrational properties for a model of a substitutionally disordered crystal and used the results to estimate the phonon density of states. In this model, a large cell ("supercell") of 16 or 54 atoms representing the mixed crystal $\mathrm{K}_{29} \mathrm{Rb}_{71}$ is repeated on a simple cubic lattice using periodic boundary conditions. Nearest and next nearest neighbor interactions are included. It was suggested that one could model a glass by this method as a periodically reproduced amorphous cell.

Such calculations, using periodic boundary conditions and large repeating "unit cells" of atoms, are common in the computation of the vibrational and electronic properties of non-crystalline solids. While it has often been objected that they merely describe the properties of a crystalline structure with a large number of atoms in its unit cell, they have proved to be quite useful when their results are interpreted properly. Very often, the choice is between such methods utilizing periodic boundary conditions, and alternative methods where the interpretation of the results is marred by the artifacts introduced in overcoming surface effects.

\section{Network rigidity}

Network rigidity is a very important concept in understanding the elastic and vibrational properties of non-crystalline solids, as well as in understanding their behavior under diverse stimuli such as the application of heat, light, or an electric field.

We have already encountered two examples of the role of network rigidity in Section $v$, in the subsection on glassy alloys. One of these is the difference between threshold switching and memory switching alloys [86], the current-voltage (I-V) characteristics of which are both shown in Fig. 2l. In the threshold switch, a minimum holding voltage $\left(V_{H}\right)$ must be applied to sustain the highly conductive ON state. On the other hand, in the memory switch, the excitation causing the $O F F \rightarrow O N$ transition results in an amorphous $\rightarrow$ crystalline phase
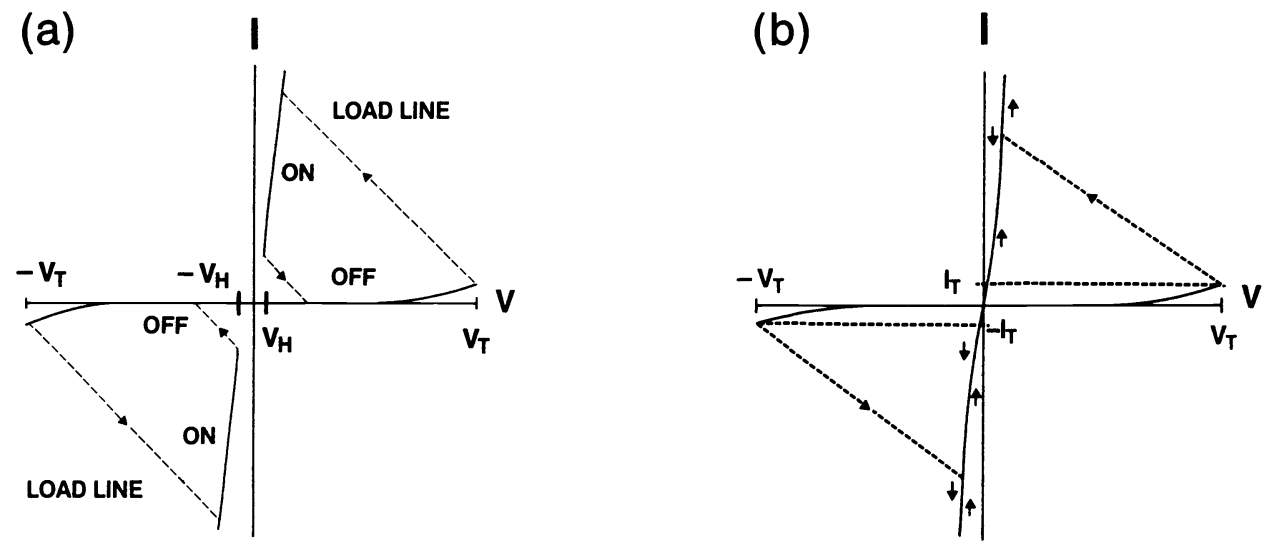

Fig. 21. Current-Voltage (I-V) characteristics for: (a) a typical threshold switch; (b) a typical memory switch. 
transition or some other more subtle structural change, often preceded by a phase separation, so that the material remains in the nonvolatile oN state even after the motivating potential is turned off. To return to the OFF state, a current pulse with a sharp trailing edge must be applied, in order to dissolve the crystallites and rapidly quench the melt in the environment which acts as a heat sink.

The average connectivity of an alloy turns out to be a crucial factor in determining whether it exhibits threshold or memory switching [86]. The differences between threshold and memory alloys have been explained in terms of the relative rigidities of the networks formed by different percentages of divalent, trivalent and tetravalent alloying elements [118-120]. To summarize, memory switches have lower energy barriers retarding amorphous-crystalline phase transitions due to weaker bonds and a more flexible network containing large concentrations of divalent elements, thus allowing the setting and resetting of the $\mathrm{ON}$ and OFF states. On the other hand, the more strongly bonded and three-dimensionally rigid threshold switches can only undergo electronic transitions which are automatically reversed when the applied voltage inducing the transition is turned off.

The other example of the importance of network rigidity, discussed in Section $V$, is the "T $-E_{g}-\bar{C}$ Correlation" of deNeufville and Rockstad [87]. A chain structure would have $\bar{C}=2$, and various chains would be held together in the solid by van der waals interactions and by intertwining. These chains can glide past each other like snakes when the system is heated, melting the solid by overcoming the relatively weak van der Waals forces without the necessity of breaking covalent bonds. This explains the $(\bar{C}-2)$ factor in the expression for $\mathrm{T}_{g}$, which is then essentially independent of bond energies for $\overline{\mathrm{C}}=2$. On the other hand, for $\overline{\mathrm{C}}>2$, $\mathrm{T}_{\mathrm{g}}$ depends linearly both on $(\overline{\mathrm{C}}-2)$, which indicates the number of cross-links that have to be broken to obtain the freely gliding chains, and on $E_{04}$, which is an index of the energy that must be supplied to break these weakest bonds. Thus, the final form

$$
T_{g} \approx T_{g}^{0}+\beta(\bar{C}-2) E_{04}
$$

can be understood in a simple way.

\section{The topological method}

A quantitative criterion for predicting which types of materials are likely to be good glass formers has been proposed by Phillips [22, 121], who used a topological viewpoint in which connectivity is measured by the average coordination number $\bar{c}$. He evaluated the number of constraints per atom that need to be introduced in order to have fixed bond lengths and fixed bond angles in a covalently bonded alloy, and showed that these can all be satisfied simultaneously in three dimensions only if $\overline{\mathrm{C}} \leq 2.4$. One implication of this result is that alloys such as $\mathrm{a}-\mathrm{As}_{2} \mathrm{Se}_{3}$, which have $\overline{\mathrm{C}}=2.4$, have the ideal network rigidity to optimize both the bond lengths and the bond angles. They should thus be excellent glass formers, i.e., yield amorphous structures after quenching from the melt at a relatively modest rate. In contrast, a material such as a-Se, with $\bar{C}=2$, will be "under-constrained", with a significant amount of freedom remaining after fixing all the bond lengths and bond angles. It is therefore possible to achieve some amount of dihedral angle ordering. This is the origin of the intermediate range order observed in a-Se [38, 122].

At the other extreme from the "under-constrained" a-Se, are the "over - constrained" materials such as a-Si and a-GaAs, both of which have $\bar{C}=4$. Such materials possess a great deal of local strains. In a-Si, much of this strain is relieved by the $\pm 10^{\circ}$ spread of bond angles from the optimum $109.5^{\circ}$ value for the tetrahedral angle. Nevertheless, because of the extent of the over-constrained nature of the network, some defect centers such as stretched bonds and under-coordinated atoms are also inevitably introduced into a-Si films during preparation, resulting in a material with large defect concentrations. The major reason why a-Si:H [90] and a-Si:H:F [42] alloys are much better semiconductors than pure $\mathrm{a}-\mathrm{Si}$ is that the monovalent alloying elements $H$ and $\mathrm{F}$ reduce the value of $\bar{c}$, relieving a considerable amount of strain and greatly reducing the defect concentration.

\section{Percolation}

Percolation theory has been rapidly gaining popularity as a technique for the analysis of disordered systems. Percolation refers to the propagation of random interconnections through a network. When the available fraction of sites, or of bonds connecting these sites, exceeds a critical "percolation threshold", it becomes possible to find a continuous pathway going through the network by only traveling through the available sites or available bonds. Although the results of percolation theory ordinarily refer to the mathematical limit of an infinitely extended network, they are applicable to solids since even a tiny 
micromolar sample of a pure element contains an enormous number $\left(\sim 6 \times 10^{17}\right)$ of atoms. It should, however, be kept in mind that the physical atoms and bonds which make up a solid have very complex interactions, and are not usually "available" or "unavailable" to the same degree of absoluteness as in a mathematical percolation model. The percolation thresholds to be derived from such models should therefore be viewed as approximate centers of a range of possible cutoff values, rather than as absolutely fixed and precise cutoff values, for the behavior of the physical system that they are expected to model.

A detailed discussion of percolation theory is outside the scope of this review, but the interested reader can refer to the books by Zallen [2] and by Ziman [59]. Here, we will merely summarize some of the recent work related to the application of the ideas of percolation theory to the elastic properties of covalent glasses.

Bicerano and Ovshinsky $[88,123]$ proposed that a percolation threshold value $\overrightarrow{\mathrm{C}}^{\mathrm{P}}$ might exist for the average coordination number $\bar{C}$, below which a switching material [86] is soft enough to undergo the structural phase transition that occurs in memory switching, and above which it is not. They suggested that $2.50<\bar{C}^{P}<2.85$, i.e., only slightly above the $\vec{C} \approx 2.4$ value derived by Phillips [22] for an ideal glass former which is nearly strain-free.

Thorpe [124] examined in detail some current ideas concerning the differences between covalent continuous random networks with high and low average coordination number. He showed how these ideas could be made rigorous by considering the number of continuous deformations (i.e., zero frequency modes) allowed within the network. In the transition from one kind of network to another, rigidity percolates through the system. This leads to a picture in which: (1) CRN's with low $\bar{C}$, such as polymeric glasses, have large floppy or spongy regions with a few rigid inclusions; (2) in CRN's with high $\bar{c}$, the rigid regions have percolated to form a rigid solid with a few floppy or spongy inclusions. He found that a mean field theory treatment of rigidity percolation gives $\vec{C}^{P}=2.4$. Covalently bonded amorphous solids with $\overline{\mathrm{C}}<2.4$ can therefore be regarded as polymeric glasses, while an amorphous solid is obtained for $\overline{\mathrm{C}}>2.4$. These ideas are illustrated in Fig. 22 [124].

I. Polymeric Glass

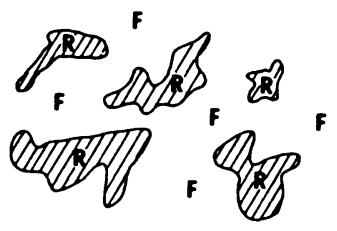

II. Amorphous Solid

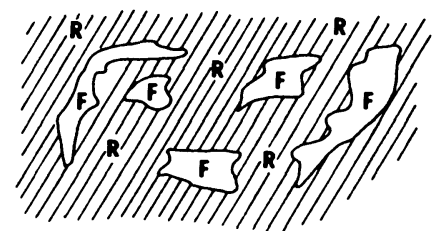

Fig. 22. Rigid (R) and Floppy $(F)$ regions in networks of Type $I$ (polymeric glass) and Type II (amorphous solid) [124].

The crucial quantity in Thorpe's analysis is the fraction of zero frequency vibrational modes. The calculation of low frequency vibrations, and the understanding of their relationships to structural features and to overall elastic properties are obviously related problems of interest $[116,125,126]$. For example, Day et al. [125] used both the Coherent Potential Approximation and numerical simulations to compute the low frequency spectrum of vibrations for the central force model, and find that the results of both calculations agree surprisingly well. Rivier and Gilchrist [126] examined a tetravalent CRN whose elasticity is described by a Keating potential, and showed the possibility of a pair of doubly degenerate ground state configurations between which the system can tunnel. (A Keating potential is a rotationally invariant form of the potential energy with contributions from bond stretching and bond bending). More work along these lines would be very valuable in improving the models which apply the ideas of network rigidity and percolation theory to non-crystalline solids.

\section{More on computational methods}

The understanding of local structure, the prediction of IR and Raman spectra, and the examination of overall elastic properties by using the techniques of percolation theory would all be greatly aided by improvements in techniques for calculating vibrational properties. A detailed description of these techniques is beyond the scope of this review; however, we will present some more of the basic physical ideas. For the reader interested in learning more about specific methods, the description given in Section 4.3 of the book by Elliott [126] is a good starting point. This reference briefly discusses issues such as the use of finite clusters with attendant surface effects as opposed to the introduction of periodic boundary conditions, and describes and compares several popular computational techniques. 
We have already seen that the computational techniques most commonly used for the calculation of the vibrational properties of amorphous solids are finite cluster calculations and Cluster Bethe Lattice calculations $[24,114]$. Since these methods are equally useful in the calculation of electronic structure and properties, they will be discussed further in Section IX.

Another versatile technique is the Recursion Method, which was originally developed for calculations of the electronic structure of disordered materials [128], and later extended to calculations of their vibrational properties [129]. In this very general, but algebraically quite complex approach, the required density of states (whether electronic or vibrational) is obtained without recourse to the direct calculation of the eigenvectors or eigenvalues of the appropriate dynamical matrix. In other words, matrix diagonalization is not needed. The technique can therefore be used for very large calculations, with less expenditure of computer time than even the familiar Extended Hückel approach which requires a matrix diagonalization step. In the Recursion Method, the basis set of states $\{|s\rangle\}$ is generated from some starting state $|i\rangle$ by successive applications of the appropriate dynamical matrix, via an algorithm that ensures the mutual orthogonality of all the states in the set $\{|s\rangle\}$. The method is best applied in a local site representation, where the initial function $|i\rangle$ is a site orbital selected by judicious consideration of the short range order, and new neighbors are introduced at each successive stage of the calculation. The technique gets its name from the recursion relations which arise from its application.

Another technique which enables the calculation of smooth spectral functions without requiring the diagonalization of a large matrix, is the Equation-of-Motion Method [130], which produces results quite similar to those produced by the Recursion Method, in spite of the seemingly quite different algorithm used. In the Equation-of-Motion Method, the equation of motion describing the lattice vibrations is integrated forward in time ( $t$ ), from $t=0$ to $t=T$, adopting reasonable values for the initial displacements and assuming that the velocities vanish at $t=0$.

A third technique which enables the calculation of the density of vibrational states without diagonalizing a large matrix, is the Negative Eigenvalue Method [131, 132]. This method uses a theorem which allows the efficient computation of the distribution of eigenvalues of a real symmetric matrix. The frequency spectra are obtained directly, while further work is required if information on a specific eigenvalue or eigenvector is needed.

Theoretical chemists can clearly make significant contributions to the development and application of computational methods for the vibrational properties of non-crystalline solids. The most popular of the computational techniques mentioned in this section, namely the finite cluster and Cluster Bethe Lattice calculations, are natural extensions to solids of methods routinely used to treat finite molecules. The Recursion Method, which appears to be less directly related to the familiar techniques of theoretical chemistry, in reality requires just as careful a consideration of the short range order in the solid (i.e., the use of chemical considerations) as the Hückel-type methods require of the bonding environment of the atoms in a molecule.

\section{ELECTRONIC STRUCTURE AND PROPERTIES OF NONMETALLIC MATERIALS}

The subject of the electronic structure of non-crystalline solids has been replete with misunderstanding, confusion, and even erroneous conclusions. This evolved from the fact that the band theory of solids developed by Bloch [133] in 1928 was really only the band theory of crystalline solids. By making use of the mathematical simplifications arising from long-range periodicity, Bloch was able to derive some general properties of the electronic states in crystals, from which wilson [134] developed a theory of electronic transport. Bloch's work followed the discovery of the periodicity of crystals by less than 20 years and the development of quantum theory by only three years. His demonstration that all the electronic states of a crystal were extended throughout the solid cleared up the "mystery of the unscattered electrons". The first theory of electronic transport proposed that the negatively charged electrons would necessarily be scattered by the positive ion cores, resulting in a mean free path of the order of an interatomic spacing; however, in many materials, the actual electronic mean free path is hundreds of interatomic spacings. After the development of Bloch-Wilson theory, it became clear that only deviations from periodicity scatter electrons in extended states, and the mystery was solved.

To this day, the standard treatments of solid-state theory are based on crystalline periodicity. To avoid handling the problem of non-crystalline solids, many texts define a solid as a periodic array of atoms, relegating non-crystalline materials to the realm of supercooled liquids. Of course, all this is nonsense. Liquids ordinarily exhibit electronic mean free paths of the order of the corresponding solids [135], so that periodicity is clearly not the answer to the mystery of the unscattered electrons. As a corollary, the Bloch-Wilson theory should be abandoned as the general approach to the electronic structure of solids. 
The modern theory of solids is actually based on chemistry rather than crystallography [16]. We can begin with a gedanken theory. In principle, a reasonable starting point is a cluster that contains at least the nearest and next-nearest neighbors of the atoms whose outer electrons are near the Fermi energy of the solid. If periodic boundary conditions are applied, the band structure can usually be calculated. The deviations from periodicity can then be handled by perturbation theory. The sharp band edges of a periodic system then disappear, and band tails result. Experimentally, these band tails presently appear to decay exponentially as the energy changes. The most important question to answer, however, is the order of magnitude of the mean free path of electrons in these bands. Very few rigorous results exist in this regard. Mott and Twose [136] showed that all electronic states in disordered one-dimensional systems are localized rather than extended, but one-dimensional systems have special properties that do not generalize (e.g., the percolation threshold is 100\%). Recent numerical calculations [137] indicate that some states in a disordered two-dimensional system are, in fact, extended. Mott [138] suggested that the bands in a real non-crystalline solid contain both localized and extended states, separated by a sharp mobility edge. Simple models based on either classical percolation [139] or quantum-mechanical tunneling [140] tend to confirm that the electronic mobility sharply increases from very small values to much larger values over a relatively narrow range of energy in the band tails of disordered systems. If these results hold up to increased rigor, the mystery of the unscattered electrons will once again appear to be resolved.

Cohen et al. [14l] pointed out that the extent of the band tails should be a measure of the disorder in the system, and noted that highly disordered solids such as multicomponent glasses should always have a finite density of states at the Fermi energy, $g\left(E_{F}\right)$. Nevertheless, if $E_{F}$ falls in a region of localized states, the material would be nonmetallic. They proposed the density of states sketched in Fig. 23 for highly disordered amorphous semiconductors. Except for the band tails, this density of states is essentially featureless, in sharp contrast to that of a crystalline solid. The structure in the gap of a crystalline semiconductor arises from the presence of so-called defects, e.g., vacancies, interstitials, and impurities forced into the wrong chemical environment by the constraints of the periodic lattice. Cohen et al. [141] proposed that such defects are not present in glasses, since in the liquid state from which they are quenched, there is no reason why a mobile atom should not be in its chemical ground state.

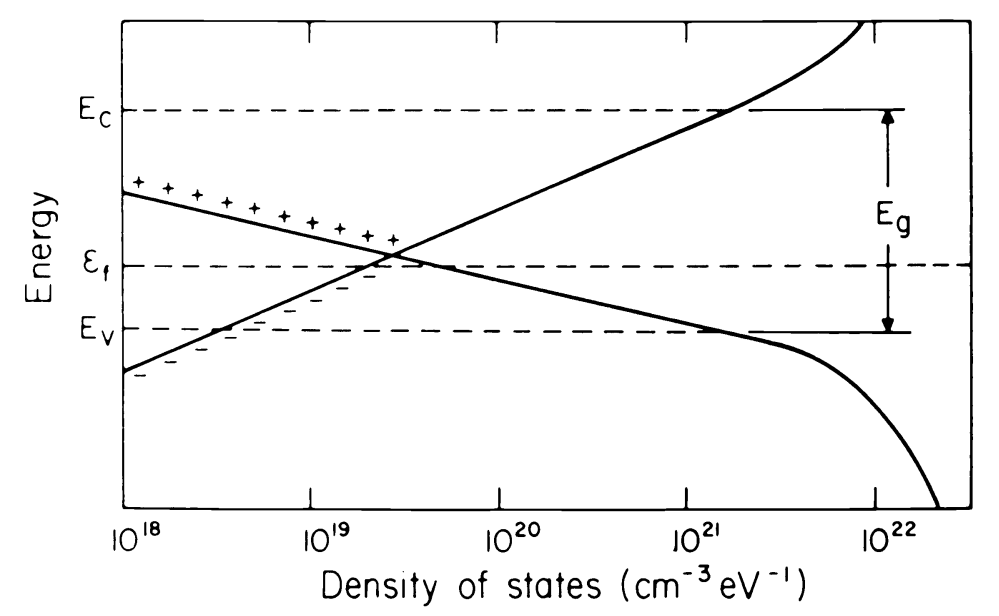

Fig. 23. CFO model for the density of states of a highly disordered amorphous semiconductor. $E_{v}$ and $E_{c}$ are the respective valence and conduction band mobility edges, $E_{g}$ is the mobility gap, and $E_{F}$ is the Fermi energy [16].

Now, many years later, the last assumption is known to be quite naive. If it were the case, substitutional doping of amorphous semiconductors would have proved impossible and the electronic properties of glasses would have been totally incomprehensible. Well-defined chemical defects can appear in glasses either to minimize the free energy at the glass transition temperature or because of internal strains upon solidification [142]. Since these defects can give rise to electronic states located near $E_{F}$, they can, in fact, control the electronic properties of amorphous semiconductors, just as in crystalline semiconductors. 
In recent years, the beginnings of a quantitative theory of amorphous solids have arisen, based on chemical considerations. The first step in this direction was the model Hamiltonian of Weaire and Thorpe [50],

$$
H=V_{1} \Sigma_{i, j \neq j^{\prime}}\left|\Phi_{i j}>\Phi_{i j}{ }^{\prime}\right|+V_{2} \sum_{i \neq i^{\prime}, j}^{\prime}\left|\Phi_{i j}>\Phi_{i} j^{\prime}\right|
$$

for tetrahedrally coordinated structures, where the atoms are denoted by the subscript $i$ and the bonds by $j$, the states $\left|\Phi_{i j}\right\rangle$ are localized $s p^{3}$ hybrid orbitals, and the matrix elements $v_{1}$ and $v_{2}$ represent the intra-atomic and interatomic interactions, respectively. This Hamiltonian makes it easy to separate the effects of quantitative disorder (i.e., bond angle variations), reflected in a spread of values of $v_{1}$ and $v_{2}$, from topological disorder (i.e., variations in ring size). Weaire and Thorpe were able to rigorously show that for constant $v_{1}$ and $v_{2}$, ranges of $v_{1} / v_{2}$ exist for which a non-zero energy gap exists in the density of states, the first demonstration that short-range order alone can produce a semiconductor.

Given the importance of short-range order and electronic correlations in localized states, simple models based on tight-binding limits have proved to be useful starting points. Pioneered by Kastner et al. [17] and Adler [143], the creation energies, one-electron energies, and effective correlation energies of defect configurations are estimated from atomic energy levels and bond energies, under the assumption that complete local atomic relaxations are possible in the amorphous network. This approach represents a rapid means of identifying the low-energy defects and estimating their effects on the electronic structure, but any quantitative conclusions must, of course, be used with extreme caution.

In order to be more precise, realistic calculations must be performed. One of the more popular recent approaches has been the empirical tight-binding technique [131], in which the matrix elements and overlap integrals are treated as adjustable parameters to be fitted to known experimental results. This method has been applied to study defect configurations in amorphous Se [144] and Si:H [145, 146].

Ab initio tight-binding calculations have also been carried out recently, often using effective Hamiltonians for simplicity [147]. Another variation, called the orthogonalized linear combination of atomic orbitals (OLCAO) method, makes use of expansions of the atomic orbitals in terms of Gaussians, with the potential constructed from a superposition of all the atoms in a small cluster, with the Slater local exchange approximation [148]. This method has been applied to study dopant defect states in a-Si:H [149].

Pseudopotential techniques [150], in which the real potential is replaced by a much smoother effective potential that yields high-accuracy results for the outer electronic states but not the core, have proved to be very useful in non-crystalline solids [151]. Since these can easily be made self-consistent, they often provide more accurate results than non-self-consistent tight-binding methods. A fairly recent application of the pseudopotential technique by Hafner [152] showed the quantitative basis for stability of metallic glasses.

Perhaps the most useful method thus far has made use of modern quantum chemistry. These include the GVB technique [94-96], discussed in Section $V$, the self-consistent-field-X $X_{\alpha}$-scattered wave (SCF-X $\left.\alpha-S W\right)$ approach [153], and the unrestricted Hartree-Fock (UHF) approximation [154], all of which have already proved valuable in the analysis of non-crystalline solids.

In addition to the particular technique, the structural model used can be critical. These can involve finite clusters, making use of the (hopefully) known short-range order, infinite clusters such as the Bethe Lattice discussed in Section VIII, supercell models, which make use of large unit cells and periodic boundary conditions [15], and effective crystal models, including the virtual crystal approximation [155] and the coherent potential approximation [156].

Messmer [157] recently discussed some of the more sophisticated calculation methods in detail and identified the major need for improving on mean-field theories, especially when localized states are important. He suggested that the GVB technique, together with the perfect-pairing approximation [158], in which all bonds are saturated, is a good starting point for the consideration of electronic-correlation effects. This approach has been applied to defects in $S i$ with great success. Zygmunt et al. [159] used the SCF-X $X_{\alpha}-S W$ technique to study defects in a-Si:H and found that aromatic-type structures are capable of stabilizing positively and negatively charged defect centers in $\mathrm{Si}-\mathrm{H}$ alloys. The presence of such centers would explain puzzling photostructural effects in these alloys in a natural way. Bernholc [160] applied the self-consistent Green's function technique [161] to a defect cluster consisting of a crystalline $S i$ vacancy with the potential dangling bonds on three of the four surrounding atoms saturated with hydrogen atoms. He obtained a positive effective correlation energy for the si dangling bond, in conflict with similar calculations 
recently carried out by Bar-Yam and Joannopoulos [162]. The sign of this energy is still one of the major unresolved problems in the study of a-Si:H [163].

Hayes and Beeby [164] suggested a new multiple-scattering approach to estimate the density of states of disordered solids. Although few calculations have actually been carried out within their scheme, the technique has the advantage of a great degree of flexibility. In a virtually orthogonal approach, Blaisdell et al. [165] have developed a computer program for the ab initio self-consistent calculations of molecular orbitals in polymeric and molecular crystals and have applied it to a linear chain of nine $\mathrm{H}_{2}$ molecules.

As an example of the current state-of-the-art, we consider the technologically important material, a-Si:H. Experimentally, pure a-Si is a poor semiconductor, with $E_{F}$ pinned by a large density of localized states in the gap. As hydrogen is introduced during the growth process, the energy gap increases and the density of states in the gap decreases. The starting point in an understanding of the electronic structure of $\mathrm{a}-\mathrm{Si}: \mathrm{H}$ is the realization that a CRN with an average coordination number of 4 is very overconstrained (recall the discussion in Section II). Consequently, a-Si is always characterized by a large concentration of strain-induced defects, especially dangling bonds. The incorporation of hydrogen lowers the average coordination number and relieves a large part of the overall strain. In addition, the $\mathrm{Si}-\mathrm{H}$ bond strength is about $40 \%$ greater than the strength of the $\mathrm{Si}-\mathrm{Si}$ single-bond. Since $\mathrm{H}$ is more electronegative than Si, tight-binding calculations indicate that the $\mathrm{Si}-\mathrm{H}$ bonding states are deep in the a-Si:H valence band, while the $\mathrm{Si}-\mathrm{H}$ anti-bonding orbitals are not too far from the conduction-band mobility edge. Sketches of the tight-binding estimates [166] for the configurations $\mathrm{Si}_{5}, \mathrm{Si}_{4} \mathrm{H}$, and the dangling bond $\mathrm{Si}_{4}$ are shown in Fig. 24. Note that even in these crude estimates, it appears that the band gap of a-Si:H should increase with hydrogen concentration, by shifting states from near the top of the valence band to considerably lower energy.

(a)

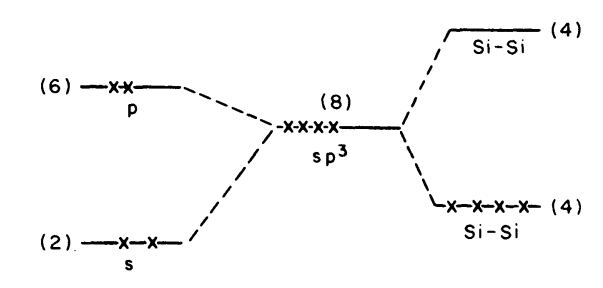

(b)

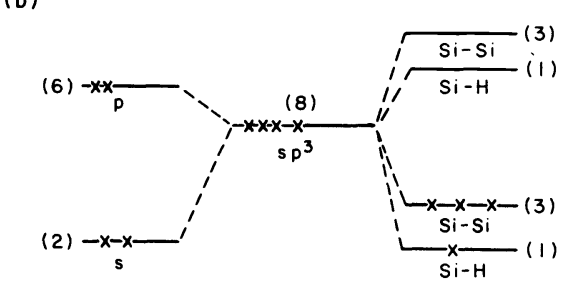

(c)

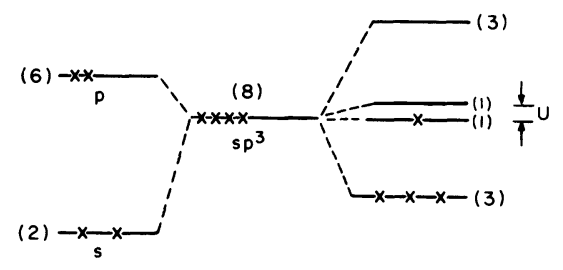

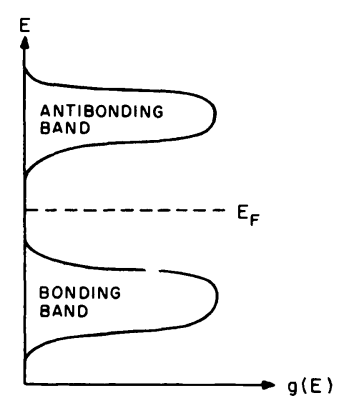
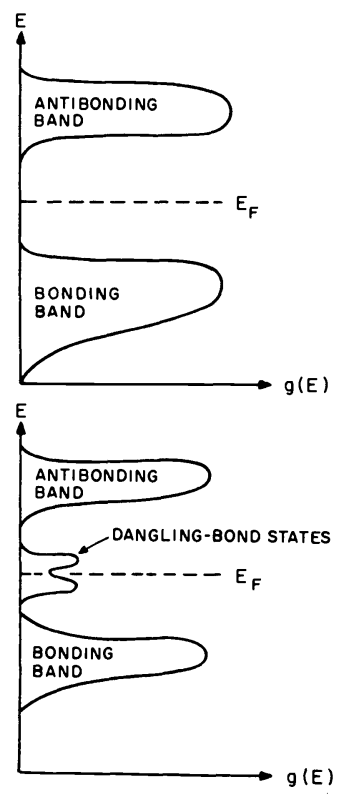

Fig. 24. Tight-binding approach to the band structure of $\mathrm{a}-\mathrm{Si}: \mathrm{H}:$ (a) A central Si atom tetrahedrally coordinated by form neighboring Si atoms; the eight $\mathrm{sp}^{3}$ hybridized orbitals are split into four bonding and four anti-bonding orbitals, which respectively spread into the valence and conduction bands of the solid. (b) A central Si atom tetrahedrally coordinated by three neighboring $\mathrm{Si}$ atoms and one $\mathrm{H}$ atom; the $\mathrm{Si}-\mathrm{H}$ bonding orbital falls deep within the valence band, while the $\mathrm{Si}-\mathrm{H}$ anti-bonding orbital lies somewhat above the lower edge of the conduction band. (c) A central Si atom surrounded by only three neighboring Si atoms; the fourth electron on the central atom occupies a non-bonding orbital (a dangling bond), separated from its unoccupied partner by the correlation energy, $V$ [166]. 
The results of more accurate SCF-X $\alpha-S W$ calculations [153] are shown in Fig. 25 . These results confirm the increase in band gap via recession of the valence band edge as its hydrogen concentration increases, and shows several hydrogen-induced peaks in the density of states $(A-D)$. Cluster Bethe Lattice calculations [145] are presented in Fig. 26 for hydrogenated surfaces of $\mathrm{C}-\mathrm{Si}$ and $\mathrm{a}-\mathrm{Si}$ and a model for a-Si:H and compares the results to experimental photoemission data [167]. It is clear that such calculations allow an understanding of the origin of the hydrogen-related structure in the data $(C, D, E)$. OLCAO results [168] are shown in Fig. 27. Although the details of the band structure differ from the other calculations, the gap and the hydrogen-induced structure are consistent.

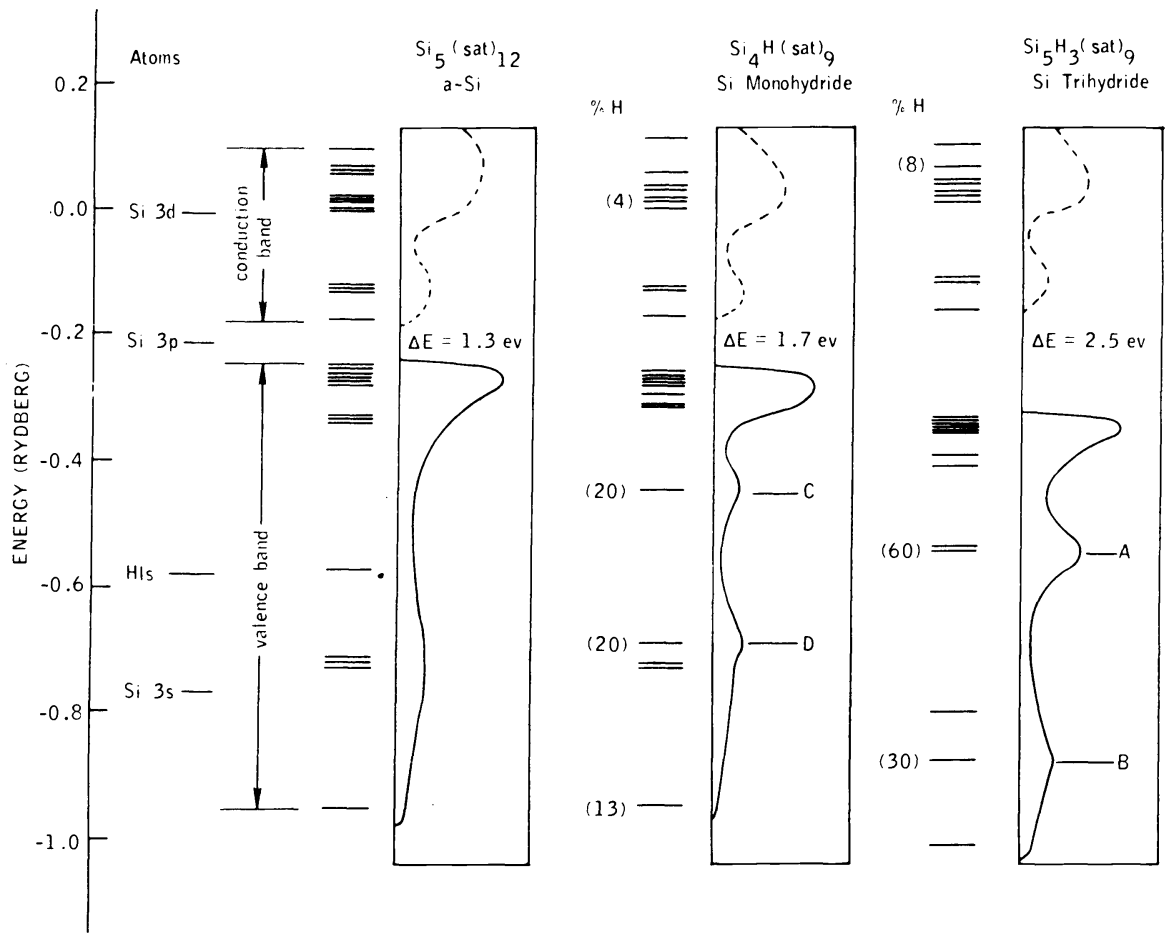

Fig. 25. SCF-X $\alpha-S W$ molecular-orbital calculations for several atomic clusters expected to be important in a-Si:H. The density-of-states curves are broadened according to a program appropriate for comparison with photoemission experiments [153].

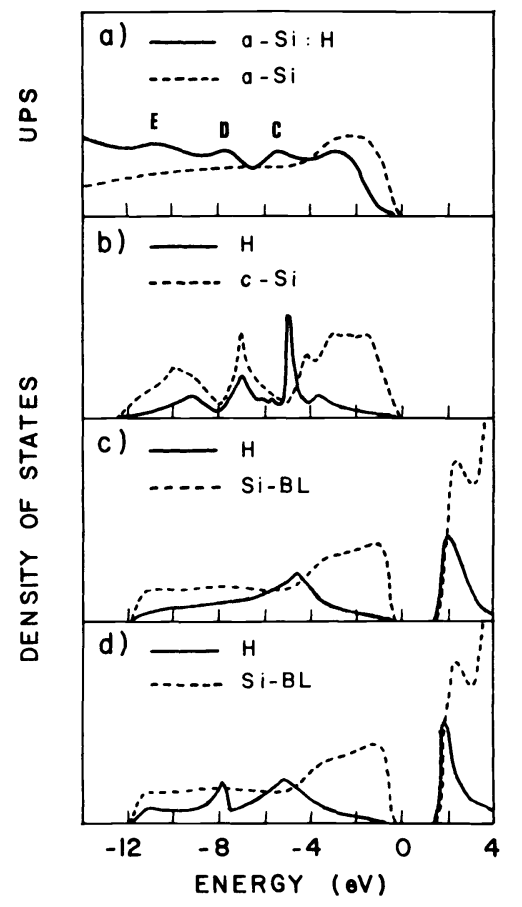

$\mathrm{Si} \quad \mathrm{O} \mathrm{H}$

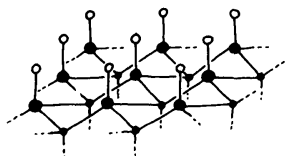

$\mathrm{H}$ monolayer on Si (III) surface

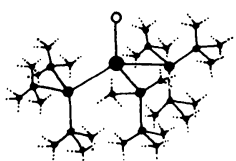

Isolated $\mathrm{H}$ in $\mathrm{Si} \mathrm{BL}$ bulk

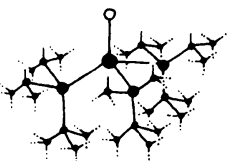

Isolated $\mathrm{H}$ on $\mathrm{Si}$ BL surface
Fig. 26. Cluster Bethe Lattice calculations for $\mathrm{C}-\mathrm{Si}$, a-Si, and $\mathrm{a}-\mathrm{Si}: \mathrm{H}$.

(a) Photoemission results;

(b) density-of-states (dashed curve) and local density on $H$ atoms (solid curve) for a hydrogenated $\mathrm{C}-\mathrm{Si}$ surface;

(c) same as part (b), but for an isolated $H$ embedded in a Si Bethe Lattice;

(d) same as part (b), but for an isolated $H$ on a Si Bethe Lattice surface [145]. 


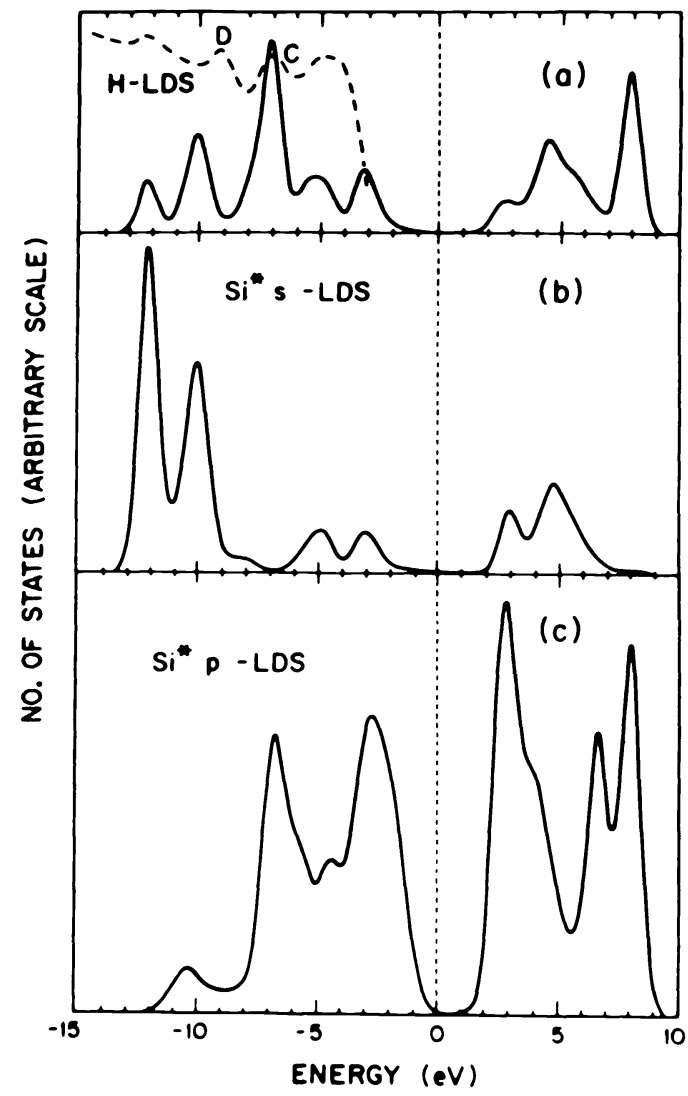

Fig. 27. Local density-of-states for an isolated $H$ atom embedded in a $S i C R N$, as calculated by the OLCAO technique [168]. The dashed line shows the photoemission results.

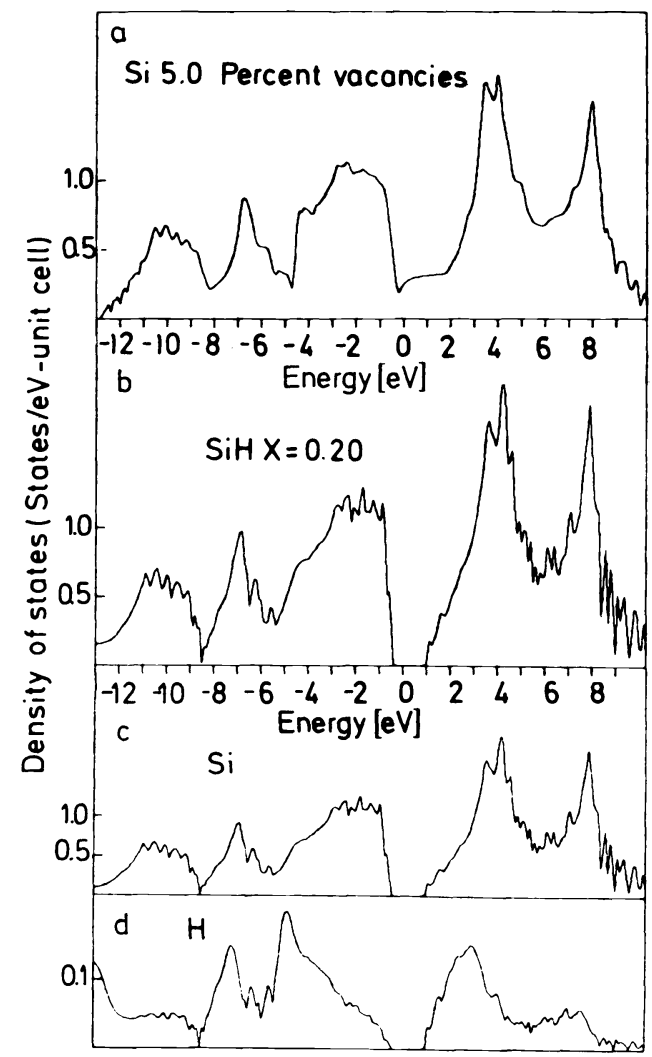

Fig. 28. (a) Total density-of-states for C-Si containing $5 \%$ vacancies, as calculated by the CPA method. (b) Same as part (a), but with the vacancies each saturated by four $H$ atoms. (c) Local density-of-states of the model of part (b) on Si. (d) Local density-of-states of the model of part (b) on H [156].

Finally, the CPA results [156] of Fig. 28 show clearly how the defect states are snowplowed out of the gap by hydrogenation, as expected.

In summary, we have come a long way since people questioned whether amorphous semiconductors were theoretically possible [169] or whether disordered materials even have a band structure. Some realistic calculations have been carried out and many new and promising techniques have been developed. Nevertheless, there are a multitude of unsolved problems that cry out for more accurate theoretical calculations to resolve [163]. The electronic structure of non-crystalline solids is certain to be an active area of research for many years to come.

\section{AMORPHOUS METALS AND ALLOYS}

Metallic glasses are technologically exciting because of their remarkable mechanical and magnetic properties. Many of them are both extremely strong and ductile, as well as being virtually non-corrosive. In addition, they can be magnetically soft (i.e., easily magnetized) while mechanically hard, so they have found applications as transformer cores and magnetic disks, among many others. The mechanical strength arises from the lack of mobile dislocations, the origin of fracture in crystalline metals and alloys. We note this is one more example of an advantage of the amorphous compared to the crystalline state. Another is the integrity of surface bonding relative to that of cleaved crystals, believed to be the origin of the low corrosion rates in amorphous solids.

There are many classes of amorphous metals. We have already mentioned the transition metal/metalloid (T-M) glasses in Section II, typically consisting of $80 \%$ from one or more late transition metal (e.g., Pd, Ni, Fe) and $20 \%$ from one of the metalloids (e.g., P, Si, B, $\mathrm{Sb})$. Another class (T-R glasses) is composed of alloys of late transition metals (e.g., 
$\mathrm{Ni}, \mathrm{Co}, \mathrm{Fe}$ ) with rare-earth atoms (e.g., Gd, La). There are also alloys of early (e.g., Nb, $\mathrm{Zn}$ ) and late transition metals (T-T glasses) and the alkaline-earth alloys, (AE glasses) which contain $\mathrm{Ca}, \mathrm{Mg}, \mathrm{Zn}$, or $\mathrm{Cd}$ combined with one or more of a large variety of other metals.

In the absence of directional covalent bonds, the question of glass-forming ability is more subtle in metallic glasses than in amorphous semiconductors, for which the continuous random network model is appropriate. In order to achieve the dense random packing structure believed applicable to a glass, other criteria have been suggested. Clearly, a deep eutectic composition helps glass forming by allowing for less-rapid cooling rates in order to reach $T_{g}$ from $T_{m}$ without crystallization, and this is borne out by experiment. Nagel and Tauc [170] suggested that glass forming is further aided by tailoring the composition so that $E_{F}$ lies near a minimum in the electronic density of states, so that small relaxations can lead to local stabilization of somewhat distorted structures; however, experimental [171] and theoretical [172] confirmation of this idea are thus far dubious at best, and much remains to be done before this area can be considered to be basically understood.

Recent work on glassy metals has tended to concentrate on deviations from dense random packing. We have already mentioned Gaskell's analysis [30] of the short and intermediate range order in T-M glasses, in which a 9-atom polyhedron centered on the metalloid is a major structural unit. The philosophy behind the dense packing of moderate-sized low-energy clusters is that the existence of more than one (but not too many) can act as a suppressant to crystallization. Some support for the general idea of dense random packing of structural units 4.5-6̊ in size comes from a recent understanding of the first sharp diffraction peak in the structure factor of a large number of diverse glasses [173]. Other work has involved computer simulations of DRP models with two different atomic radii and the introduction of atomic relaxations via Lennard-Jones or Keating potentials. Saw and Faber [174] have investigated the DRP model of a binary alloy in which either the atomic size ratio or the composition can vary. They found only small effects arising from short-range order after allowing for atomic relaxations with a Keating potential. Saw and Schwartz [175] applied the DRP model to a T-T glass, $\mathrm{Ni}_{35} \mathrm{Zr}_{65}$, and found essentially no short-range order unless different strengths for the $\mathrm{Ni}-\mathrm{Ni}, \mathrm{Zr}-\mathrm{Zr}$, and $\mathrm{Ni}-\mathrm{Zr}$ bonds were explicitly introduced. Using a computer simulation, Suzuki and Egami [176] were able to show that DRP structures are unstable against shear deformations, at least for $T=0$, consistent with some experimental data.

Electronic density-of-states calculations for glassy metals have not been performed with any degree of frequency. Kelly and Bullett [172] investigated a T-M glass, $\mathrm{Pd}_{80} \mathrm{Si}_{20}$, using clusters of about 200 atoms and a Green's function approach. Frota-Pessoa [177] and Jaswal [178] have both investigated $\mathrm{Ni}-\mathrm{Zr}$ glasses, the former using an empirical method, and the latter a self-consistent cluster approach with periodic boundary conditions and the recursion technique (see section VIII). For metallic systems, the calculated density of states can thus far be tested only by the results of photoemission experiments, which are not ordinarily sufficiently sensitive to make firm conclusions about the accuracy of the theoretical approach. In any event, more work in this area is essential.

\section{SILICA GLASSES AND RELATED ALLOYS}

Historically, fused silica $\left(\mathrm{a}-\mathrm{SiO}_{2}\right)$ and its related alloys have received a great deal of attention for thousands of years, since window glass is one member of the class. The increasing technological importance of ceramics, composites, and optical fibers is certain to ensure continued interest in these materials. It is now generally (but not universally) believed that a-SiO 2 forms a classic CRN (an example of which is the model of Bell and Dean [10]), with each Si atom surrounded by four 0 atoms with tetrahedral bond angles $\left(109.5^{\circ}\right)$, and each 0 atom surrounded by two $s i$ atoms with a spread in bond angles $\left(150^{\circ} \pm 15^{\circ}\right)$. When the CRN is modified to allow for atomic relaxations, agreement between theory and experiment becomes quite good [179]. Even Molecular Dynamics approaches have given good results for the structure of silica glasses [107,180] with the tetrahedral structure being predicted despite use of a purely ionic potential [180]. There has also been a great deal of effort expended on the study of defects in a- $\mathrm{SiO}_{2}$ [181], and microscopic models have been studied theoretically by tight-binding approaches [182] as well as by more sophisticated methods $[183,184]$. In Section VIII, we also discussed some of the work on the vibrational properties of these glasses $[24,114]$.

Studies of vibrational densities of states from CRN models have recently been reported. When only short range interactions with atomic relaxations are included [185] in a modified Keating potential, the resulting structure factor shows very good agreement with elastic neutron scattering experiments, while the vibrational density of states also shows fairly good agreement with the experiments. The fact that the double maximum observed at high frequencies of the spectrum is also found in the simulation using only short range forces [185] is encouraging. Alternatively, calculations have also been reported where, for the 
first time, long range coulomb effects were included [186] in a network with periodic boundary conditions. These calculations show that optical excitations with wavelengths much longer than the nearest neighbor separation can propagate in amorphous media, and that a longitudinal-transverse optical mode splitting is observed as in crystalline materials.

An analytical model has been built for the distribution of dihedral angles in $A x_{2}-$ type tetrahedral glasses, and its predictions compared with available experimental data [187]. The comparison is favorable for $\mathrm{a}_{-} \mathrm{SiO}_{2}$, but becomes less impressive for glasses such as $\mathrm{GeSe}_{2}$, which have much smaller intertetrahedral angles and more regular rings than $\mathrm{a}_{-} \mathrm{SiO}_{2}$. Considering the basic simplicity of the model, whose only assumptions are a local order with tetravalent $A$ and divalent $X$, and a simple type of non-bonded steric hindrance which does not include any information concerning the existence or effect of completed rings of bonds, these results are quite encouraging. Since predicting and understanding the distribution of dihedral angles in a covalently bonded non-crystalline solid can provide valuable insights on the intermediate range order (see, for example, Sections II and III, and the discussion on amorphous selenium [38]), further work along these lines can be very useful.

Another example of promising work on intermediate range order in $\mathrm{a}_{-} \mathrm{SiO}_{2}$ is the examination, by Tadros et al. [188], of ring statistics in very large computer-generated models with up to 30,000 atoms, including constraints imposed by chemical bonding on the dihedral angle distribution. These authors find that ring formation is dictated primarily by the bond angle centered at the twofold coordinated atomic site, thus explaining the absence of strong spectroscopic features [24] in the oxides of $\mathrm{Si}$ and $\mathrm{Ge}$. The calculations also suggest (not too surprisingly in our view!) that small ring formation is more probable at smaller bond angles.

The Molecular Dynamics simulation of 0choa and Simmons [189] examining the response of ${\mathrm{a}-\mathrm{SiO}_{2}}_{2}$ to longitudinal strain, is an example of the use of dynamic simulations to predict the bulk properties of a solid. For example, the effect of various atomic motions on the brittle strength, and the effect of internal cracks on fracture, are among properties which can be studied.

High resolution solid state ${ }^{29} \mathrm{Si}$ NMR studies of the local environment of $\mathrm{Si}$ in an $\mathrm{SiO}_{2}-\mathrm{Al}_{2} \mathrm{O}_{3}$ glass ceramic [190] using magic angle spinning, is an example of state-of-the-art experimental work of the type that could be very useful in studying short and long range order in these complex materials. A valuable theoretical contribution to this field would be to attempt to systematize the understanding of solid state NMR results, so that the local environment of a $\mathrm{Si}$ atom can be inferred reliably from a properly taken solid state ${ }^{29}$ NMR spectrum, just as the local environment of a hydrogen atom in an organic molecule can be inferred from the proton NMR spectrum of that molecule.

Finally, it is interesting to note that according to IR, $x$-ray photoelectron spectroscopy, and Auger electron spectroscopy experiments, suboxides of silicon $\left(\mathrm{a}-\mathrm{SiO}_{\mathrm{x}}, \mathrm{x}<2\right)$ produced by plasma enhanced chemical vapor deposition are also describable by a CRN model involving tetrahedral building blocks [191]. since there are not enough oxygen atoms to obtain a fully chemically ordered CRN (see Sections II and III), each central Si atom in a tetrahedral building block contains four near neighbors, one or more of which might be other Si atoms.

\section{LIQUIDS}

\section{Relations between liquids and glasses}

We have already encountered many of the fundamental structural, thermodynamic and kinetic considerations which relate liquids and glasses. For example, in Section VI, we discussed the continuous nature of the glass transition, and pointed out that a glass can be regarded as a liquid of extremely high viscosity, in which crystallization has been bypassed via rapid cooling, thus preventing the thermodynamically most stable crystalline configurations from being attained. The resulting glassy structures were seen to be thermodynamically metastable states, i.e., local free-energy minima, stabilized by the large kinetic barriers separating them from the lower-lying crystalline absolute minimum and possible other crystalline local minima for the same composition. In Section VII, we discussed the dynamic simulation methods which can be used to model these rapid quenching phenomena, and gave several examples of recent applications of these techniques. In Section III, we discussed liquid-to-icosahedral phase transitions, pointed out how the possibility of an icosahedral condensed phase with long range orientational order was computationally predicted on this basis, and went on to mention the relationship between the structure factor of the liquid phase and the properties of the orientationally ordered phase.

In the present section, we carry the discussion of the relationship between liquids and non-crystalline solids a little further, and then describe some of the most exciting work going on at the frontiers of this field. 


\section{Mechanisms of phase separations}

Most of the standard structural models treat non-crystalline solids as homogeneous materials. This type of approach, in addition to its important limitations at the microscopic atomic scale as discussed in section II, also neglects the presence of structural inhomogeneities on a larger scale. One common type of structural inhomogeneity in glassy alloys is compositional phase separation caused by the immiscibility of components of the melt which is rapidly cooled to form the glass. This type of phase separation occurs when the Gibbs free energy of the phase-separated mixture in the liquid is lower than that of the single homogeneous phase. A simple example of this situation is a binary system $A B$, which can exist in the liquid either as a single phase or as a phase-separated mixture, depending on which alternative minimizes the Gibbs free energy. A nice description of the phenomena involved appears in Section 3.6 of the book by Elliott [126]. To summarize, there are two distinct mechanisms which can lead to phase separation. These differ in the dependence of free energy on composition. If the compositional fluctuations must be greater than a certain size to be stable and lead to phase separation, the phase separation is said to occur by "nucleation and growth". Conversely, the single phase might be unstable to all fluctuations, of any size. In this case, phase separation does not require a nucleation step, but rather is diffusion-limited. A theory for the initial stages of this so-called "spinodal decomposition" process has been provided by Cahn [192], and by Cahn and Charles [193]. A specific physical process that can be described by the spinodal decomposition mechanism is the initial stages of the heat treatment at $450^{\circ} \mathrm{C}$ of a $\mathrm{B}_{2} \mathrm{O}_{3}-\mathrm{PbO}_{-}-\mathrm{Al}_{2} \mathrm{O}_{3}$ glass quenched from $1150^{\circ} \mathrm{C}[194]$.

\section{Recent work}

The study of liquids and their relation to glasses is a vast field of research, and we will only attempt to provide the fundamental underlying physical ideas without going into the details. Several recent papers [195-199], examine some of the problems at the frontiers of the study of liquids. The most promising techniques used include dynamic simulations, density functional calculations, hydrodynamic techniques in conjunction with consideration of phenomena which occur on a molecular length scale, and electronic structure calculations.

Kirkpatrick [195] attempted to explain the results of computer experiments using Molecular Dynamics, and considered anomalous mode coupling effects in dense classical liquids. He was able to explain the discrepancies between the results of conventional mode coupling theory and computer "experiments" in terms of a generalized mode coupling theory. This generalized mode coupling theory takes into account phenomena which occur on a molecular length scale where liquid structure is important, in addition to the phenomena usually treated with hydrodynamic mode coupling theories.

Wolynes [196] considered application of techniques from liquid state theory, such as free energy functionals for inhomogeneous systems, along with self-consistent phonon theories, in order to relate the stability of glasses to their underlying structure and to the intermolecular forces. He also reviewed recent work along these lines for the hard sphere glass. He recommends the use of more flexible trial densities, the development of more sophisticated density functionals, and the inclusion of nonequilibrium effects and fluctuations.

Chen et a1. [197] discussed transverse atomic correlations in supercooled liquids described in terms of the orientational ordering of the local shear stresses. They used Molecular Dynamics studies of liquid iron to show that the onset of the correlation as a function of temperature is rather sharp, and is accompanied by changes in diffusivity, viscosity, and the phonon density of states. They also suggested that the glass transition may be defined by this transition in a dynamical sense, by the elastic percolation of the correlated regions.

Alder et al. [198] have discussed the use of Quantum Monte Carlo calculations to simulate liquids. The Quantum Monte Carlo simulations are, in principle, quite similar to the classical simulations, although there are several additional serious technical difficulties caused by the use of quantum mechanical wave functions and probability densities instead of classical particles, and the corresponding replacement of the classical equations of motion by the Schrödinger equation. They have applied the Quantum Monte Carlo simulations to the calculation of the properties of the electron gas (the simplest model system for the condensed phase), of hydrogen, and of liquid helium. The study of the bulk properties of increasingly complex systems by Quantum Monte Carlo simulations is a promising research area which will progress as algorithms are improved and faster computers become available; however, it will probably take many years for these techniques to reach the level of maturity of the classical simulations and become as easily usable for the same types of very complex systems.

Finally, St. Peters and Roth [199] have presented an analysis of a muffin-tin model calculation of the electronic density of states of liquid copper. An ionic pair distribution function was used in this work to account for the fact that due to the short 
range liquid order, the ionic potential of a liquid is neither periodic nor random. Multiple scattering theory was used to handle the strong interactions between the localized d states and delocalized s states. The results of these calculations were seen to compare well with optical density of states measurements on molten $\mathrm{Cu}$.

In general, the electronic structure of liquids has been understood in terms of a nearly free electron approach [59], which appears to work because the temperature is large compared to the Debye temperature of the corresponding solid. Unfortunately, this is not the case in amorphous solids, so that a more complex analysis is required. The work reported by st. Peters and Roth [199] is an example, in the context of a liquid system, of how the metallic model can be modified to take some of the more complex effects into account.

An alternative to the metallic model is the so-called "distorted crystal" model [200], where the crystalline phase of a semiconductor is used as a starting point for the treatment of the electronic structure of the corresponding liquid semiconductor. This provides a zeroth-order approximation where a band structure with a band gap is already present. Two types of disorder can then be applied to this initial band structure. One of these is the topological disorder arising from the loss of long range order while some kind of short range order persists. The second type of disorder is due to fluctuations in atomic density and potential. There is clearly some vagueness and arbitrariness involved in the nature of the disorder to be applied.

Another very chemically oriented alternative to the metallic model is the so-called "molecular bond" model [200], where the idealized starting point consists of discrete molecules which are far enough that their electronic levels are discrete. When these discrete molecules are brought closer together, the discrete electronic energies broaden into bands which may be described by tight binding theory. The approximation that the electronic states of different bonds and non-bonding electrons are independent for the discrete molecules, is an additional simplification that can be used in the context of this model. This model has the advantages of being in accord with chemical intuition; of being equally applicable whether the bonding leads to network structures as in $\mathrm{As}_{2} \mathrm{Se}_{3}$, or to small molecules as in $\mathrm{Tl}_{2} \mathrm{Te}$; and of being easy to apply, since the semi-empirical methods of structural chemistry can be used for making estimates of the energy and electronic character of the bond orbitals, and these estimates can then be used to infer the electronic bands via tight binding calculations [200].

Research on the structure, dynamics and properties of liquids is certain to continue to contribute to a better understanding of glasses as well as to making new types of glassy materials for technological applications. Another (somewhat unrelated but equally important) reason for chemists to become more interested in liquids is the fact that a great deal of interesting chemistry, especially in biological systems, takes place in complex liquid media. The book by cutler [200] is highly recommended to the reader interested in learning more about liquids.

\section{SUPERLATTICES}

There has been a great deal of recent interest in artificial structures, in which complex multilayered geometries employing two or more different materials are fabricated. A simple example is an inversion layer [201], which consists of a narrow $(\approx 30 \AA)$ region near the surface of a doped semiconductor in which minority carriers move freely. For example, if sufficient negative charge is induced across the insulator of a metal/insulator/p-type semiconductor (MIS) structure by applying a positive potential to the metal, conduction-band states begin to become filled. Since the electric field acts like a potential well for the conduction-band electrons, quantum effects can be important. According to the Heisenberg Uncertainty Principle, confining electrons to a region of width $L$ introduces an additional kinetic energy of the order of:

$$
E=p^{2} / 2 m>h^{2} / 2 m L^{2}
$$

Confining an electron $\left(\mathrm{m} \sim 10^{-30} \mathrm{~kg}\right)$ to a distance $L \approx 30 \AA$ yields $E \sim 0.1 \mathrm{eV}$. This means that in order to fill the lowest quantum level in the well, an applied field sufficient to populate levels about $0.1 \mathrm{eV}$ above the conduction-band edge is required. Note that the quantization occurs in only one dimension, the direction of the applied field; the electrons move freely in the plane of the layer. When a current is passed within the layer and a magnetic field is applied, normal to the layer, the Lorentz force causes the electrons to move within the layer, perpendicular to the current. When they reach the surface, they induce a transverse electric field called the Hall field. Because of the quantum-well structure, however, the Hall voltage is also quantized. The discovery of this so-called quantum Hall effect led to the 1985 Nobel Prize in Physics [202].

The most novel artificial structures to date have involved periodic alternating thin layers of different materials, e.g., ABABAB... When the materials are crystalline semiconductors, the structures are called superlattices [203]. If the two materials in the ABAB... structure are semiconductors with different energy gaps, the different potentials in the two 
regions act like periodic potential wells for both electrons and holes in the direction perpendicular to the layers. This type of problem, a one-dimensional periodic potential, was first investigated over 50 years ago [204], and is called the Kronig-Penney model. The model suggests that in superlattices, quantum effects occur for both electrons and holes, the level separations becoming larger as the thickness of each layer decreases. Since the optical gap is the minimum energy necessary to excite an electron from a filled to an empty state, the gap in superlattices should increase with decreasing layer thickness.

One problem with superlattices is the mismatch in lattice constants when a crystalline layer is deposited on a different one, resulting in large concentrations of interface states which become important when many layers are present, often to the extent that they mask the bulk properties. This problem can be overcome by using alternating amorphous semiconductors [205]. In addition to the elimination of lattice mismatches, amorphous multilayer structures have the flexibility of an essentially infinite number of possible alloys for any of the layers as well as the possibility of controlled compositional gradients within the layers. Furthermore, there is the interesting theoretical problem of analyzing a fundamentally disordered system containing a periodic structure on the 10-100§ scale.

Two of the most investigated amorphous multilayered structures are a-Si:H/a-Si $1-\mathrm{x}_{\mathrm{X}}: \mathrm{H}$ and $\mathrm{a}-\mathrm{Si}: \mathrm{H}: \mathrm{P} / \mathrm{a}-\mathrm{Si}: \mathrm{H}: \mathrm{B}$ arrays. In both cases, the potential energy creates quantum wells for both electrons and holes; the band structure of the former is sketched in Fig. 29 . As suggested by the Kronig-Penney model, the quantum wells should yield an increasing semiconductor band gap as the thickness of the layers decreases. This is borne out by the experimental data [205], shown in Fig. 30. An interesting conclusion from these results is

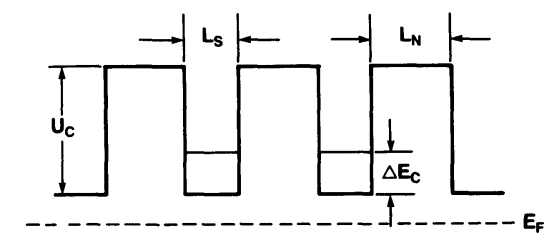

(a)
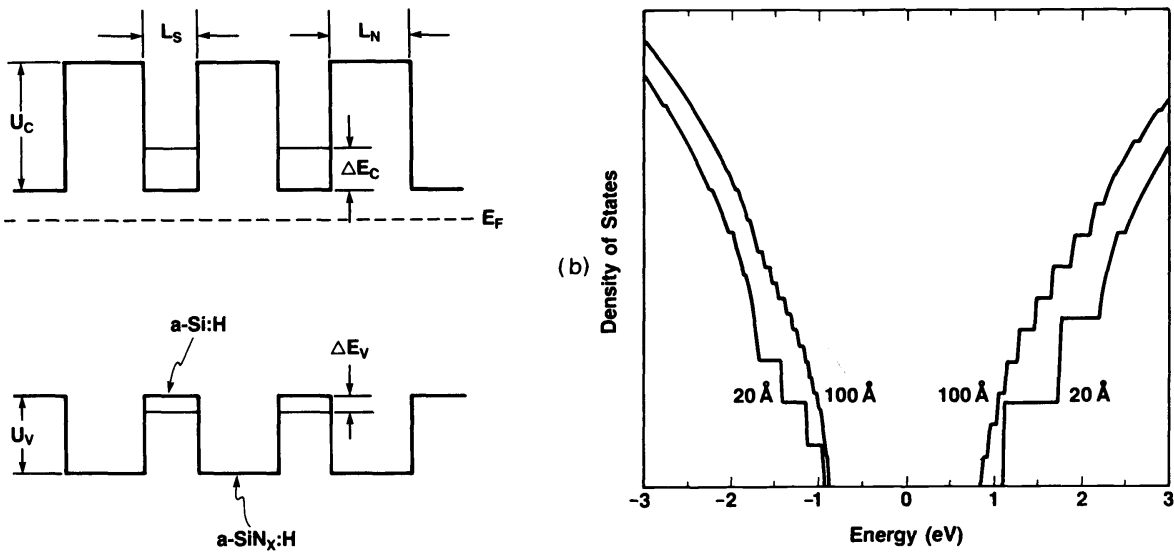

Fig. 29. (a) Potential well model for the $a-S i: H / a-S i{ }_{1-x} N_{x}: H$ superlattice; the energy levels, $\Delta E_{c}$ and $\Delta E_{v}$, for the lowest quantum states for electrons and holes, the discontinuities in conduction and valence band edges, $E_{C}$ and $E_{v}$ and the Fermi energy, $E_{F}$, are indicated. (b) Density-of-states of a free-electron gas in the presence of the potential of part (a) for a-Si:H layer thicknesses, $L_{S}$, of $20 \AA$ and $100 \AA$ [205].

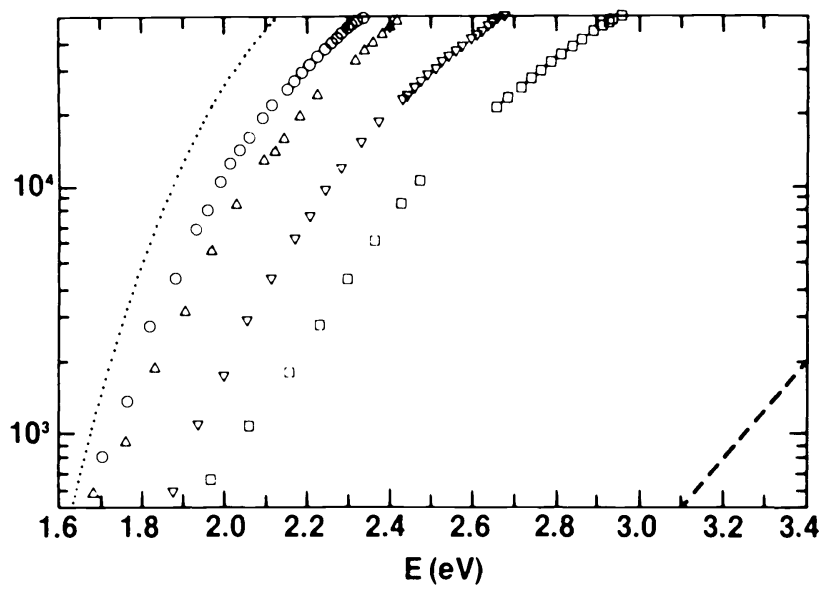

Fig. 30. Optical-absorption coefficient as a function of photon energy,

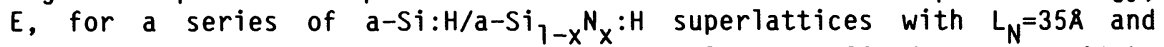
several different values of $L_{S}: L_{S}=40 \AA \quad(0), L_{S}=20 \AA \quad(\Delta), L_{S}=12 \AA(\nabla)$, $L_{S}=8 \AA(\square)$. The dotted line represents the data for bulk a-Si:H, the dashed line for bulk a-Si $1-x \mathrm{~N}_{x}: H$ [205]. 
the observation of quantum size effects in a-Si:H layers as thick as $40 \AA$, implying a wave-function coherence length greater than about 20 interatomic spacings. Tsu [206] has calculated that such a value is consistent with an electronic mean free path of about $5 \AA$. In alternating layers of a-Si:H doped $n$-type and $\mathrm{p}$-type, a persistent photoconductivity has been observed, lasting for many hours after removal of the incident light [207]. Although this could result from the reduced recombination attendant with electrons and holes being trapped in different regions of space ( $i . e .$, in the p-type and n-type layers, respectively), the effect now appears to be due to deep trapping in the interface regions [208]. Structural studies [209] have indicated that the difference between the length of the Si-Si and $\mathrm{Si}-\mathrm{N}$ bonds leads to interface distortions in $\mathrm{a}-\mathrm{Si}: \mathrm{H} / \mathrm{a}-\mathrm{Si}{ }_{1-x^{N}} \mathrm{~N}_{\mathrm{H}}$ multilayer structures.

\section{CONCLUSIONS}

Any study of a condensed collection of atoms must begin with an understanding of the structure. For a solid, which retains its shape under at least moderate shear stresses, we are aided by the conclusion that each atom is located near a local potential minimum. The empirical investigations of the structure of non-crystalline solids reviewed in this paper have, above all, focused attention on the importance of the chemical nature of the constituent atoms in the formation of such materials. Great strides have been made both experimentally and theoretically over the past few years, and the results finally appear to be converging, even if slowly [210]. Theoretical approaches have progressed far beyond the ball-and-stick models of 15 years ago, and now not only computer simulations with atomic relaxations but Monte Carlo and Molecular Dynamics techniques have produced results that portend a useful future for such calculations.

Given the structure of the material, it becomes possible to analyze both the vibrational properties (i.e., phonon modes) and the electronic behavior (i.e., electronic density of states). The former often controls the thermal properties of the solid, such as specific heat, thermal expansion, and thermal conductivity. But the electronic structure determines the response of the material to applied electric fields, visible and ultraviolet light, and a host of coupled effects such as thermoelectric, magnetoelectric, and optoelectronic behavior. The modern theory of crystalline solids has achieved its major successes from the detailed quantitative understanding of such behavior as functions of temperature and even time (under transient conditions). This is an area in which a great deal of work is still necessary in the case of non-crystalline solids.

The recent insights afforded by curved space approaches and three-dimensional projections of higher-dimensional structures has opened up new avenues for theoretical investigation. In addition, the recognition of the importance of well-defined defects in non-crystalline solids has suggested additional uses for such points of view. The fact that an infinite number of dimensions are available for modeling suggests that this area will remain fruitful for quite some time to come.

Since all solids are formed at finite temperatures and pressures, thermodynamic and kinetic considerations are vital. Difficulties in calculating such quantities as entropy and Gibbs free energy for systems with no long-range order have retarded progress, but makes these quantities no less important. Just because we are not yet smart enough to evaluate the states of local free-energy minima does not retard the atom's ability to find them rather quickly upon solidification.

A classic problem in the study of solids has been the rapid progress in experimental techniques, which often has led to the feeling that theoretical understanding will never catch up. Recent work in picosecond spectroscopy, superlattice structures, and ultra-rapid solidification, among many other developments, has intensified such feelings. But sooner or later, the theoretical insight must come or experimental progress will decelerate. The present gaps between theory and experiment stand as an exciting challenge and opportunity. The fact that closing this gap necessarily requires the cooperation between chemists, physicists, and materials scientists should only add to the appeal of working in this field.

\section{Acknowledgements}

This review article was stimulated by the International Conference on the Theory of the Structures of Non-Crystalline Solids, held at the Institute for Amorphous Studies, Bloomfield Hills, Michigan, U.S.A. We would therefore like to especially thank Stanford R. Ovshinsky, President of Energy Conversion Devices, Inc., whose support and encouragement made this Conference possible; Martin C. Steele and Brian B. Schwartz, respectively the former and present Directors of the Institute; and Ghazaleh Koefod, Executive Administrator of the Institute. We are also indebted to the International Union for Pure and Applied Chemistry for their invitation to prepare this review. In addition, we owe a great intellectual debt to our many colleagues who have helped us to understand various aspects of this vast field of research, and especially to those who participated in the Conference and its active discussion periods. Finally, we wish to thank Ella M. Norman, who patiently typed the entire manuscript. 


\section{REFERENCES}

1. Proceedings of the International Conference on the Theory of the Structures of Non-Crystalline Solids, edited by D. Adler and J. Bicerano, J. Non-Cryst. Solids, Volume 75 (North-Holland Physics Publishing, Amsterdam, 1985).

2. R. Zallen, The Physics of Amorphous Solids (John Wiley and Sons, New York, 1983).

3. Physical Properties of Amorphous Materials, edited by D. Adler, B.B. Schwartz and M.C. Steele, Institute for Amorphous Studies Series, Volume 1 (Plenum Press, New York, 1985).

4. R. Zallen, Ref. 1, p. 3

5. W.H. Zacharaisen, J. Am. Chem. Soc., 54, 3841 (1932). For extensions of the Zacharaisen schematics, see B.E. Warren, J. Am. Ceram. Soc., 24, 256 (1941) and J.F. Shackelford, J. Non-Cryst. Solids, 49,19 (1982). An excellent review of Zacharaisen's contribution, in a historical context, is provided by A.R. Cooper, Jr., J. Non-Cryst. Solids, 49, 1 (1982).

6. D.E. Polk, J. Non-Cryst. Solids, 5 , 365 (1971).

7. D.E. Polk and D.S. Boudreaux, Phys. Rev. Lett., 31,92 (1973).

8. R.J. Temkin, W. Paul and G.A.N. Connell, Adv. Phys., 22, 581 (1973).

9. P.J. Steinhardt, R. Alben and D. Weaire, J. Non-Cryst. Solids, 15, 199 (1974).

10. R.J. Bell and P. Dean, Phil. Mag., 25, 1381 (1972).

11. M. Popescu, Ref. 1, p. 477.

12. M. Popescu, Ref. 1, p. 483.

13. F. Wooten and D. Weaire, J. Non-Cryst. Solids, 64, 325 (1984); F. Wooten, K. Winer and D. Weaire, Phys. Rev. Lett., 54, 1392 (1985); F. Wooten, G.A. Fuller, K. Winer and D. Weaire, Ref. 1, P. 45; and K. Winer and F. Wooten, J. Molecular Graphics, $\underline{3}$, 76 (1985).

14.

A. Lapiccirella, N. Tomassini, K.W. Lodge and S.L. Altmann, J. Non-Cryst. Solids, 63, 301 (1984).

15. L. Guttman and C.Y. Fong, Phys. Rev. B, 26, 6756 (1982).

16. D. Adler, Ref. 3, p. 5.

17. M. Kastner, D. Adler and H. Fritzsche, Phys. Rev. Lett., 37, 1504 (1976).

18. D. Adler, Solar Cells, 2, 199 (1980).

19. A.A. Lebedev, Trans. State Optical Inst., 2, No. 10, (1921) and Rev. d'Optique Theor. et Inst., $\underline{5}, 4$ (1926).

20. P.J. Bray, Ref. 1, p. 29.

21. S.R. Ovshinsky, Ref. 3, p. 105.

22. J.C. Phillips, J. Non-Cryst. Solids, 34 (1979) 153 and J. Non-Cryst. Solids, 43, 37 (1981).

23.

P. Boolchand, Ref. 3, P. 221; J. Grothaus and P. Boolchand, J. Non-Cryst. Solids, 72, 1 (1985).

24. G. Lucovsky, Ref. 3, p. 277.

25. J.D. Bernal, in Liquids: Structure, Properties, Solid Interactions, edited by T.J. Hughel (Elsevier, Amsterdam, 1965) P. 29.

J.L. Finney, Proc. Royal Soc. (London) A, 319, 479 (1970).

J.A. Barker, M.R. Hoare and J.L. Finney, Nature, 257, 120 (1975).

C.H. Bennett, J. Appl. Phys., 43, 2727 (1972).

P.H. Gaskell, Ref. 1, p. 329.

P.J. Flory, Science, $\underline{188}, 1268$ (1975).

A.C. Wright, Ref. 1, p. 15.

R.P. Messmer and J. Wong, J. Non-Cryst. Solids, 45, 1 (1981).

A.C. Wright, G. Etherington, J.A.E. Desa, R.N. Sinclair, G.A.N. Connell and J.C. Mikkelsen, Jr., J. Non-Cryst. Solids, 49, 63 (1982).

35. A. Bienenstock, Ref. 3, p. 171 .

E.A. Stern, Ref. 3, p. 201 .

L. Cervinka, J. Komrska and J. Mikes, Ref. 1, p. 69.

G. Lucovsky and C.K. Wong, Ref. 1, p. 51 .

Webster's New World Dictionary, edited by David B. Guralnik (Simon and Schuster, New York, 1982) p. 46.

40. R.T. Morrison and R.N. Boyd, Organic Chemistry, Second Edition (Allyn and Bacon, Inc., Boston, 1971) p. 403.

41. D.E. Sands, Introduction to Crystallography (W.A. Benjamin, Inc., New York, 1969 ).

42. S.R. Ovshinsky and A. Madan, Nature, 276, 482 (1978).

43. R. Tsu, S.S. Chao, M. Izu, S.R. Ovshinsky, G.J. Jan and F.H. Pollak, J. de Physique, Colloque C4, Suppl. to No. 10, 42, C4-269 (1981).

44.

45. S.R. Ovshinsky, Revue Roumaine de Physique, 26, 893 (1981).

Liquid Semiconductors, (Edinburgh, Scotland, 1977),p. 519.

46. S.R. Ovshinsky and D. Adler, Contemp. Phys., 19, 109 (1978).

47. C. Kittel, Introduction to Solid State Physics, 5th Edition (John Wiley and Sons, New York, 1976).

48. R. Haydock, Ref. 1, p. 121 .

49. C.G. Montgomery, Ref. 1, p. 265. 
50. M.F. Thorpe and D. Weaire, Phys. Rev. B, 4, 3518 (1971) and D. Weaire and M.F. Thorpe, Phys. Rev. B, 4, 2508 (1971).

51. W.N. Lipscomb, Science, 196, 1047 (1977)and J. Less-Common Metals, 82, 1 (1981).

52. J. Bicerano, D.S. Marynick and W.N. Lipscomb, Inorg. Chem., 17, 3443 (1978).

53. J. Donohue, The Structures of the Elements, (Robert E. Krieger Publishing Company, Malabar, Florida, 1982), Chapter 5.

54. K. Katada, Japan. Jour. Appl. Phys., 5,582 (1966).

55. F.C. Frank and J.S. Kasper, Acta Crystallogr., 11,184 (1958) and Acta Crystallogr., 12, 483 (1959).

56. F.C. Frank, Proc. Roy. Soc. London Ser., 215, 43 (1952).

57. Physics Today, Vol. 35, No. 5 (May, 1982) is a special issue on liquid crystals. See especially J.D. Litster and R.J. Birgeneau, p. 26.

58. G. Graff, High Technology, 4, 54 (1984).

59. J.M. Ziman, Models of Disorder (Cambridge University Press, Cambridge, 1979).

60. R. Pindak, D.E. Moncton, S.C. Davey and J.W. Goodby, Phys. Rev. Lett., 46, 1135 (1981).

61. D. Levine and P.J. Steinhardt, Ref. 1, p. 85.

62. R. Penrose, Bull. Inst. Maths, and Its Appi., 10 (1974) 266 and M. Gardner, Sci. Am., 236, 110 (1977).

63.

64 .

65.

66.

67.

68.

69.

P.J. Steinhardt, D.R. Nelson and M. Rouchetti, Phys. Rev. Lett., 47, 1297 (1981).

P.J. Steinhardt, D.R. Nelson and M. Rouchetti, Phys. Rev. B, 28, 784 (1983)

A.D.J. Haymet, Phys. Rev. B, 27, 1725 (1983).

D. Shechtman, I.Blech, D. Gratias and J. W. Cahn, Phys. Rev. Lett., 53, 1951 (1984).

D. Levine and P.J. Steinhardt, Phys. Rev. Lett., 53, 2477 (1984).

D.R. Nelson and S. Sachdev, Acta Met., to be published, is an excellent review article. For a more detailed treatment of order, frustration, and defects in liquids and glasses, see D.R. Nelson, Phys. Rev. B, 28, 5515 (1983).

M.E. Eberhart, K.H. Johnson, D. Adler, R.C. O'Handley and M.E. McHenry, Ref. 1, p. 97.

70. C.L. Henley, Ref. 1, p. 91.

71. A.D.J. Haymet, Ref. 1, p. 79.

72 .

73.

74.

75.

H.A. Jahn and E. Teller, Proc. Roy. Soc. London A, 161, 220 (1937).

L. Pauling, Nature, 317, 512 (1985). Also see the editorial by J. Maddox on $p$.

471 , in the same issue of Nature.
J. F. Sadoc, Ref. 1, p. 103 .

76.

Metals Handbook, Eighth Edition, Volume 8, Metallography, Structures and Phase Diagrams, (American Society For Metals, 1973).

77.

78.

79 .

80 .

81 .

82.

83.

84.

85.

86.

87.

88.

89.

90 .

R. Mosseri and J.-F. Sadoc, Ref. 1, p. 115 .

T. Ninomiya, Ref. 1, p. 489.

R. Schilling and P. Reichert, Ref. 1, p. 129.

J.C. Lesjaunias and A. Ravex, J. Phys. F 13, L101 (1983).

P. Erdös and R.C. Herndon, Adv. Phys. 31, 63 (1982).

P.A. Mello and N. Kumar, Ref. 1, p. 135 .

M.A. Klenin and K. Promislow, Ref. 1, p. 141.

S.R. Ovshinsky, Ref. 1, p. 161.

S.R. Ovshinsky, Phys. Rev. Lett., 21, 1450 (1968).

J.P. deNeufville and H.K. Rockstad, in Amorphous and Liquid Semiconductors, $J$. Stuke and W. Brenig, eds., Taylor and Francis, London, (1974) 419.

J. Bicerano and S.R. Ovshinsky, Ref. 1, p. 169. A much more detailed review is provided by $J$. Bicerano and S.R. Ovshinsky, in Proc. Nobel Laureates Symposium on Applied Quantum Chemistry, D. Reidel Publishing Company, in press.

J.C. Phillips, Phys. Rev. B, 28, 7038 (1983).

W.E. Spear and P.G. LeComber, Solid State Commun., 17, 1193 (1975).

R. Grigorovici and P. Gartner, Ref. 1, p. 177.

R. Manaila, D. Macovei, R. Grigorovici and P. Pausescu, Ref. 1, p. 183.

M. Popescu, Ref. 1, p. 189.

M.H. McAdon and W.A. Goddard III, Ref. 1, p. 149.

W.A. Goddard III, T.H. Dunning, Jr., W.J. Hunt, and P.J. Hay, Acc. Chem. Res., $\underline{6}$, 368 (1973).

W.A. Goddard III and L.B. Harding, Ann. Rev. Phys. Chem., 29, 363 (1978).

J.H. Rose, J.R. Smith and J. Ferrante, Phys. Rev. B, 28, 1835 (1983).

C.D. Gelatt, Jr., A.R. Williams, and V.L. Moruzzi, Phys. Rev. B, 27, 2005 (1983).

J.H. Gibbs and E.A. DiMarzio, J. Chem. Phys., 28, 373 (1958).

M.H. Cohen and D. Turnbul1, J. Chem. Phys., 31, 1164 (1959).

D. Turnbu11, Contemp. Phys., 10. 473 (1969).

D. Turnbu11, Ref. 1, p. 197.

M.H. Cohen and G.S. Grest, Phys. Rev. B, 20, 1077 (1979) and 24, 4091 (1981).

T.S. Chow, Ref. 1, p. 209.

J.P. Ibar, Ref. 1, p. 215.

F.H. Stillinger and T.A. Weber, Science, 225, 983 (1984).

T.F. Soules, J. Non-Cryst. Solids, 49, 29 (1982). 
108.

109.

110.

111.

112.

113.

114.

115.

116.

117.

118.

119.

120.

121.

122.

123.

124.

125.

126.

127.

128.

129 .

130.

131.

132.

133.

134.

135 .

136 .

137.

138

139.

140.

141.

142.

143 .

144.

145.

146.

147.

148.

149.

150.

151.

152.

153.

154.

155.

156.

157.

158.

159.

160.

161.

162.

163.

164.

165.

Y.H. Yun and P.J. Bray, J. Non-Cryst. Solids, 27, 363 (1978).

S.R. Nage 1, A. Rahman and G.S. Crest, Phys. Rev. Lett., 47, 1665 (1981).

G.S. Grest, S.R. Nagel and A. Rahman, Phys. Rev. Lett., $\underline{49}, 1271$ (1982).

M.H. Grabow and H.C. Andersen, Ref. 1, p. 225.

R.N. Barnett, C.L. Cleveland and U. Landman, Phys. Rev. Lett., 5, 2035 (1985).

J. Laakkonen and R.M. Nieminen, Ref. 1, p. 237.

R.B. Laughlin and J.D. Joannopoulos, Phys. Rev. B, 16, 947 and 2942 (1976); J.D. Joannopoulos, J. Non-Cryst. Solids, 32, 241 (1979); and G. Lucovsky, C.K. Wong and W.B. Pollard, J. Non-Cryst. Solids, 599660,839 (1983).

N. Lustig, J.S. Lannin, D.L. Price and R. Hasegawa, Ref. 1, p. 277.

J. Hafner, Ref. 1, p. 253.

M.A. St. Peters, J.R.D. Copley and D.W. Taylor, Ref. 1, p. 271.

S.R. Ovshinsky and K. Sapru, in Amorphous and Liquid Semiconductors, edited by $J$. Stuke and W. Brenig, (Taylor and Francis, London, 1974)

p. 447.

S.R. Ovshinsky, Phys. Rev. Lett., 36, 1469 (1976).

S.R. Ovshinsky, AIP Conf. Proc., 31, 31 (1976)

J.C. Phillips, J. Non-Cryst. Solids, $35 \& 36,1157$ (1980).

T. Takahashi, K. Ohno and Y. Harada, Phys. Rev. B, 21, 3399 (1980).

J. Bicerano and S.R. Ovshinsky, J. Non-Cryst. Solids, 74, 75 (1985).

M.F. Thorpe, J. Non-Cryst. Solids, 57, 355 (1983).

A.R. Day, R. Tremblay and A.-M.S. Tremblay, Ref. 1, p. 245.

N. Rivier and H. Gilchrist, Ref. 1, p. 259.

S.R. Elliott, Physics of Amorphous Materials, (Longman, New York, 1984).

See the review by V. Heine, Solid State Phys., 35, 1 (1980).

P.E. Meek, Phil. Mag., 33, 897 (1976).

D. Beeman and R. Alben, Adv. Phys., 26, 339 (1977).

P. Dean, Rev. Mod. Phys., 44, 127 (1972).

R.J. Bel1, Rep. Progr. Phys., 35, 1315 (1972).

F. Bloch, Z. Physik, 52, 555 (1928).

A.H. Wilson, Theory of Metals, (Cambridge University Press, New York, 1936).

D. Adler, Amorphous Semiconductors (CRC Press, Cleveland, 1971).

N.F. Mott and W.D. Twose, Adv. Phys., 10, 107 (1961).

G.M. Scher and D. Adler, in Localization and Metal-Insulator Transitions, edited by H. Fritzsche and D. Adler (Plenum Press, New York, 1985) 441.

N.F. Mott, Adv. Phys., 16, 49 (1967).

M.H. Cohen, J. Non-Cryst. Solids, 4,391 (1970).

D. Adler, Solar Energy Mat., 8, 53 (1982).

M.H. Cohen, H. Fritzsche, and S.R. Ovshinsky, Phys. Rev. Lett., 22, 1065 (1969).

D. Adler, J. Solid-State Chem., 45, 40 (1982).

D. Adler, Phys. Rev. Lett., 41 , 1755 (1978) and J. Non-Cryst. Solids, 36,819 (1980).

D. Vanderbilt and J.D. Joannopoulos, Phys. Rev. B, 22, 2927 (1980).

D.C. Allan, J.D. Joannopoulos, and W.B. Pollard, Phys. Rev. B, 25, 1065 (1982) and 26,3475 (1982).

J. Robertson, Phys. Rev. B, 28, 4647, 4658, and 4666 (1983).

D.W. Bullett, Solid State Phys., 35, 129 (1980).

W.Y. Ching and C.C. Lin, Phys. Rev. B, 12, 5536 (1975).

W.Y. Ching and C.C. Lin, A.I.P. Conf. ProC., 73, 151 (1981).

M.L. Cohen and V. Heine, Solid State Phys., 24,38 (1970).

M.J. Kelly, Solid State Phys., 35, 296 (1980).

J. Hafner, in Glassy MetaTs, edited by H.J. Guntherodt and H. Beck (Springer-Verlag, New York, 1981).

K.H. Johnson, H.J. Kolari, J.P. de Neufville, and D.L. Morel, Phys. Rev. B, 21, 643 (1980).

A.C. Kenton and M.W. Ribarsky, Phys. Rev. B, 23, 2897 (1981).

K. Tanaka and R. Tsu, Phys. Rev. B, 24, 2038 (1981).

E.N. Economou and D.A. Papaconstantopoulos, Phys. Rev. B, 23, 2042 (1981).

R.P. Messmer, Ref. 1, p. 285.

F.W. Bobrowicz and W.A. Goddard, in Modern Theoretical Chemistry: Methods of Electronic Structure Theory, Vol. 3, edited by H.F. Schaefer (Plenum Press, New York, 1977) p. 79

S.A. Zygmunt, M.E. Eberhart, K.H. Johnson, and D. Adler, Ref. 1, p. 297.

J. Bernholc, Ref. 1, p. 305.

J. Bernholc, N.0. Lipari, and S.T. Pantelides, Phys. Rev. B, 21, 3545 (1980).

Y. Bar-Yam and J.D. Joannopoulos, J. Non-Cryst. Solids, $77 \& \frac{78}{78}, 99$ (1985).

D. Adler, in Physics of Disordered Materials, edited by $\frac{1}{D}$. AdTer, H. Fritzsche, and S.R. Ovshinsky (Plenum Press, New York, 1985) p. 287.

T.M. Hayes and J.L. Beeby, Ref. 1, p. 311. For the detailed implementation of this approach, see J.L. Beeby and T.M. Hayes, Phys. Rev. B, 32, 6464 (1985).

J.J. Blaisde11, W.A. Sokalski, P.C. Hariharan, and J.J. Kaufman, Ref. 1, p. 319. 
166. D. Adler, in Semiconductors and Semimetals, Vol. 21A, edited by J.I. Pankove (Academic Press, New York, 1984) p. 291.

167. D.J. Chadi, J. Vac. Sci. Technol., 16, 1290 (1979).

168. W.Y. Ching, D.J. Lam, and C.C. Lin, Phys. Rev. B, 21, 2378 (1980).

169. J.M. Ziman, J. Non-Cryst. Solids, 4,426 (1970).

170. S.R. Nagel and J. Tauc, Phys. Rev. Lett., 35, 380 (1975).

171. P. Oelhafen, E. Hansen, and H.J. Guntherodt, Solid State Commun., 35,1017 (1980).

172. M.J. Kelly and D.W. Bullett, J. Phys. C, 12, 2531 (1979).

173. S.C. Moss and D.L. Price, in Physics of Disordered Materials, edited by D. Adler, H. Fritzsche, and S.R. Ovshinsky (Plenum Press, New York, 1985)p. 77.

174.

175 .

C.K. Saw and J. Faber, Ref. 1, p. 347.

175. C.K. Saw and R.B. Schwartz, Ref. 1, p. 355.

176. Y. Suzuki and T. Egami, Ref. 1, p. 361 .

177. S. Frota-Pessoa, J. Phys. F, 15, 287 (1985).

178. S.S. Jaswa 1, Ref. 1, p. 373.

179. P.H. Gaskell and I.D. Tarrant, Phil. Mag. B, 42, 265 (1980).

180. C.A. Ange 11, P.A. Cheeseman, J.H.R. Clarke, and L.V. Woodcock, in The Structure of Non-Crystalline Materials, edited by P.H. Gaskell (Taylor and Francis, London, 1977) p. 191.

181. J. Wong and C.A. Ange 11, Glass: Structure by Spectroscopy (Dekker, New York, 1976).

182. G. Lucovsky, Phil. Mag. B, 41, 457 (1980).

183. J. Robertson, Phys. Chem. GTasses, 23, 1 (1982).

184. M. O'Keefe and G.V. Gibbs, J. Chem. Phys., 81, 876 (1984).

185. L. Guttman and S. Rahman, Ref. 1, p. 419.

186. S.W. De Leeuw and M.F. Thorpe, Ref. 1, p. 393.

187. F.L. Galeener, Ref. 1, p. 399.

188. A. Tadros, M.A. Klenin and G. Lucovsky, Ref. 1, p. 407.

189. R. Ochoa and J.H. Simmons, Ref. 1, p. 413.

190. B.C. Gerstein and A.T. Nicol, Ref. 1, p. 423.

191. G. Lucovsky, S.Y. Lin, P.D. Richard, S.S. Chao, Y. Takagi, P. Pai, J.E. Keem, and J.E. Tyler, Ref. 1, p. 429.

192. J.W. Cahn, J. Chem. Phys., 42, 93 (1965).

193. J.W. Cahn and R.H. Charles, Phys. Chem. Glasses, 6,181 (1965).

194. J. Zarzycki and F. Naudin, J. Non-Cryst. Solids, 1,215 (1969).

195. T.R. Kirkpatrick, Ref. 1, p. 437.

196. P.G. Wolynes, Ref. 1, p. 443.

197. S.-P. Chen, T. Egami and V. Vitek, Ref. 1, p. 449.

198. B.J. Alder, D.M. Ceperley and E.L. Pollock, Acc. Chem. Res., 18, 268 (1985).

199. M.A. St. Peters and L.M. Roth, Ref. 1, p. 455.

200. M. Cutler, Liquid Semiconductors (Academic Press, New York, 1977).

201. T. Ando, A.B. Fowler, and F. Stern, Rev. Mod. Phys., 54, 437 (1982).

202. K. von Klitzing, Festkorperprobleme, 21, 1 (1981).

203. L. Esaki and R. Tsu, IBM J. Res. Dev., i4, 61 (1970).

204. R. del. Kronig and W.G. Penney, Proc. Roy. Soc. (London), A130 (1931) 499.

205. B. Abeles and T. Tiedje, in Semiconductors and Semimetals, Vol. $21 \mathrm{C}$, edited by J.I. Pankove (Academic Press, New York, 1984) p. 407.

206. R. Tsu, Ref. 1, p. 463.

207. J. Kakalios and H. Fritzsche, Phys. Rev. Lett., 53, 1602 (1984).

208. S.C. Agarwal and S. Guha, Phys. Rev. B, 31, 5547 (1985).

209. N. Maley and J.S. Lannin, Ref. 1, p. 469.

210. D. Weaire, Ref. 1, p. 497. 